SLAC- -333

DE89 006019

\title{
THE APPLICATION OF LIGHT-CONE QLANTIZATION TO QUANTUM CHROMODYNAMICS IN ONE-PLUS-ONE DIMENSIONS
}

\author{
Kent. John I!ornbostel \\ Stanford Linear Accelerator Center \\ Stanford Cnixcrsity \\ Stanford. California 9.4:304
}

Decomber losis

Prepared for the Department of Energy

under contract number DE-AC03-76SF00515

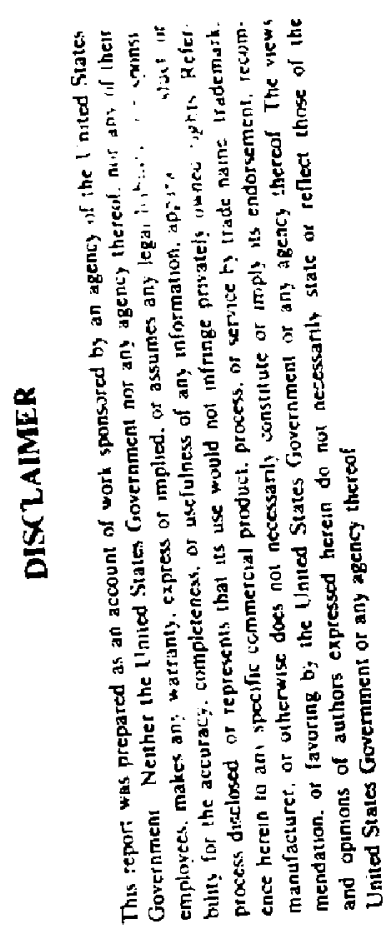

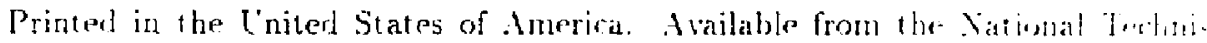

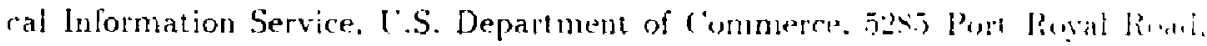
Springtield. Virginia 22161. Price: Printerl (opy A0s. Mirrofirhe 10!

\section{MASTER}




\title{
THE APPLICATIGN OF LIGHT-CONE QUANTIZATION \\ TO QUANTUM CHROMODYNAMICS \\ IN ONE-I'LUS-ONE DIMENSIONS
}

\author{
lient John Hornbostel. Ph.D. \\ Stanford Ciniversity: 1959
}

Formal and computational aspects of light cone quantization are studied by application to quantum chromodynamies (QCD) in one spatial plus one temporal dimension. This quantization scheme, which has been extensively applied to perturbative calculations, is shown to provide an incuitively appealing and numericalls tractable approach to non-perturbative computations as veil.

In the initial section, a light-cone quantization procedure is developed which incorpotates fields on the boundaries. This allows for the consistent treatnent of massless fermions and the construction of explicitly conserved momentum and charge operators.

The next section. which comprises the majority of this work, focuses on thet nunerical solution of the light-cone Schrodinger equation for bound states. The state space is constructed and the Hamiltcnian is svaluated and diagonalized by computer for arbitrary number of colors, baryon number and coupling constant sirength. As a result. the full spectrum of mesors and baryons and their associated wavefunctions are determined. These results are compared with thuse which exist from other approaches to test the reliability of the method. The progran a!su provides a preliminary test for the feasibility of, and an opportunity to deseb; approximation schemes for, an attack on three-plus-one dimensiona! QCD. 
Finally, analytic results are presented which include a discussion of integral equations for wavefunctions and their endpoint behavior. Solutions for hadronic masses and wavefuctions in the limits of both large and small quark mass are discussed. 


\section{Acknowledgements}

It is a pleasure to recognize those who have contributed their ideas and encouragement to this work, in particular, to Hans-Christian Pauli and Gary McCartor. and to acknowledge the hospitality of the Max Planck Institute for Nuclear Physics. where this work began. I would also like to thank Professors Sid Drell, Michael Peskin, and Lenny Susskind for serving on my orals commitlee, and Dick Blankenbecler for this as well as for his continuing interest in this work and his unerring advice. Thanks especially to my friends and co-laborers from the third floor night stifl, past and present, who made SLAC the place to be when there was no place else to go. Finally, I would like to express my gratitude to my advisor, Stan Brodsky, for bis freely shared time and expertise, and even more for his unparalleled combination of enthusiasm and patience. 
For Mom and Dad, Scott and Marc 


\section{Table of Contents}

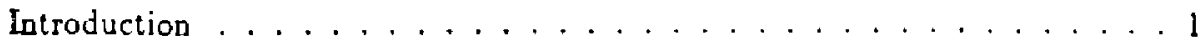

\section{Chapter 1. Quantization}

1.1 Light-Cone Quantization $\ldots \ldots \ldots \ldots$

1.2 Long Range Fields and Conserved Charges . . . . . . . . . . 5

1.3 Quantization of Free Fermions . . . . . . . . . . . .

1.4 Quantization of $S U(N)_{1+1} \ldots \ldots \ldots \ldots$

1.5 Discrete Hamiltonian, Momentum and Boost Operators . . . . . . . 29

1.6 Lorentz and Parity Properties of Warefunctions . . . . . . . . . 30

1.7 Chiral Aumaly . . . . . . . . . . . . . . . . . . 41

Chapter 2. Numerica! Results

2.1 Overview of Program $\ldots \ldots \ldots \ldots \ldots \ldots$

2.2 Spectra $\ldots \ldots \ldots \ldots \ldots \ldots \ldots \ldots \ldots \ldots$

2.3 Quark-Antiquark Valence Wavefunctions _ . . . . . . . . . G3

2.4 Structure Functions $\ldots \ldots \ldots \ldots$

2.5 Valence Structure Functions $\ldots \ldots \ldots$. . . . . . . . . . Gti

2.6 Generai Wavefunction Results . . . . . . . . . . . ci 
2.7 Higher-Fock Wavefunctions $\ldots \ldots \ldots \ldots \ldots$

2.8 Higher-Fock Wavefunctions at Weak Coupling $\ldots \ldots \ldots$

2.9 A Preliminary Look at Renormalization . . . . . . . . . . . . . 89

Chapter 3. Analytic Results

3.1 Valence Meson Integral Equations $\ldots \ldots \ldots$. . . . . . . . 91

3.2 Yalence Baryon lntegral Equations $\ldots \ldots \ldots$

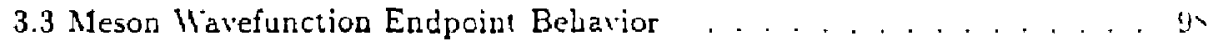

3.4 Baryon Wavefunction Endpoint Belavior . . . . . . . . . 100

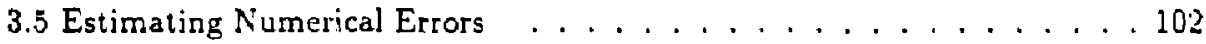

3.6 Estimate of Necessary Resolution . . . . . . . . . . . 100

$3 . i$ Higher-Fock Equations: Endpoints and Errors $\ldots \ldots \ldots \ldots$

3.8 Heary Quark Limit for Mesors . . . . . . . . . . . . . . 11:

3.9 Heavy Quark Linit for Baryons . . . . . . . . . . . . . . . . . 123

3.10 Massless Mfesons and Baryons at Strong Coupling . . . . . . . . 127

3.11 Excited Meson States at Strong Coupling . . . . . . . . . 134

3.12 Hadron Masses at Small Quark Mass . . . . . . . . . . 13 s

3.13 Comparison with Results from Bosonization , . . . . . . . . I4!

3.14 Matrix Elements for Small Quark Mass . . . . . . . . . . . 145

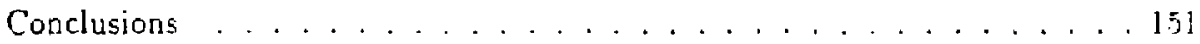


Appendix A. Matrix Elements and Color Contractions . . . . . . . . . . 15'2

Appendix B. Evaluation of Integral in Section 3.3 . . . . . . . 15i

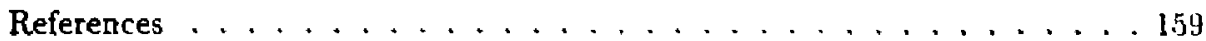




\section{List of Figures}

page

1. Surfaces Used to Define Charges. . . . . . . . . . . . . 6

2. Development of $N=3$ Spectrum kith Increasing $K_{;}$Weak Coupling. . . 4!

3. Development of Spectrum with Increasing $k$; Strong Coupling. . . . . . 50

4. Development of $N=3$ Spectrum with Coupling Constant $\lambda . \ldots$. . . . . 52

5. Lightest Meson and Baryon Mass versus Quark Mass

for $X^{*}=2,3$ and $4 . \ldots \ldots \ldots \ldots$

6. Comparison of Lightest Meson Mass for $\Lambda=2,3$ and 4 with

Lat tice Calculation for $N=2 . \ldots . \ldots . . . \ldots$. . . . . . . . . .

7. Comparison of Lightest Mleson Mlass for $N=2,3$ and 4 with

Large- $N$ Meson. . . . . . . . . . . . . . . . . . . . 59

8. Lightest Meson Mass for $S L(2)$ and $L(2)$. . . . . . . . . . . . . . . 6?

9. Wavefunctions for First Fire $N=3$ Mesons in V'alence

Approximation. ...................... 61

10. Structure Functions for the Lightest $N=3$ Mfeson and Baryon. . . . $6 \sigma^{-}$

11. Siructure Functions for the Valence and Four-Quark $N^{\prime}=3$

Meson Wavefunctions at Weak Coupling. . . . . . . . . . . . . . . 69

12. Structure Functions for the Valence and Five-Quark $\Lambda=3$

Baryon Wavelunctions at lleak Coupling. . . . . . . . . . . . 70

13. Structure Functions for the Valence and Eight-Quark $N=3$

Two-Raryon Wavelunctions at Weak Coupling. . . . . . . . . . . i 
14. Structure Functions for the Valence and Four-Quark $N=3$

Meson Wavefunctions at Strong Coupling. . . . . . . . . . . i3

15. Structure Functions for the Valence and Five-Quark $N=3$

Baryon Wavefunctions at Strong Coupling. . . . . . . . . . it

16. Structure Functions for the Valence and Eight-Quark $N=3$

Two-Baryon Wavefunctions at Strong Coupling. . . . . . . . . . 72

17. Contribution to Lightest $N=3$ Meson Structure Function

from Four-Quark Wavefunction. . . . . . . . . . . . is

18. Contribution to Lightest $N=3$ Baryon Structure Function. $\ldots \ldots$ ii

19. Contribution to Lightest Meson Structure Function

from the Four-Quark Wavefunction as a Function of $N \ldots \ldots \ldots$ is

20. Contribution to Lightest $U(N)$ Meson Structure Function

from the Four-Quark Wavefunction as a Function of $x \ldots \ldots$. . . . . . .

21. Comparison of Single Meson Four-Quark Higher-Fock Wavefunctius

with Wavefunction of a Pair of Valence Mesons. . . . . . . . . E 1

22. Dependence of $N=2$ Meson Higher-Fock W'avefunction

on Momentum $K \ldots \ldots \ldots \ldots \ldots$

23. Momentum Splitting from $q \bar{q}$ to $q \bar{q} q \bar{q}$ Fock States in Mleson. $\ldots . . . \quad$.

24. Momentum Splitting in $S U(3)$ Barrons. . . . . . . . . . in

25. Running Coupling Constant Defined by Holding Fixed

the $N=2, B=0$ Meson Mass to $M^{2} / \mathrm{m}^{2}=6$.

26. Numerical Approximation of Principal-Va'ue-Regulaced Integral. 
27. Extrapolation Fit to $S U(2)$ Lightest Meson Mass. . . . . . . . . 10s

2S. Typical Diagrams which Contribute to the

Integral Equation for $\phi_{2}(x) \ldots \ldots \ldots \ldots \ldots \ldots$

29. Terms in the Integral Equation for $\phi_{4}$ which Contribute t,o the

Leading Small-x Behavior. . . . . . . . . . . . . . 115

30. Leading Terms in Large- $N$ Limit for the $\phi_{4}$ Integral Equation. . . . . 116

31. Comparison of Lightest Meson Mass for $N=2,3$, and 4 with

Nonrelativistic Solutions. . . . . . . . . . . . . . . . . . . 123

32. Integration Contour $\ldots \ldots \ldots \ldots \ldots$ 
1. Four Quark Interactions in Hamiltonian. . . . . . . . . 32

2. Diagonal Interactions. . . . . . . . . . . . 33

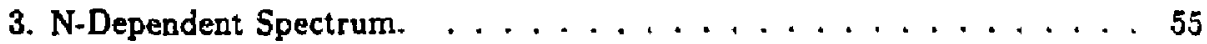

4. Ratio of First Excited State to Lightest State; $N=2, B=0 . \ldots \ldots 6$

5. Magnitude of Higher-Fock in $S U(2)$ Meson as a Function of $N . \ldots$. . . 80

6. Magnitude of Higher-Fock Component in Lightest $S L$ (2) Meson

Versus $r_{t} / g \ldots \ldots \ldots \ldots \ldots$

7. Number of States in Fock Space as a Function of the Invariant Cutoft. $\ldots \ldots \ldots \ldots \ldots$

8. Estimate of K Needed for Numerical Aecuracy as a Furction of Coupling Constant. . . . . . . . . . . 10

9. Estimate of Excited Meson Spectrun: at Strong Coupling. . . . . . . . 13;

10. Suppression of the Valence Wavefunction Due to the Presence of Higher-Fock Components. . . . . . . . . . . . . . . 143

11. Small Quark Mass (from Bosonization) and Large Quark Mass

Limits for Ratio of Meson to Baryon Mass for $N$ from 2 to $4 . \ldots$. . . .

12. Measured Ratio of Meson to Baryon Mass for $.1=3$ and $4 . \ldots$. . . . is 


\section{INTRODLCTION ${ }^{[1]}$}

Quantum Chromodynamics $(Q C D)$, a feld theory of strong interactions based on the gauge group $S U(3)$, potentially describes all of hadronic and nuclear physics in terms of quarks and gluons as fundamental degrees of freedom. The shortdistance structure of this theory, probed in processes involving large transfers of momentum, is relatively well understood. In this region, $Q C D$ is asymptotically free, and the effective interaction strength diminishes with increasing momentum. As a result, perturbative calculations become possible, and thus far have provided the best opportunities for experimental confrontation and confirmation. However. the most significant and intrinsically nonperturbative aspects of the theory. its pre. dictions for the spectrum and wavefunntions of badrors, as well as the mechanismls for confinement and jet hadronization, remain unsolved.

Lattice gauge theory in which the Feynman path integral is evaluated on a discrete spacetime grid, provides an appropriate tool for such calculations. For strong coupling. it provides an appealing description of confinentent. Numerica! results in genera] bave been consistent with experiment, if qualitative, and there is little doubt their accuracy will inprove with increasing computing fower.

Nevertheless, there is certainiy room for the development of other methods. In this work, the gene:al nonperturbative approach to field theory. "Discretized Light-Cone Quantization (DLCQ)", introduced by Pauli and Brodsky in Ref.?.? is applied to $S U(\Lambda)$ gauge theories in one space and one time $(1+1)$ dimensions as an initial step in that direcion. In addition to this numerical approach, analytic solutions in the limits of boith strong and weak coupling are presented. Throug!.ia!. 
the quarks are limited to one flavor, but several values of the number of colors, $N$. are included.

$S U\left(N^{\prime}\right)$ gauge theories restricted to $1+1$ dimensjons possess certain specia: properties which should be mentioned for the salie of orientation. Because there are no transverse directions, the gluons are not dynamical, and (in $A^{+}=0$ gaugs.) their presence is felt only by the constraint equation they leave behind. Likewise the quarks carry no spin. The fermion field mat be represented as a two-component spinor, and chirality for massless fermions identifies only the direction of motion. The coupling constant $g$ carries the dimension of mass, so that the theory is superrenormalizable. Also, for one quark havor of mass $m$, the relevant measure of coupling strength is $\mathrm{g} / \mathrm{m}$. Finally, the restriction to one spatial dimension produces confinement automatically, even for $Q E D_{1+1}$. The electric field is unable to spreat out and the energy of a non-singlet state diverges as the length of the system.

In spite of these idiosyncrasies, these models possess certain qualities $10 \mathrm{com}$. mend their study, not the least being tractability. There ar. only so many opjut. tunitjes to solve, albeit numerically, a confining field theory with arbitrary co!spling from first princjples. With solutions in hand, conceptual questions, point s of principle, or approximation schemes for $Q C D$ which do not depend on the dinen:sionality of the model may be addiessed. Also. these models provide a concreti example on whic - to test approximation schemes and numerical techaiques which may prove useful for realistic problems, and a means to check thone, such as the large- $N$ expansion, already in use. Liltimately, though. the motivation for this study is regative. If these modeis cannot be solved. there is no hope for apprys this method to $Q C D$ in $3+1$ dimensions. 


\section{QUANTIZATION}

\subsection{LIGHT-CONE QUANTIZATION}

Quantization on the light-cone is formally similar to standard canonical equal. time quantization, but wits a few technical differences which nevertheless make life much easier. ${ }^{\text {|Jl }}$ Given a (Loren:2-invariant) Lagrangian $\mathcal{L}\left(\boldsymbol{x}^{\mu}\right)$, a new variable $x^{+} \equiv x^{0}+x^{3}$ is defined to play the role of time, along with new spatial variables (in four dimensions), $x^{-} \equiv x^{0}-x^{3}$ and $x_{\perp} \equiv\left(x^{1}, x^{2}\right)$.

In these coordinates, the non-zero elements of the metric tensor are

$$
\begin{aligned}
& g^{+-}=g^{-+}=2 \\
& g_{+-}=g_{-+}=\frac{1}{2} \\
& g^{\prime \prime}=g_{1 j}=-\varepsilon^{\prime \prime} .
\end{aligned}
$$

wih $i$ and $j$ representing the (transverse) directions $I$ and 2 . (inder longitudinal Lorentz boasts (that is. in the $x^{3}$ direction) $x^{+}$and $x^{-}$transform as exp $\left( \pm \alpha \mid x^{*}\right.$. The quantization surface $x^{+}=0$ is preserved, accounting for some of the simplifier! Lorentz properties of this scheme. This is especially true in two dimensions, where only this boost is possible. Because the Hamiltonian $P^{-}$changes by only an overall scale, its eigenstates are Lorentz-invariant. Under parity transformations, however. $x^{+}$and $x^{-}$are interchanged, and while parily will be conserved. its realization wii! not be as simple.

Independent degrees of freedom are identified by the equations of mutiun. These are initialized to satisfy canonical commutation relations at $x^{*}=0$. aru' 
the creation and annibilation operators from their momentum space expansiou define the Fock space. The nomenta conjugate to $x^{-}$and $x_{\perp}, P^{+}$and $P_{\perp}$ respectively, are diagonal in this space and conserved by interactions. $P^{-}$acts as a Hamiltonian; in general it is complicated, depentent on the coupling constant, and it generates evolution in $x^{+}$. Diagonalizing it is equivalent to solving the equation. of motion.

The mass shell condition, $p^{2}=m^{2}$, for individual quanta implies that $p^{-}=$ $\left(m^{2}+p_{\perp}^{2}\right) / p^{+}$, so that positive (light-cone) energy quanta must also carry positive $p^{+}$. This seemingly innocent detail is actually a very good thing; the positivity of $p^{+}$combined with its conservation is responsible in lasge part for the simplicity of this approach. First, $x^{+}$-ordered perturbation theory becomes calculationally viable because a large class of diagrams which appear in the time-ordered analogue vanish.| These include any diagram containing a vertex in whick quanta are created out of the vacuum; since all $p^{+}$are positive, at such a vertex the total momentum cannot be conserved.

More importantly for the work described here, but by essentially the same reasoning, the perturbative vacuum is an eigenstate of the full, interacting Hamil. tonian, with eigenvalue zero. Pairs of quanta cannot be produced which conserve $p^{+}$(s) One very desirable feature of this remarkable fact is that not winl $y$ is thie ground state trivial, but also that all the quanta occurring in higher states are associated with meson and baryon wavefunctions rather than disconsected pieces of the vacuum.

Finally, it greatly simplifies the nunerical work, especially in $1+1$ dimensiulls." The system is quantized in a box of length $L$ in the $I^{-}$direction with apprupria". 
boundary conditions so that momenta are discrete and Fock space states denumerable. For the fixed total momentum $P^{+}$, the relevant dimensionless momentum will be $K^{\prime}=\frac{L}{2 \pi} P^{+}$. To see how $K$ restricts the space of states, consider $K=3$, which must be partitioned anong the quanta in each state. The only three possibilities are $(3),(2,1)$, and $(1,1,1)$. Contrast this with equal-time Fock states of definite $P^{\mathrm{I}}$. For equivalent numerical momentum, partitions will include not only those enumerated above, but also $(4,-1),(104,-101),(5,5,3,3,-1,-10)$, and so on. To keep the number of states finite, an additional cutoff in momentum must be introduced. whereas this is nol necessary in the light-cone case.

Not only does a fixed $K$ act implicity as a momentum cutof, it also severel: limits both the total number of states of definite momentum and the number of quazta in each individual state, as the example above demonstrates. $K$ serves one more role. The continuum limit $L \rightarrow \infty$ is equivalent to $K \rightarrow \infty$ as the physical momentum $P^{+}$remains fixat The size of $s^{2}$ delermines the physical size of the systems. or equiralent!! the fineness of the noment uni space grid.

\subsection{LONG RANGE FIELDS AND CONSERVED CHARGES}

It is common in field theories in one spatial dimension for nields to persist at large distances because of the lack of dimensions in which to spread. Among oilicr consequences. it leads almost trivially to confinement for both $Q E D$ and $Q C D$. and to severe infrared divergences associated with massless scalars which prohib:i Goldstone bosons." Even in four dimensions, because they single out oule directivi: axia! gauges such as $A^{3}=0$ or $A^{+}=0$ also lead to persistent fields. ${ }^{[8]}$ is a resill. 
boundary terms may not be discarded arbitrarily, and must be taken into account during quantization, particularly in two dimensions. ${ }^{[0.10]}$

Light-cone quantization introduces this problem even for free fermions; when massless, physical degrees of freedom live on the boundaries in $z^{-}$. This simple case in two dimensions will be discussed first in some detail, because it is simple and the necessary outcome is known, and iecause it leads to a framework for banding related problems in less trivial systems. The approach here will follow that of McCartor," who hes applied it to quantize the massless Schwinger Model on the light-cone.

To cope with fields which may not diminish sufficiently at large distances to discard, the system may be studied in a box of lesgth $2 L$ in hoth $x^{-}$and $x^{+}$ directions. In this way, boundary terms may be explinitly incorporated.

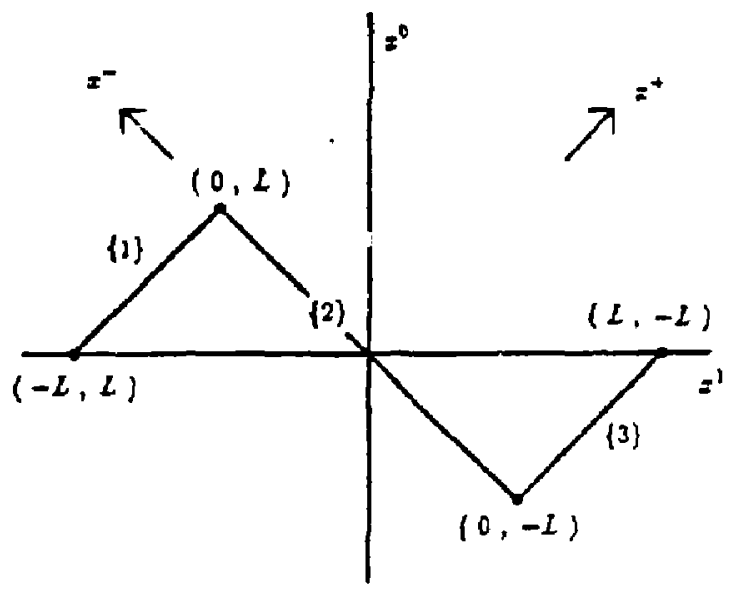

Figure 1. Surfaces Used to Defipe Charges.

The objective in quantizing a theory on the light cone is to cast it in terms of 
convenient degrees of freedom while leaving intact its physical content. With this in mind, a time-independent charge $Q$ derived from a conserved current $j^{\mu}$ which is conventionally defined on an initial surface $x^{0}=0$,

$$
Q=\int_{-\sqrt{2} L}^{\sqrt{2} L} d x^{1} j^{0}\left(0, x^{1}\right)
$$

may be rewritten in terms of $j^{\mu}$ at $x^{+}=0$ provided that the flow through the boundaries $\{1\}$ and $\{3\}$ in Fig. 1 is accounted for:

$$
Q=\frac{1}{2} \int_{-L}^{L} d y^{-} j^{+}\left(0, y^{-}\right)+\frac{1}{2} \int_{-L}^{0} d y^{+} j^{-}\left(y^{+}, L\right)+\frac{1}{2} \int_{0}^{L} d y^{+} j^{-}\left(y^{+},-L\right)
$$

If the currents drop off sufficiently in $x^{-}$, this reduces to the more usual light-cone definition; this will not always be the case. The charge $Q\left(I^{+}\right)$at an arbitrary $I^{+}$. provided that it is defined by integrating on a surfacs extended to the $x^{0}=0$ axis. will be independent of $x^{\mu}$ and equivalen: to $Q$ defined as in Eq. (1.2). Specifically.

$$
Q\left(x^{+}\right)=\frac{1}{2} \int_{-L}^{L} d y^{-} j^{+}\left(x^{+}, y^{-}\right)+\frac{1}{2} \int_{-L}^{\tau^{+}} d y^{+} j^{-}\left(y^{+}, L\right)+\frac{1}{2} \int_{x^{+}}^{L} d y^{+} j^{-}\left(y^{+},-L\right) .(1.4
$$

satisfies $\partial_{+} Q\left(x^{+}\right)=0$ identically by $\partial_{\mu} j^{\mu}=0 ;$ the $z^{+}$derivatives of the last two terms explicitly cancel the surface terms from the first.

With this prescription. the conserved momenta $P^{\mu}$ in terms of the energ:momentum tensor $\Theta^{\mu \nu}$ defined at $x^{+}=0$ are then

$$
P^{\mu}=\frac{1}{2} \int_{-L}^{L} d y^{-} \Theta^{+\mu}\left(0, y^{-}\right)+\frac{1}{2} \int_{-L}^{0} d y^{+} \Theta^{-\mu}\left(y^{+}, L\right)+\frac{1}{2} \int_{0}^{L} d y^{+} \Theta^{-\mu}\left(y^{+},-L\right) .(1.51
$$




\subsection{QUANTIZATION OF FREE FERMIONS}

The Lagrangian density for free fermions,

$$
\mathcal{L}=\bar{\psi}\left(\frac{1}{2} i \stackrel{\vec{p}}{-}-m\right) \dot{\psi}
$$

in two dimensions and light-cone coordinates and explicitly in terms of left and right spinor components is

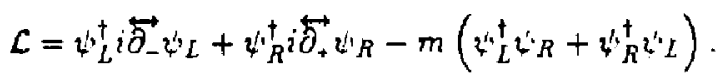

The corresponding equations of motion are

$$
\begin{aligned}
& i \partial_{+} \psi^{\prime} R=\frac{m}{2} L_{L} \\
& i \partial_{-} \dot{L}_{L}=\frac{m}{2} L^{\prime} R
\end{aligned}
$$

arid both components satisfy

$$
\left(\partial^{2}+m^{2}\right) L^{2} L \cdot R=0 .
$$

In standard light-cone guantization. independent fields are specified at $x^{+}=0$. However, $x^{+}=0$ (as well as $x^{-}=0$ ) is a characteristic surface of the wave equation: as such, it is not in general possible to specify adequate initia! conditions lor commutation relaions to quantize them/ on $x^{+}=0$ alone. Sce. For example. Refis. [12] to [14]. For the specific case when $m=0, \psi_{L}$ becontes a function of $x^{+}$alone. and initial conditions must be given on a surface (that is, a line in two dimensions; at no pout: tangent to lines of constant $x^{+}$, such as the surface $x^{-}=0$. 
To quantize this system in a way which incorporates the $m=0$ case naturally and also takes advantage of the surfaces in Fig. 1 on which charges are defined. the system is confined to a box of length $2 L$ in $x^{+}$and $x^{-}$. By using Eqs. (1.8) and (1.9), it is simple to jdentify which fields are in lependent; that is, which initial confitions are appropriate. Suppose that $\psi_{R}$ is specified along surface $\{2\}$ at $x^{+}=0$. On this surface, Eq. (1.S) is a constraint. By fixing $\psi_{L}$ at one point on this surface, at $(0,-L)$ for example, $\psi_{L}$ may be determined along it by integration. Now that both $\psi_{R}$ and $\psi_{L}$ are known on $\{2\}$, Eq. (1.8) may be used to evolve $\psi^{\prime} R$ to the surface $x^{+}=0+\Delta x^{+}$. Again, fixing $\psi_{L}$ at an intersecting point on surface \{3\} allows $\psi^{4} L$ to be integrated out along $x^{+}=\Delta x^{+}$by Eq. (1.y), and so on. It is sufficient by construction then to specify $\psi_{R}$ on $x^{+}=0$ and $\psi_{L}$ on surfaces $\{1\}$ and (3) (or alternatively on $x^{-}=0$ ) to fully determine the solutions throughout the box. To quantize, the conjugate momenta which generate evolution off of these surfaces,

$$
\pi_{R}=\frac{\delta \mathcal{L}}{\delta\left(\partial_{+} \psi_{R}\right)}=i \zeta_{R}^{\dagger}
$$

on surface $\{2\}$, and

$$
\pi_{L}=\frac{\delta \mathcal{L}}{\delta\left(\partial_{-} \psi_{L}\right)}=i \psi_{L}^{\dagger}
$$

on $\{1\}$ and $\{:\}$, are assigned commutation relations

$$
\left\{\pi_{R}\left(x^{-}\right), \psi_{R}^{*}\left(y^{-}\right)\right\}=-i \delta\left(x^{-}-y^{-}\right)
$$

and

$$
\left\{\pi_{L}\left(x^{+}\right), v_{L}\left(y^{+}\right)\right\}=-i \varepsilon\left(x^{+}-y^{+}\right) .
$$

To realize Eq. (1.13) on $x^{+}=0, \zeta^{\prime}$ may be expanded in normal modes after 
imposing appropriate boundary conditions. Periodic boundary conditions require a state of momentum $k^{+}=0$, where $k^{+}$is conjugate to $x^{-}$, for completeness, but such a state cannot be a solution of the free equation of motion $k^{+} k^{-}=m^{2}$ unless $m^{2}=0$. However, antisymmetric boundary conditions allow $\psi_{R}$ to be expanded in a set of such solutions which is also complete..$^{[13]}$ Then

$$
\psi_{R}\left(0, x^{-}\right)=\frac{1}{\sqrt{2 L}} \sum_{n=\frac{1}{2}, \frac{3}{2} \ldots}^{\infty}\left(b_{n} e^{-i \frac{n \pi}{L} x^{-}}+d_{n 1}^{\dagger} \epsilon^{\prime \frac{n \pi}{L} x^{-}}\right)
$$

with anticommutation relations

$$
\left\{b_{n}, b_{m}^{\dagger}\right\}=\left\{d_{n}, d_{m}^{\dagger}\right\}=\delta_{n, m} ;
$$

the remaining anticommutators are zero.

To treat $\psi_{L}$ and $\psi_{R}$ symmetrically, $\psi_{L}$ is expanded on $\{1\}$ and $\{3\}$ such thai

$$
\psi_{L}\left(x^{+}, \pm L\right)=\frac{1}{\sqrt{2 L}} \sum_{n=\frac{1}{2}, \frac{3}{2}, \ldots}^{\infty}\left(\tilde{b}_{n} e^{-i \frac{n \tau}{L} x^{+}}+\tilde{d}_{n}^{\dagger} e^{i \frac{n-}{L} x^{+}}\right) .
$$

for which the argument $x^{-}$is $+L$ for $x^{+}<0,-L$ for $x^{+}>0$. The operators $\dot{b}$ and $\dot{d}$ and their adjoints satisfy similar anticommutation relations, but commute with $b$ and $d$ because they represent independent degrees of freedon.

The energy-moment um tensor for free fermions,

$$
\Theta^{\mu \nu}=\frac{1}{4} \dot{4}\left(i \overleftrightarrow{\partial^{\mu}} \gamma^{\nu}+i \overleftrightarrow{\partial^{\prime \prime}} \gamma^{\mu}\right) \zeta-g^{\mu \nu} \mathcal{L}
$$


has components

$$
\begin{gathered}
\Theta^{++}=2 \psi_{R^{i}}^{\dagger}{\overleftrightarrow{\partial_{-}}}_{\psi^{\prime} R} \\
\Theta^{--}=2 \psi_{L}^{\dagger} i \vec{\partial}_{+} \psi_{L} \\
\Theta^{-+}=\Theta^{+-}=m\left(\psi_{L}^{\dagger} \psi_{R}+\psi_{R^{L^{\prime}} L}^{\dagger}\right) .
\end{gathered}
$$

The evolution operators are then, by Eq. (1.5)

$$
\begin{aligned}
& P^{+}=\int_{\{2\}} d x^{-} \psi_{R}^{\dagger} i \vec{\partial}_{-} \psi_{R}+\frac{1}{2} m \int_{\{1\}+(3)} d x^{+}\left(\psi_{L}^{\dagger} \psi_{R}+\psi_{R}^{\dagger} \psi^{i} L\right) \\
& P^{-}=\frac{1}{2} m \int d x^{-}\left(\dot{\psi}_{L}^{\dagger} \psi_{R}+\psi_{R}^{\dagger} \psi^{\prime} L\right)+\int d x^{+} \psi_{L}^{\dagger} i \vec{\partial}_{+} \psi_{L}
\end{aligned}
$$

For the special case where $m=0$, substitution of Eq. (1.15) into

$$
P^{+}=\int_{-L}^{I} d x^{-} \psi_{R^{\dagger}}^{\dagger} \vec{\partial}_{-\psi^{+}} R\left(x^{+}=0\right)
$$

gives

$$
P^{+}=\sum_{n=\frac{1}{2}, \frac{3}{2}, \ldots}^{\infty}\left(\frac{2 \pi n}{l}\right)\left(b_{n}^{\dagger} b_{n}+d_{n}^{\dagger} d_{n}-1\right)
$$

the divergent vacuum momentum may be discarded. Because $4 \cdot 2$ is expanded in Eq. (1.17) in deliberate analogy to 2 is.

$$
P^{-}=\int_{-L}^{0} d x^{+} \psi_{L}^{\dagger} i \vec{\partial}_{+} L_{L} L\left(x^{+}, L\right)+\int_{0}^{L} d x^{+} \psi_{L^{+}}^{\dagger} i \overleftrightarrow{\partial}_{\tau} L L\left(x^{+},-L\right)
$$


produces

$$
P^{-}=\sum_{n=\frac{1}{2}, \frac{3}{2}, \ldots}^{\infty}\left(\frac{2 \pi n}{L}\right)\left(\bar{b}_{n}^{\dagger} \tilde{b}_{n}+\tilde{d}_{n}^{\dagger} \tilde{d}_{n}-1\right)
$$

Because the untilded and tilded operators anticommute, states composed of the former have $P^{-}=0$, while the latter satisfy $P^{+}=0$. Clearly then the $b_{n}^{\dagger}$ and $d_{n 1}^{\dagger}$ create massless, right-moving particles of equal-time momentum

$$
P^{1}=\frac{1}{2}\left(P^{+}-P^{-}\right)=\frac{1}{2} P^{+}=\frac{\pi n}{L}
$$

and energ:

$$
P^{0}=\frac{1}{2}\left(P^{+}+P^{-}\right)=\frac{1}{2} P^{+}=\frac{\pi n}{L},
$$

while the $\dot{b}_{n}^{\dagger}$ and $\tilde{d}_{n}^{\dagger}$ have

$$
P^{1}=-\frac{1}{2} P^{-}=-\frac{\pi n}{L}
$$

and

$$
P^{0}=\frac{1}{2} P^{-}=\frac{\pi n}{L}
$$

and are left-moving. Also, the vacuum values subtracted correspond to

$$
\begin{aligned}
& \left\langle P^{0}\right\rangle=\frac{1}{2}\left\langle P^{+}\right\rangle+\frac{1}{2}\left\langle P^{-}\right\rangle=-\sum\left(\frac{2 \pi n}{L}\right) \\
& \left\langle P^{1}\right\rangle=\frac{1}{2}\left\langle P^{+}\right\rangle-\frac{1}{2}\left\langle P^{-}\right\rangle=0
\end{aligned}
$$

as usual, only the energy $P^{0}$ requires a subtraction. The degrees of freedon atid physica! interpretation of the usual equal-time quantization have been preserved: 
specifically, both left-and right-moving massless fermions have been retained. This is particularly important when defining the vector and axial vector currents

$$
j^{\mu}=: \bar{\psi} \gamma^{\mu} \psi^{\prime}: \quad \text { and } \quad j_{5}^{\mu}=: \bar{\psi} \gamma^{5} \gamma^{\mu} \psi^{\mu}:
$$

with components

$$
j_{(5)}^{+}=2: \psi_{R}^{\dagger} \psi^{\prime} R_{R}: \quad \text { and } \quad j_{(5)}^{-}=(-) 2: \psi_{R^{2}}^{\dagger} \cdot R:
$$

Their corresponding charges are

$$
Q_{(5)}=\frac{1}{2} \int_{-L}^{L} d x^{-} j_{(5)}^{+}\left(0, x^{-}\right)+\frac{1}{2} \int_{-L}^{0} d x^{+} j_{(5)}\left(x^{+}, L\right)+\frac{1}{2} \int_{0}^{L} d x^{+} j-(5)\left(x^{+},-L\right) .
$$

Note that if the $\psi_{2} 2$ degrees of freedom were not retained, $j^{\mu}$ and $j_{5}^{\mu}$ would be indistinguishable. In momentum space,

$$
Q_{(5)}=\sum_{n=\frac{1}{2}, \frac{3}{2}, \ldots}^{\infty}\left(b_{n}^{\dagger} b_{n}-d_{n}^{\dagger} d_{n}\right) \underset{(-)}{+}\left(b_{-n}^{\dagger} b_{-n}-d_{-n}^{\dagger} d_{-11}\right)
$$

where the left-moving $\dot{b}_{n}$ and $\dot{d}_{n}$ have been re-written as $b_{-n}$ and $d_{-n}$.

When the fermion mass is not identically zero. then in $P^{-}$, for example, from Eq. (1.20), there is an adcitional term proportional to $m$ which requires both $6 \cdot R$ and $\psi^{\prime} L$ along the contour. On the surface $x^{+}=0$, where $\psi_{R}$ is specified. $\psi_{L}$ may be determined by Eq. (1.9). The most generai solution is

$$
\psi_{L}\left(0, x^{-}\right)=-\frac{1 m}{4} \int_{-L}^{L} d y^{-} \epsilon\left(x^{-}-y^{-}\right) \psi^{\prime} R\left(0, y^{-}\right)+\frac{1}{2}\left[\psi_{L}(0, L)+\psi_{L}(0,-L)\right] \cdot(1.34)
$$

The last boundary term depends on conditions chosen on the surfaces perpendicular to $x^{+}=0$ and is independent of $4 k$. Eq. $(1.34)$ is true independent of discretization. 
and the boundary term is essential so that $\psi_{L}\left(x^{-}\right)$transforms correctly under symmetry transformations such as Lorentz boosts.

Substitution into the mass term in Eq. (1.20) for $P^{-}$gives, from the term with $\psi_{R}$ in Eq. (1.24), the usual light-cone energy

$$
\sum_{n=\frac{1}{2}, \frac{3}{2}, \ldots}^{\infty}\left(\frac{m^{2}}{p_{n}^{+}}\right)\left(b_{n}^{\dagger} b_{n}+d_{n}^{\dagger} d_{n}\right)+\text { const }
$$

with $p_{n}^{+} \equiv 2 \pi n / L$. The boundary term contributes

$$
\frac{m}{3}\left[\int_{-L}^{L} d x^{-} \psi^{\dagger} \cdot\left(0, x^{-}\right)\right] \frac{1}{2}\left[\psi^{\prime} L(0, L)+\psi \cdot L(0,-L)\right]+\text { conj. }
$$

or in mornentum space,

$$
\frac{m}{2 \pi} \sum_{n} \frac{(-1)^{n}}{n}\left(b_{n}^{\dagger}+d_{n}\right) \sum_{\ell}\left(\tilde{b}_{\ell}^{\dagger}+\dot{d}_{\ell}\right)+\operatorname{con} j
$$

For finite $L$, this represents a well-defined interaction between the formerly massles: left-and right-moving quanta. However, in the continuum limit the integral over $\psi_{R}$ (or the sum over $n$ ) reduces to a term proportional to $b_{0}^{t}+d_{0}$, which contributes infinity from the factor $\mathrm{m}^{2} / \mathrm{p}^{+}$in Eq. (1.35). Therefore, from this term, all of the $\hat{b}$ and $\dot{d}$ couple to states which contain $b_{0}$ or $d_{0}$, witb $P^{+}=0$ and $P^{-}=\propto$.

An analogous result is obtained for the operator $P^{+}$, but with plus and minus. and tilded and untilded operators reversed. In the continuum linit for massive fermions, then, the Poincare algebra must be built either on the tilded or untilded operators. The two are related by parity, but separated by an infinite esserg: barrier, and the choice determines whether $x^{+}=$constant or $x^{-}=$constant is 
treated as the usual light-cone quantization surface. It is at this point, then. that the well-known phenomenon occurs in which light-cone quantization removes half of the fermionic degrees of freedom. Apparently, this is only appropriate for massive fermions.

The final result is physically sensible. In the continuum limit, the surface $x^{+}=0$ is inadequate for specifying initial conditions for massless particles because left-moving particles can rug parallel to it; conditions on a surface such as $x^{-}=0$ must also be specified. When massive, however, any particle with finite momenturn will intercept this surface at some point. For extremely energetic particles. however. this point may be very far away in $x^{-}$, so this statement is only true if the length $2 L$ of $x^{-}$included is infinite. In fact, it may be shown explicitly that the surface $x^{+}=0$ is suffcient to specify solutions to the massive wave equation by mapping these solutions onto those completely specified on a space-like surface, such as $z^{0}=0$.

Because the goal of this numerical work is to obtain continum linit solutions, the fermion masses will be kept finite, terms such as that in Eq. (1.3i) wi:i be explieitly excluded, and massless fermions will be considered as a limit.

\subsection{QUANTIZATION OF SE $(N)_{1+1}$}

An $S E\left(N^{\prime}\right)$ gauge theory is defined by the Lagrangian

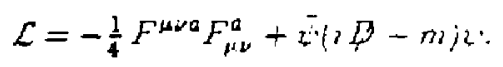

$F_{\mu \nu}^{a}$ is the field strength tensor

$$
F_{\mu \nu}^{a}=\partial_{\mu} \cdot A_{\nu}^{a}-\partial_{\nu} \cdot A_{j}^{u}-g f^{r a b c} \cdot A_{\mu}^{b} \cdot A_{\nu}^{c}
$$


and the covariant derivative is defined as

$$
i D_{\mu}=i \partial_{\mu}-g A_{\mu}^{a} T^{a}
$$

Operator ordering is potentially important; for example, the fields $A_{\mu}^{a}$ will not conmute after quantization, and in expressions such as Eq. (1.39) should be considereu as appropriately symmetrized.

The gauge group generators satisly

$$
\left[T^{a}, T^{b}\right]=i f^{a b c} T^{c}
$$

and are normaiized such that

$$
\operatorname{Tr} T^{a} T^{b}=\frac{1}{2} \delta^{a b}
$$

tbe structure constants $f^{a b c}$ are fully antisymmetric. The completeness relation:

$$
\left[T^{a}\right]^{\prime}\left[T^{a}\right]_{\ell}^{k}=\frac{1}{2 . T}\left(N \varepsilon_{j}^{k} \varepsilon_{l}^{i}-\varepsilon_{j} \varepsilon_{l}^{k}\right)
$$

will be useful when constructing the Hamiltonian. The second term on the righ: is a result of the tracelessness of the generators, and is not present for the gauge group $C(: i)$.

In two dimensions, the fermion field (in a representation in which $a^{5}$ is diagolial,

$$
u=\left(\begin{array}{l}
\tau(I)_{c} \\
\zeta R(I)_{c}
\end{array}\right)
$$

is a two-component spinor in the fundamestal representation, $L$ and $R$ ir cical chirality, which, for massless fermions specifies only disection. In that casie $\because \%$ 
is a function of only $x^{+}, \psi_{R}$ of $x^{-}$. The chiral $\gamma$ matrix representation is used throughout. Explicitly,

$$
\begin{array}{ll}
\gamma^{6}=\left(\begin{array}{ll}
0 & 1 \\
1 & 0
\end{array}\right) & \gamma^{1}=\left(\begin{array}{cc}
0 & 1 \\
-1 & 0
\end{array}\right) \\
\gamma^{+}=\left(\begin{array}{ll}
0 & 2 \\
0 & 0
\end{array}\right) & \gamma^{-}=\left(\begin{array}{ll}
0 & 0 \\
2 & 0
\end{array}\right)
\end{array}
$$

with the chiral projection operators

$$
\begin{aligned}
& \frac{1}{2}\left(1-\gamma^{5}\right)=\frac{1}{4} \gamma^{+} \gamma^{-}=\left(\begin{array}{ll}
1 & 0 \\
0 & 0
\end{array}\right) \\
& \frac{1}{2}\left(1+\gamma^{5}\right)=\frac{1}{4} \gamma^{-} \gamma^{+}=\left(\begin{array}{ll}
0 & 0 \\
0 & 1
\end{array}\right) .
\end{aligned}
$$

As usua].

$$
\left\{\gamma^{\mu}, \gamma^{\nu}\right\}=2 g^{\mu \nu} .
$$

A useful gauge choice is $A^{+a}=0$. As is generally the case for axial gauges, there are neither ghosts nor negatively normed gauge bosons, so the Fock space quanta. and therefore the wavefunction constituents are physical and positively normed. In particular, these gauges allow constraint equations to be solved for explicitiy ai the Lagrangian level, so that only physical degrees of freedom are quantized and appear in the Hamilionian and space of states. Also. in $1+1$ dimensions. in gauge choice is Lorentz (but not parity) invariant. 
The equations of motion are then

$$
\begin{gathered}
i \partial_{-} \psi_{L}=\frac{1}{2} m \iota \psi^{\prime} R \\
-\partial_{-}^{2} A^{-a}=g \psi_{R}^{\dagger} T^{a} \psi^{\prime} R \\
i \partial_{+} \zeta^{\prime} R=\frac{1}{2} g \cdot A^{-a} T^{a} \psi_{R}^{\prime}+\frac{1}{2} m \psi_{L} \\
\partial_{+} \partial_{-} A^{-a}=g r_{L}^{\dagger} T^{a} \psi_{L}-\frac{1}{2} g f^{a b c} \partial_{-} \cdot A^{-b} \cdot A^{-c} .
\end{gathered}
$$

Here and throughout, when not shown, color indices on the fermion fields are injplied. As was the case with free fermions, it is important that all real degrees of freedom are identified, and in particular, that those associated with boundary terms are not discarded. This is necessary in axial gauges even for equal-time quantization and in four dimeusions.

To quantize $S\left[\left(N^{*}\right)_{1+1}\right.$, charges vill again be defined to account for bound. ary terms and will be explicitly conserved. As discussed in Refs. [10] and [E]. boundary terms for the gauge feld appear which cannot be made to vanisto at infinity, and which are related to the residual gauge symmetry under $x^{-}$-indepcuden: transformation.

In this gauge, the Lagrangian reduces to

$$
\begin{aligned}
& \mathcal{L}=\frac{1}{2}\left(\partial_{-} A^{-a}\right)^{2}-g L_{R}^{\dagger} A^{-a} T^{a}{ }^{2} \cdot R
\end{aligned}
$$

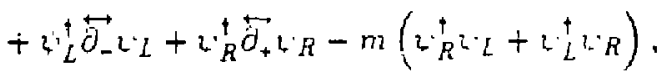


while the elements of the energy-momentum tensor become

$$
\begin{aligned}
& \Theta^{++}=2 \psi^{\prime} R^{i} \vec{\partial}_{-} \iota_{R}
\end{aligned}
$$

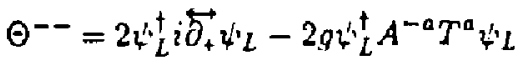

$$
\begin{aligned}
& \Theta^{-+}=\Theta^{+-}=\left(\partial_{-} A^{-a}\right)^{2}-m\left(\psi_{R}^{\dagger} \psi_{L}+\psi_{L}^{\dagger} \psi R\right) .
\end{aligned}
$$

Also,

$$
\begin{aligned}
& j^{+a}=2 g l_{R}^{\dagger} T^{a} \psi_{R} \\
& j^{-a}=2 g b_{L}^{\dagger} T^{a}{ }_{L} \cdot L-g f^{a b c} \partial_{-} A^{-b} A^{-c}
\end{aligned}
$$

are the conserved currents associated with global gauge transformations.

The generators $P^{+}$and $P^{-}$will be constructed in the same way as for frec fermions, using Eq. (1.5). Because they are explicitly independent of $x^{+}$. they may be defined at $x^{+}=0$, along the contours in Fig. 1. Including gauge fields.

$$
\begin{aligned}
& P^{+}=\int_{-L}^{L} d y-\psi_{R}^{t} i \vec{\partial}_{-L_{R}} \\
& +\int_{-L}^{L} d y+\left[\frac{1}{2}\left(\partial_{-} A^{-\alpha}\right)^{2}+\frac{m}{2}\left(L \cdot R^{2} \cdot L+2 \cdot L^{\dagger} L \cdot R\right)\right]
\end{aligned}
$$

and

$$
\begin{aligned}
& P^{-}=\int_{-L}^{L} d y^{-}\left[\frac{1}{2}\left(\partial_{-} A^{-a}\right)^{2}+\frac{m}{2}\left(r^{\dagger} R^{\dagger} \cdot L+\tau^{\dagger} L^{\dagger} \cdot R\right)\right] \\
& +\int_{-L}^{L} d y+\left[V_{L}^{\dagger} i \ddot{\theta}_{+} i_{L}-g L_{L}^{\dagger} \cdot A^{-a} T^{a} V_{L}\right] .
\end{aligned}
$$

The integrals over $y^{+}$are to be understood as over coniours $\{1\}$ and $\{3\}$ in: $F:$ I 
throughout this discussion. In order to quantize, the degrees of freedom may be determined from the equations of motion, Eqs. (1.48) to (1.51).

Again, consider the system in a box of length $2 L$ in $x^{+}$and $x^{-}$. Specifying $\psi_{R}$ along $x^{+}=0$ allows $\psi_{L}, A^{-a}$ and $\partial_{-} A^{-a}$ to be determined from Eqs. (1.4S) and (1.4y), which are constraint equations on this surface, provided that they are specified at one point; for example, at $\left(x^{+}=0, x^{-}=-L\right)$. Knowing these along $I^{+}=0$, Eq. (1.50) gives the evolution of $1 / R$ to the next $x^{+}+\Delta x^{+}$surface.

Within this box, then, it is sufficient to specify $\psi^{\prime} R$ at $x^{+}=0$ (that is, surface $\{2\}$ of Fig. 1$)$, and $A^{-a}, \partial_{-} A^{-a}$ and $\psi_{L}$ on surfaces $\{1\}$ and $\{3\}$ : these are then the independent degrees of freedom.

Two comments are warranted. First, differentiating Eq. (1.51) with respect to $x^{-}$shows it to be consistent with Egs. (1.48) to (1.50). but only up to a boundary term independent of $x^{-}$. Second, setting $A^{+a}=0$ does not complesely fix the gauge, as any $x^{-}$-independent gauge transformations preserve $A^{+a}=0$. This residual freedom may be used to $\mathrm{fx} A^{-a}$ on one boundary. boundary, $A^{-a}$ would then be given in terms of other physical quantities. Ii will prove adrantageous, bowever, to expand the phase space to include $A^{-a}$ as a rhysical degree of freedom. and to eliminate it at later stage.

On surfaces of constant $x^{+}$, Eqs. (1.48) and (1.49) are constraint equations and may be solved explicitly:

$$
\begin{aligned}
\psi^{\prime} L(x)=-\frac{m}{4} \int_{-L}^{L} d y-\epsilon\left(x^{-}-y^{-}\right) L_{R} \cdot\left(x^{+}, y^{-}\right) \\
\\
+\frac{1}{2}\left[L \cdot L\left(x^{+} . L\right)+L \cdot L\left(x^{+},-L\right)\right]
\end{aligned}
$$




$$
\begin{aligned}
\partial_{-} A^{-a}(x)=-\frac{g}{2} \int_{-L}^{L} d y^{-} \epsilon\left(x^{-}-y^{-}\right) \psi^{\prime} R_{R}^{\dagger} T^{a} \cdot R\left(x^{+}, y^{-}\right) \\
\\
\quad+\frac{1}{2}\left[\partial_{-} A^{-\alpha}\left(x^{+}, L\right)+\partial_{-} A^{-a}\left(x^{+},-L\right)\right]
\end{aligned}
$$

and

$$
\begin{aligned}
A^{-a}(x)= & -\frac{g}{2} \int_{-L}^{L} d y^{-}\left|x^{-}-y^{-}\right| \psi_{R}^{\dagger} T^{a} \psi_{\psi_{R}}\left(x^{+}, y^{-}\right) \\
& +\frac{1}{2} x^{-}\left[\partial_{-} A^{-a}\left(x^{+}, L\right]+\partial_{-} A^{-a}\left(x^{+},-L\right)\right\} \\
& +\frac{1}{2} L Q_{R}^{a(0)}\left(x^{+}\right)+\frac{1}{2}\left[A^{-a}\left(x^{+}, L\right)+A^{-a}\left(x^{+},-L\right)\right\}
\end{aligned}
$$

where the moments $Q_{R}^{a(n)}$ are defined as

$$
Q_{R}^{a(n)} \equiv \int_{-L}^{L} d y^{-}\left(y^{-}\right)^{n} L_{R}^{\dagger} T^{a} L_{R}^{\prime}\left(x^{+}, y^{-}\right)
$$

In spite of the appearance of $L$, these solutions are completely geneia!. as no boundary conditions have been imposed. Provided that the fields at $\pm L$, as well as $L$ jtself, transform as usual, these are fully izvariant under Lorentz and residua! gauge transformations. Also. from Eqs. (1.5i) $20(1.59)$, these fields at $r^{-}==L$ are related by

$$
\begin{gathered}
\left.\psi_{L}\left(x^{+}, L\right)-\ell_{L}\left(x^{+},-L\right)=-i \frac{m}{2} \int_{-L}^{L} d y^{-} \zeta R^{i} x^{+}, y^{-}\right) \\
\partial_{-} A^{-a}\left(x^{+}, L\right)-\partial_{-} A^{-a}\left(x^{+},-L\right)=g Q_{\dot{R}}^{a L_{1}}
\end{gathered}
$$


and

$$
\begin{aligned}
A^{-a}\left(x^{+}, L\right)-A^{-a}\left(x^{+},-L\right)= & g Q_{R}^{a(1)} \\
& +L\left[A^{-a}\left(x^{+}, L\right)+A^{-a}\left(x^{+},-L\right)\right] .
\end{aligned}
$$

Eq. (1.6?) in particular gives the integral form of Gauss' Law. 'Therefore. 2. $A^{-a}$, and $\partial_{-} A^{-a}$ are independent variables at only one boundary. To completely solve the equations of norion they must be specified at either $L$ or $-L$, but not both independently, and in particular, none of these be set to zero at both ends. As a convention. when $x^{+}=0$, the average of the two boundarg terms, such as $(1 / 2)\left[A^{-a}(0, L)+A^{-a}(0,-L)\right\}$, will be treated as the independent variable: see Fig. 1.

On surfaces $\{1\}$ and $\{3\}, \psi_{L}, A^{-a}$, and $\partial_{-} A^{-a}$ are the independent degrees of freedom, and Eq. (1.50) becomes a constraint equation. In principle, $\iota^{\prime} R$ may be solved for by inverting $i \partial_{+}-(g / 2) A^{-a} T^{a}$. This may be expressed iteratively. '.ut the explicit solution will not be needed.

The independent degrees of freedom may now be quantized by postuiatirg canonical commutation relations for the momenta which generate translations per. pendicular to the surfaces on which they are defined. Then, at $x^{+}=0$ (surface $\{2\}\}$,

$$
\pi_{R}=\frac{\delta L}{\delta\left(\partial_{+} \psi^{\prime} R\right)}=i \zeta_{R}^{\dagger}
$$

while on $\{1\}$ and $\{3\}$.

$$
\begin{aligned}
& \pi_{L}=\frac{\delta \mathcal{L}}{\delta\left(\partial_{-} \psi^{\prime}\right)}=i \zeta_{L}^{\dagger} \\
& \pi_{A}^{a}=\frac{\delta \mathcal{L}}{\delta\left(\partial_{-} A^{-a}\right)}=\partial_{-} A^{-a} .
\end{aligned}
$$


The anticommutators for $\psi_{R}$ and $\psi_{c}$ are given in Eq. (1.13) and (1.14). In addition.

$$
\left.\left[\pi_{A}^{a}\left(x^{+}\right), A^{-b}\left(y^{+}\right)\right]\{]\right\}+\{3\}=-i \delta^{a b} \delta\left\{x^{+}-y^{+}\right] .
$$

As usual, felds which are independent commute. The independent fields may be expanded in creation and annihilation operators such that the (anti)commutation relations are realized, and from these the Fock space may be constructed.

The final result of solving for dependent degrees of freedom where appropriate and substituting in Eqs. (1.55) and (1.56) at $x^{+}=0$ is that

$$
\begin{aligned}
& P^{-}=-\frac{e m^{3}}{4} \int d x^{-} d y^{-} \epsilon\left(x^{-}-y^{-}\right) \psi_{R}^{\dagger}\left(x^{-}\right) \psi_{R}\left(y^{-}\right) \\
& -\frac{q^{2}}{4} \int d x^{-} d y^{-} \psi_{R}^{\dagger} T^{a} \psi_{R}\left(x^{-}\right)\left|x^{-}-y^{-}\right| \psi_{R}^{\dagger} T^{a} \psi^{\prime}{ }_{R}\left(y^{-}\right) \\
& +\int d y^{+}\left(\psi_{L}^{\dagger} i \vec{a}_{+} \psi_{L}\left(y^{+}\right)-g \psi_{L}^{\dagger} A^{-a} T^{\alpha} \psi_{L}\left(y^{+}\right)\right) \\
& +\frac{m}{2}\left[\int d x^{-} \psi_{R}^{\dagger}\left(x^{-}\right)\right] \psi_{L}(0)+\frac{m}{2} \psi_{L}^{\dagger}(0)\left[\int d x^{-} \imath_{R}\left(x^{-}\right)\right] \\
& +\frac{q^{2}}{4} L Q_{R}^{a(0)} Q_{R}^{a(0)}+L \pi_{A}^{a} \pi_{A}^{a}+g \pi_{A}^{a} Q_{R}^{a(1)}
\end{aligned}
$$

and

$$
\begin{aligned}
& P^{+}=\int d I^{-} v_{R}^{+} i \vec{\partial}_{-} \psi_{R}+\frac{1}{2} \int d I^{+}\left(\pi_{A}^{a}\right)^{2}
\end{aligned}
$$

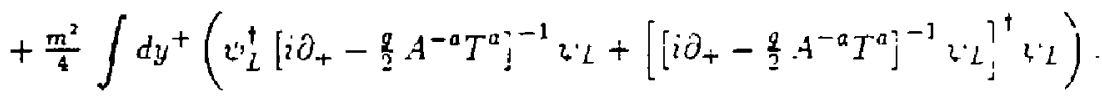

Also, the total gauge charge defined at $x^{+}=0$ is

$$
Q^{a}=Q_{R}^{a(0)}+\int d y+\left(b_{L}^{\dagger} T^{a} L_{L}-\frac{1}{2} f^{a b c} \pi_{A}^{b} \cdot A^{-r}\right) .
$$


and the Lorentz boost generator is

$$
\begin{aligned}
& \mathbb{\Omega}_{B}=-\frac{1}{2} A^{+-} \\
& =\frac{1}{4} \int d y^{-}\left(y^{-} \Theta^{++} \cdot x^{+} \Theta^{+-}\right)+\frac{1}{4} \int_{\{1\}+\{3 \mid} d y^{+}\left(x^{-} \Theta^{-+}-y^{+} \Theta^{--}\right) \\
& \text {(2) } \\
& \{1\}+\{3\} \\
& =\frac{1}{2} \int_{-L}^{L} d y^{-} y^{-} \psi_{R^{\dagger}}^{\dagger} \vec{\partial}_{-} \psi_{R}-\frac{1}{2} \int_{-L}^{L} d y^{+} y^{+}\left(\psi_{L}^{\dagger} i \vec{\partial}_{+} \psi_{L}-g L_{L}^{\dagger} A^{-a} T^{a} \psi_{L} L\right) \\
& -\frac{1}{2} L \int_{0}^{L} d y+\left(\frac{1}{2}\left(\partial_{-} A^{-a}\right)^{2}+\frac{m}{2} L^{2}\right)+\frac{1}{2} L \int_{-L}^{0} d y+\left(\frac{1}{2}\left(\partial_{-}, A^{-a}\right)^{2}+\frac{m}{2} l_{L}\right)
\end{aligned}
$$

with dependent fields replaced where appropriate.

The canonical momentum $\pi_{A}^{a}$ is given by $\partial_{-} A^{-a}=(1 / 2) F^{+-a}$ on the bourdary, and acts as an $x^{-}$-jndependent background cbromoelectric field. As such. it contributes to $P^{-}$in Eq. (1.67) an energy proportional to the length of the system. and couples to the first moment of the charge density, $Q_{R}^{a(1)}$.

The momenta $P^{\mu}$ generate the equations of motion Eqs. (1.45) to (1.50). and the fourth equation. Eq. (1.51), up to a boundary term. However, the price paid for treating $A^{-a}$ on surfaces $\{1\}$ and $\{3\}$ as a physical degree of freedom to allow: the simple commutation relation Eq. (1.66) is that Eq. (1.51) on the $x^{-}$boundary

$$
G^{a}\left(T^{+}\right) \equiv \partial_{+} \pi_{A}^{a}+\frac{a}{2} f^{a b c} \pi_{A}^{b} A^{-c}-g L_{L}^{\dagger} T^{a} L^{\prime} L \approx 0
$$

cannot be satisfied as an operator equation. $G^{a}\left(I^{+}\right)$is. in fact, the generator of the residual $I^{-}$-independent gauge transformation, which acts by changing th.. 
boundary conditions which would be necessary to fully fix the gauge. ${ }^{[0]}$ For example, on surface $\{3\}$, the commutator

$$
\begin{aligned}
i-i \int d x^{+} \Lambda^{a}\left(x^{+}\right) G^{0}\left(x^{+}\right), & \left.A^{-b}\left(y^{+},-L\right)\right] \\
& =\partial_{+} \Lambda^{b}\left(y^{+}\right)+\frac{g}{2} f^{b a c} \Lambda^{a}\left(y^{+}\right) A^{-c}\left(y^{+},-L\right),
\end{aligned}
$$

with $\Lambda^{a}\left(x^{+}\right)$parametrizing the residual transformation. Eq. (1.71) must then be imposed on the space of states. Because it generates residual gauge transformations, it commutes with the generators $P^{\mu}$, so that these will not move the states off this surface. Replacing

$$
\partial_{+} \pi_{A}^{a}=-\frac{i}{2}\left[\pi_{A}^{a}, P^{-}\right]=\frac{g}{2} \psi_{L}^{\dagger} T^{a} \psi_{L}
$$

this condition on matrix elements becomes

$$
\left\langle\Phi_{\text {physical }}^{\prime}\left|\left(f^{a b c} \pi_{A}^{b} A^{-c}\left(I^{+}\right)-\psi_{L}^{\dagger} T^{a} \psi L\left(x^{+}\right)\right)_{\{1\}+\{3\}}\right| \Phi_{\text {physical }}\right\rangle=0 .
$$

In this space.

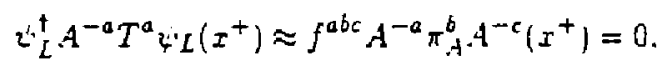

and $\psi_{L}^{\dagger} A^{-a} T^{a} q_{L}^{\prime}$ effectively vanishes from $P^{-}$and $K_{B}$. restoring some of the symmetry between $\psi_{R}$ and $\psi_{L}$. Also, Eq. (1.69) for $Q^{\alpha}$ becomes. by Eq. (1.il).

$$
\begin{aligned}
Q^{a} & \approx Q_{R}^{a(0)}+\left[\pi_{A}^{a}(-L . L)-\pi_{A}^{a}(L .-L)\right] \\
& =Q_{R}^{a(0)}+\left[\pi_{A}^{a}\left(x^{0}=0, x^{1}=-\sqrt{2} L\right)-\pi_{A}^{a}\left(x^{0}=0, x^{3}=\sqrt{2} L\right)\right] .
\end{aligned}
$$

and the gencrator of global gauge transformations may be given entirely in teum: of $\psi^{\prime \prime} R$. and $\pi_{A}^{\mathrm{u}}$ at two points on the boundary, 
This is reminiscent of equal-time quantization in the $A^{0}=0$ gauge. There the phase space is expanded to include (in four dimensions) the three spatial $A^{\prime}$ with canonical conjugates $E^{i}$. Gauss' Law, $\partial_{4} E^{1}-\rho=0$, which generates timeindependent gauge transformations, is abandoned as an operator equation but imposed on the states.

Actually, this is more than reminiscent. The equations of motion in the gauge $A^{-a}=0$ are

$$
\begin{aligned}
& i \partial_{+} \psi_{R}=\frac{m}{2} \psi_{L} \\
& i \partial_{-} \psi_{L}-\frac{\rho}{2} A^{+a} T^{0} \psi_{L} L=\frac{m}{2} \xi_{R} \\
& -\partial_{+} \pi_{A}^{a}=g \psi_{L}^{\dagger} T^{a} \psi_{L} \\
& \partial_{-} \pi_{A}^{a}+\frac{q}{2} f^{a b c} \pi_{A}^{b} A^{+c}=g \psi_{R}^{\dagger} T^{a} \xi_{R}
\end{aligned}
$$

where the canonical momentum

$$
\pi_{A}^{a}=\frac{\delta \mathcal{L}}{\delta\left(\partial_{+} \cdot A^{+a}\right)}=\partial_{+} A^{+a}
$$

The fields initialized on the boundaries $\{1\}$ and $\{3\}$ in the $A^{+a}=0$ gauge and appearing in $P^{+}$and $P^{-}$evidently correspond to those which would have been obtained by usual light-cone quantization in the gauge $A^{-a}=0$, with left and rigli: + and - interchanged; in particular, with $x^{-}$the time variable and $x^{-}=$constant an initial suriace. The condition on states, Eq. (1.74), after this interchange, corresponds to Eq. (1.80), which is Gauss' Law. 
At this stage, an apparently consistent quantization scheme has been set up in which charges and spacetime generators are conserved by construction, and boundary terms have been explicitly included. No real use has been made of the box length $2 L$, and once it is taken to infinity, the scheme is evidently also Lorent $z$ invariant. This includes the constraint, Eq. (1.74), which was obtained from a Lorentz-invariant equation of motion.

In the continuum !imit, $\psi_{L}$ on the boundaries couples to $\psi_{R}$ on!y through an infinite- $P^{-}$, zero- $P^{+}$mode, as previously discussed for free fermions. With the introduction of gauge fields, new terms have appeared in $P^{-}$, Eq. (1.67), which also become infinite in this limit. To construct a state space in $w$ hich this limit is sensible, that is, in which these terms vanish, consider first states only containing degrees of freedom associated with $\psi_{R}$. To couple these directly to $\psi_{L}$, an infinite energy $\psi_{R}^{\prime}$ particle must first he produced. In addition, $\psi_{R}$ couples to $\pi_{A}^{a}$ by the term $\pi_{A}^{a} Q_{R}^{a(1)}$ in $P^{-}$, but $Q_{R}^{a(1)}$ is a non-singlet operator. States of $\psi_{R}$ for which it does not vanish are infinite in energy due to the presence of the Casimir operator $L Q_{R}^{a(0)} Q_{R}^{a(0)}$ in $P^{-}$. This is in contrast to electrodynamics in tro dimensions. where the moment $Q^{(1)}$ can still couple to a charge singlet, and for which $\pi A$ is related to the physically relevant $\theta$ parameter. ${ }^{(19)}$ These are the only couplings between $\psi_{R}$ and the boundary degrees of freedom. Consequently, the sector of color-singlet states composed of onl $\psi^{\prime} R$ is separated from that with $\tau_{L} . A^{-a}$ and $\pi_{A}^{a}$ by an infinite-energy barrier.

The result of this very long song and dance is that a representation of the color and Poincare algebra with finite matrix elements may be constructed in terms of singlet staies of $i_{R}$ alone. In particular, the condition on the states, Eq. (1.it). is 
trivially satisfied. Another representation based on $\psi_{L}$ and corresponding to the gauge $A^{-a}=0$ could be constructed as an alternative; this will not be developed here.

Finally, in the $\psi_{R}$ sector,

$$
P^{+}=\int_{-L}^{L} d x-\psi_{R}^{\dagger} i \vec{\partial}_{-} \psi_{R}
$$

while the Hanilitonian

$$
\begin{aligned}
p^{-}= & -\frac{3 \pi x^{2}}{4} \int_{-L}^{L} d x^{-} d y^{-} \epsilon\left(x^{-}-y^{-}\right) \psi_{R^{\dagger}}^{\dagger}\left(x^{-}\right) \psi_{R}^{\prime}\left(y^{-}\right) \\
& -\frac{z^{2}}{4} \int_{-L}^{L} d x^{-} d y^{-} \psi^{\dagger}{ }_{R}^{\dagger} T^{a} \psi_{R}\left(x^{-}\right)\left|x^{-}-y^{-}\right| w_{R}^{\dagger} T^{a} \psi_{R}\left(y^{-}\right) .
\end{aligned}
$$

These will be evaluated in the sector for which the charge aperator

$$
Q^{a}=Q_{R}^{a(0)}=\int_{-L}^{L} d x^{-} \psi_{R}^{\dagger} T^{a} w_{R}
$$

vanishes. When not otherwise indicated. the products of fields $L_{R}^{\dagger} T^{o} \psi_{R}$. which come from: the normal-ordered current $j^{+a}$, are independently normal-ordered. as in $P^{-}$and $Q^{a}$ above. 


\subsection{DISCRETE HAMILTONIAN, MOMENTLM AND BOOST OPER.ATORS}

As is typically done, the anticommutation relation for $\psi_{R}(x)$ at $x^{+}=0$, Eq. (1.13), may be realized by expanding $\tau^{\prime} R$ in solutions of the free equations of motion,

$$
\psi_{R}\left(0, x^{-}\right)_{c}=\frac{1}{\sqrt{2 L}} \sum_{n=\frac{1}{2}, \frac{3}{2} \ldots}^{\infty}\left(b_{n, c} e^{-i \frac{n \pi}{L} x^{-}}+d_{n, c}^{\dagger} e^{i \frac{n \pi}{L} x^{-}}\right)
$$

and assigning the relations

$$
\left\{b_{n, c_{1}}, b_{m}^{\dagger c_{2}}\right\}=\left\{d_{n}^{c_{1}}, d_{m, c_{2}}^{\dagger}\right\}=\delta_{c_{2}}^{c_{2}} \varepsilon_{n, m}
$$

to the coefficients. It has proven useful to maintain the distinction between upper and lower color indices, as in these relations, particularly in the computer code. The adjoint representation, however, is real and in it no such distinction exists.

To treat this system numerically: it is quantized in a box in $x^{-}$of length $2 L$. Because fermionic fields appear in measurable quantities as bilinears. either poriodic or antiperiodic boundary conditions are appropriate. As previously discussed. the latter will be employed to allow for an expansion in solutions of the free wave equation which is also complete.

The Fock space of states is constructed by applying creation operators 10 ithe vacuum in all possible independent color-singlet combinations. The operatur: $p^{-}$ and $P^{-}$in this basis are obtained by substituting the expansion in Eq. (1.nit intw, Eqs. (1.82) and (1.83). 
The result is, for $P^{+}$,

$$
P^{+}=\left(\frac{2 \pi}{L}\right) \sum_{n=\frac{1}{2}, \frac{3}{2}, \ldots}^{\infty} n\left(b_{n}^{\dagger c} b_{n, c}+d_{n, c}^{\dagger} d_{n}^{c}\right)
$$

after discarding an infinite vacuum momentum. $P^{+}$is diagonal in this basis. asci independent of both quark mass $m$ and coupling $g$. Quarks carry a momentum $2 \pi n / L$ in physical units. That $P^{+}$generates translations in $x^{-}$may be expicicity verified:

$$
\left[P^{+}, i \cdot R\left(0, x^{-}\right)\right]=-i \partial^{+} L_{R}=-i 2 \partial_{-} \iota_{R}
$$

The charge operator $Q^{a}$ defined in terms of the normal-ordered current.

$$
Q^{a}=\int_{-L}^{L} d x^{-}: L_{R}^{\dagger} T^{a} \xi_{R}:=\sum_{n=\frac{1}{2}, \frac{3}{2}, \ldots}^{\infty}\left(b_{n}^{\dagger} T^{a} b_{n}-d_{n}^{\dagger} T^{a} d_{n}\right)
$$

is also diagona!. It generates global color transformations.

$$
\left\{Q^{a} \cdot 2 \cdot R \cdot L\right\}=-T^{a}{ }^{a} \cdot R \cdot L
$$

and

$$
\left[Q^{a}, A^{-b}\right]=\imath f^{a b c} A^{-c}
$$

witit. 2.2 and $A^{-a}$ deficed as functions of $i \cdot R$

Finally, $P^{-}$is relatively complicated. is dependent on the coupling culsoint. ard acts as the Hamiltonian. evolving the system in $I^{-}$, In two dimerusini : caryes the climension of mass. The quark mass $m$ is the only other dimesn...... 
parameter, and apart from an overall and arbitrary mass scale, solutions depend on the ratio $\mathrm{g} / \mathrm{m}$. It is convenient to introduce the dimensionless coupling constaut

$$
\lambda \equiv\left(\frac{1}{1+\pi m^{2} / g^{2}}\right)^{\frac{1}{2}}, \quad \text { or } \quad \frac{g}{m}=\left(\frac{\pi \lambda^{2}}{1-\lambda^{2}}\right)^{\frac{1}{2}} .
$$

as in Ref. [20], where $\lambda$ varies from zero when $g / m$ is zero, to one when $g i m$ is infinite.

Defining the dimensionless momentum

$$
K \equiv\left(\frac{L}{2 \pi}\right) P^{+}
$$

and Hamiltonian

$$
H \equiv\left(\frac{2 \pi}{L}\right) \frac{\pi^{2} \lambda^{2}}{g^{2}} P^{-}=\left(\frac{2 \pi}{L}\right) \frac{\left(1-\lambda^{2}\right)}{m^{2}} P^{-},
$$

the dependence on the box length drops out of the mass- squared operator, and

$$
M^{2}=P^{+} P^{-}=\frac{g^{2}}{\pi \lambda^{2}} K H=\frac{m^{2}}{\left(1-\lambda^{2}\right)} K H
$$

The dimensionless Hamiltonian $H$ separates into the free and interacting terms. $H_{0}$ and $H_{I}$, with

$$
H=\left(1-\lambda^{2}\right) H_{0}+\lambda^{2} H_{l}
$$

The free Hanilunian $H_{0}$ sums the energ: $\mathrm{m}^{2} / \mathrm{h}^{+}$of free qua:ks.

$$
H_{0}=\sum_{n=\frac{1}{2}, \frac{3}{2} \ldots,}^{\infty} \frac{1}{n}\left(b_{n}^{\dagger c} b_{n, c}+d_{n, c}^{\dagger} d_{n}^{c}\right) .
$$

while the terms in $H$, are displayed in Tables [1] and [ 2 ". 
Table [1]

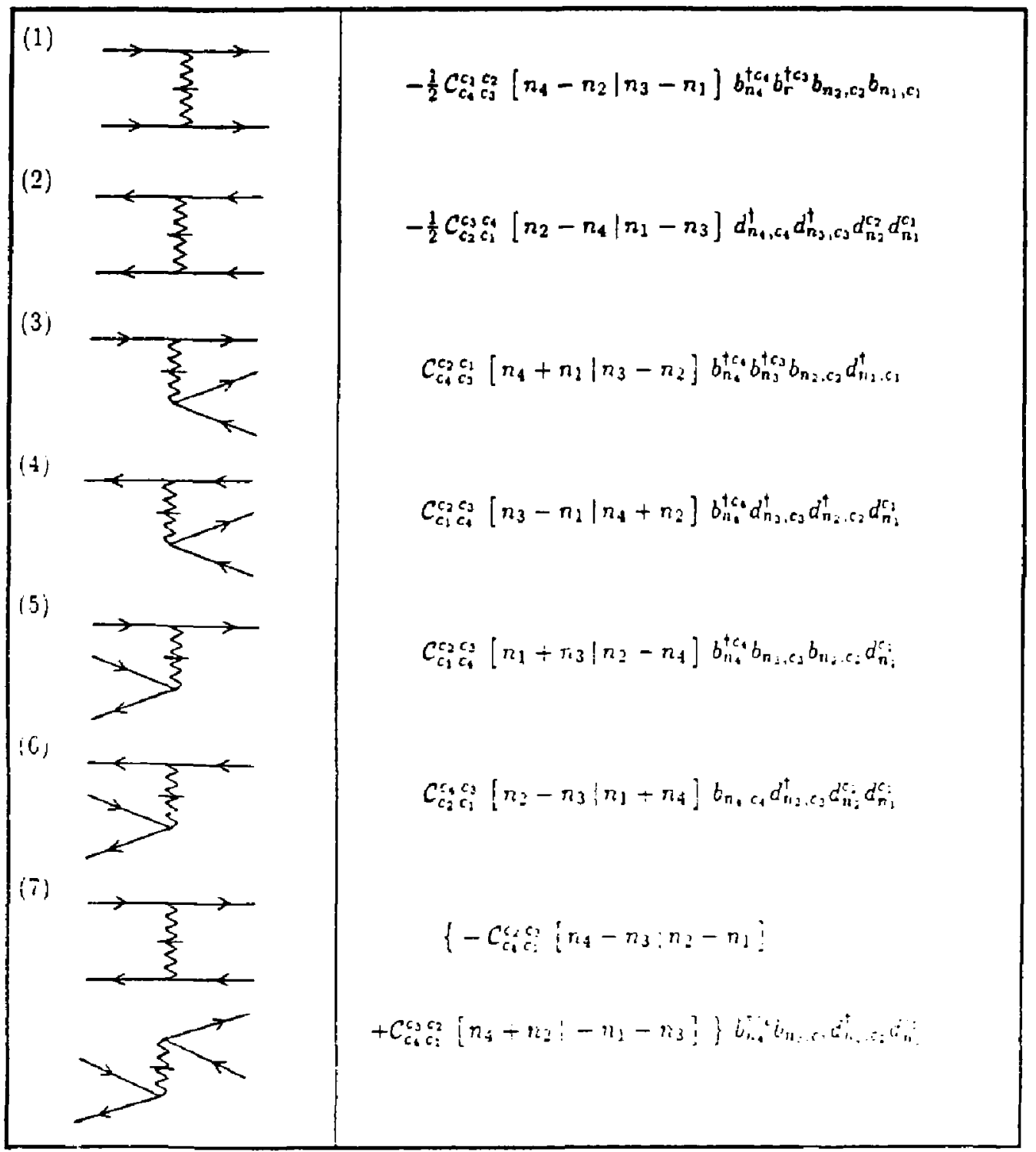


Table [2]

(S)

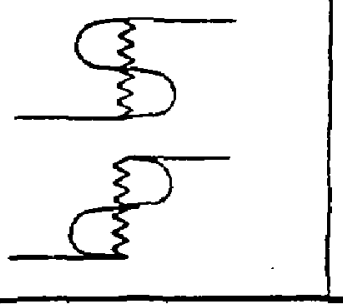

$$
\begin{aligned}
& \frac{3}{2}\left(\frac{N^{3}-1}{2 N}\right) \sum_{\ell}\{[n-\ell \mid \ell-n] \\
& \quad-[n+\ell \mid-n-\ell]\}\left\{b_{n}^{\dagger c} b_{n, c}+d_{n, c}^{\dagger} d_{n}^{c}\right\}
\end{aligned}
$$

These are summed over the half-integers $n_{1}$ such that momentum is conserved. The color matrix is defined by

$$
\mathcal{C}_{c_{2} c_{1}}^{c_{1} c_{3}} \equiv\left[T^{a}\right]_{c_{4}}^{c_{1}}\left[T^{a_{j}}\right]_{c_{2}}^{c_{3}}=\frac{1}{2 N}\left(N \delta_{c_{2}}^{c_{1}} \delta_{c_{4}}^{c_{3}}-\delta_{c_{2}}^{c_{1}} \delta_{c_{4}}^{c_{1}}\right)
$$

while the brachet

$$
[n \mid m] \equiv \frac{1}{n^{2}} \delta_{n+m, 0}
$$

represents the instantaneous gluon propagator corresponding to the linear putent jal in $I^{-}$.

The interaction Haniltonian $H_{l}$ is composed of a product of currents $j^{+a}$ which are individually normal-ordered. Rearranging terms to put $H_{I}$ in norma! order, as in Table i1), produces the quark self-energy terms of Table $\{2 ;$. Thesc interactions include a finite renormalization of the quark mass. and. in color singlets. cancel infrared divergences from the four-quark interactions.

The possibility of infrared divergences due to the divergence of the gluon prui-

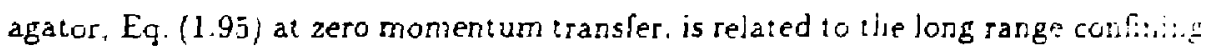
beliavjor of the linear potential. Eq. (1.85) is onl $\because$ sinc:! correct for non-z!: $:$ 
and $m$. The finite box length naturally regulates this beharior, and the contribu. tion to the interacting part of $P^{-}$when either $n$ or $m$ or both are zero may be computed directly form Eq. (1.83). It gives

$$
-\frac{g^{2}}{8}\left[\frac{1}{L}\left(Q^{a} Q^{a(2)}+Q^{a(2)} Q^{a}\right)+L Q^{a} Q^{a}\right]
$$

This vanishes between color singlets, and there are therefore no infrared divergences in this sector. It is consistent, then, to simply discard interactions with zero momentunz transferred.

In the continuum. several prescriptions have been employed to regulate the gluon propagator, including a sharp cutoff in the momentum transfer $p^{+}$near zero ${ }^{[2.22]}$ and pole prescriptions for $1 / p^{2}$ such as ${ }^{[23 !}$

$$
\frac{1}{2}\left(\frac{1}{\left(p^{+}\right)^{2}+i t}+\frac{1}{\left(p^{+}\right)^{2}-i \epsilon}\right)
$$

and

$$
\frac{1}{2}\left(\frac{1}{\left(p^{+}+i \epsilon\right)^{2}}+\frac{1}{\left(p^{+}-i \epsilon\right)^{2}}\right) .
$$

The differences are simplest to understand in terms of the spat:al potentials that their Fourier transforms produce. The third is simply $\left|x^{-}\right|$, while the first two effectively shift the potential by an infinite and negative constant. As is clear foraz Eq. (1.83). such a shift adds a term to $P^{-}$proportional to $\left(f^{2} ; Q^{u} Q^{\prime \prime}\right.$. which for the system in a box, is equivalent to including the term $\left(g^{2} / 4\right) L Q^{2} Q^{a}$. which appears in $P^{-}$directly in the prtential. This makes confinement manifes: in both the potential and in quark propagators, and in Reis. [21] and [2], was unced 
to calculational adrantage. In the space of color singlets, however, the various prescriptions are consistent.

As a final comment on the Hamiltonian, no terms appear in which quarks either disappear or emerge from the vacuum. This is a consequence of the combination of the positive-definiteness of the light-cone momenta and the requirement of its conservation at vertices. In contrast to equal-timequantization, the vacuum cannot be coupled to any other state, and so is an exact eigenstate.

The Lorent $z$ boost generator in terms of only $R$ fieids.

$$
h_{B}=\frac{1}{2} \int_{-L}^{L} d r^{-} x^{-} \psi_{R}^{\dagger} i \vec{\partial}_{-} h,
$$

after discretization becomes

$$
\begin{aligned}
h_{B}=\frac{-3}{2} \sum_{m, n=\frac{1}{2}, \frac{3}{2}, \cdots} & {\left[(-1)^{2(n-n)} \frac{(n+m+1)}{m-n}\left(b_{m}^{\dagger} b_{n}-d_{n}^{\dagger} d_{m}\right)\right.} \\
& \left.+(-1)^{2(m+n+1)} \frac{(m-n)}{m+n+1}\left(b_{m}^{\dagger} d_{12}^{\dagger}+d_{m} b_{n}\right)\right]
\end{aligned}
$$

where the divergent term is discarded when $m=n$. As may be verified. at $x^{+}=0$. $\psi_{R}$ obeys the boost relation appropriate for a right-handed. spin one-half fie.t.

$$
\left.i\left[K_{B}, \imath_{R}\left(x^{-}\right)\right]=\left(\frac{1}{2}+x^{-} \partial_{-}\right) 2 \cdot R^{\left(x^{-}\right.}\right)
$$

even at finite $L$. Fields which depend upon $\tau^{\prime} R$, such as $4 L$, will also transtorm cor. rectly, apart from boundary terms. which kould require the retention of bouldiry fields in Eq. (1. $19 \%$. 
Lorentz invariance is broken by choosing boundary conditions at a fixed $L_{\text {; }}$ while not evident in Eq. (1.101), jt can be seen in that $k_{B}$ is not diagonal, and so does not annihilate the vacuum. In the continuum limit, however, the non-diagonal term oscillates to zero, and the Lorentz invariabce of the vacuum is restored.

\subsection{LORENTZ AND PARITY PROPERTIES OF IVAVEFUNCTIONS}

The effect of Lorentz boosts and parity transformations on wavefunctions is simp'est to study in the continuum, where it is convenient to normalize the creation and annihilation operators relativistically! $!^{(24)}$ For example.

$$
\left\{b_{p^{+}, c_{1}}, b_{k^{+}}^{\dagger c_{2}}\right\}=4 \pi k^{+} \delta_{c_{2}}^{c_{2}} \delta\left(k^{+}-p^{+}\right)
$$

and so forth. With this normalization,

$$
v_{R}\left(z^{-}\right)_{c}=\int_{0}^{\infty} \frac{d p^{+}}{4 \pi p^{+\frac{1}{2}}}\left[b_{p^{+}, c} e^{-\frac{1}{2} p^{+} z^{-}}+d_{p r}^{\dagger} \epsilon^{\frac{1}{2} p^{+} z^{-}}\right]
$$

so that ${ }_{R}$ satisfies

$$
\left.\left\{u^{*} R(z)_{c_{1}}, \psi_{i}^{\dagger}(y)^{c_{z}}\right\}_{z^{+}=y^{+}}=\delta_{c_{1}}^{c_{2}} \delta z^{-}-y^{-}\right) .
$$

Here, $z$ is employed as a spacetime variable to avoid confusion with the nomentitu: fraction. or Bjorken variable, $\boldsymbol{x}$. Also.

$$
Z_{L}\left(z^{-}\right)_{c}=\int_{0}^{\infty} \frac{d p^{+}}{4 \pi p^{+\frac{1}{2}}}\left(\frac{m}{p^{+}}\right)\left[b_{p^{+}, c} \epsilon^{-\frac{1}{2} p^{+} z^{-}}-d_{p c}^{\dagger} \epsilon^{\frac{1}{2} p^{+} z^{-}}\right] .
$$


Eigenstates may be normalized analogously,

$$
\left\langle\phi\left(p^{+}\right) \mid \varphi\left(k^{+}\right)\right\rangle=4 \pi k^{+} \delta\left(k^{+}-p^{+}\right) .
$$

Then, for example, if the valence meson is defined such that

$$
\left|\phi\left(p^{+}\right)\right\rangle=\int_{0}^{1} \frac{d x \phi(x)}{\left[4 \pi N^{+} x(1-x)\right]^{\frac{1}{2}}} U_{(1-x)^{+}}^{t c} d_{x p^{+}, c}^{t}|0\rangle
$$

the wavefunction $o(x)$ is normalized to one:

$$
\int_{0}^{1} d x \phi^{2}(x)=1
$$

This normalization is convenient, as it matches that of the wavefunctions from the program.

The generator of Loreriz boosts at $:^{+}=0$,

$$
K_{B}=\frac{1}{4} \int_{-\infty}^{\infty} d z^{-} z^{-} \Theta^{++}=\frac{1}{2} \int_{-\infty}^{\infty} d z^{-} z^{-}+t_{R}^{\dagger}+\vec{\partial}_{-} i_{R}
$$

is then

$$
\frac{i}{8 \pi} \int_{0}^{\infty} d p^{+}\left[\left(\partial_{p^{+}} b_{p^{+}}^{\dagger c}\right) b_{F^{+}, c}-b_{p^{+}}^{\dagger c} \partial_{p^{+}} b_{p^{+}, c}+\left(\partial_{p^{*}} d_{p^{+}, c}^{\dagger}\right) d_{p^{+}}^{c}-d_{p^{+}, c}^{\dagger} \partial_{p^{+}} d_{p^{+}}^{c}\right] . \quad(1.110
$$

As a result.

$$
\left[i_{B}, b_{p^{+}}^{\dagger c}\right]=t p^{-} \partial_{p^{+}}+b_{p^{+}}^{\dagger c} .
$$


and after some algebra,

$$
K_{B}\left|\phi\left(p^{+}\right)\right\rangle=i p^{+} \partial_{p^{+}}\left|\phi\left(p^{+}\right)\right\rangle
$$

The corresponding finite transformation is then

$$
e^{s a K^{\prime}}\left|\phi\left(p^{+}\right)\right\rangle=\left|\phi\left(e^{-\alpha} p^{+}\right)\right\rangle
$$

boosting $\left|\phi\left(p^{+}\right)\right\rangle$only scales the total momentum $p^{+}$. The fraction $x_{n}$ of $p^{+}$carried by each constituent remains unchanged, so that the wavefunction $\phi(x)$ is boostinvariant.

While Lorentz invariance is greatly sinplifed by quantizing on the light cone. parity becomes less transparent. In two dimensions, there are only longitudira] boosts, and since these leave the quantization surface intact, it is possible to construct a Hamiltonian, $P^{+} P^{-}$, and therefore wavefunctions, which are joost invariant. On the other hand, parity $\left(x^{1} \leftarrow-x^{1}\right)$ interchanges $x^{+}$and $x^{-}$, and $P^{+}$ and $P^{-}$, so the formalism treats left and right unevenly. Severtheless, while parity. interchanges $P^{+}$and $P^{-}$, it clearly leaves the operator $M P^{2}=P^{+} P^{-}$unchanged. It is therefore possible, and when non-degenerate necessary for states to have definite parity.

To see how the parity operation affects wavefunction coordinates. conside: a state of $n$ particles of equal mass $m$, each carrying momentum fraction $r_{1}=$ $k_{1}^{+} / P^{+}=\left(k_{2}^{0}+k_{1}^{1}\right) / P^{+}$and $P^{+}=\sum_{1} k_{1}^{+}$. Define $\tilde{x}_{1}$ to be the corresponding momentum fractions of the parity transformed state. To solve for these, expluit tive fact that the $x_{1}$ are boost invariant to work in the center of inass frame so tha: 
$\sum k_{i}^{1}=0$. This will also be true in the transformed state where $k_{i}^{1} \rightarrow-k_{i}^{1}$. As a result,

$$
P^{+}=\sum_{i}\left(k_{i}^{0}+k_{i}^{1}\right)=\sum_{i} k_{i}^{0}
$$

in both systems. Then

$$
x_{i}=\frac{k_{1}^{0}+k_{i}^{1}}{P^{+}} . \quad \tilde{x}_{1}=\frac{k_{1}^{0}-k_{1}^{1}}{P^{+}}
$$

and

$$
x_{1} \dot{x}_{1}=I_{2} \dot{x}_{2}=\cdots=I_{n} \tilde{x}_{n}=\frac{m^{2}}{P+2} .
$$

Combined with

$$
\sum_{i} \dot{f}_{1}=\sum_{i} \dot{x}_{i}=1
$$

this yields

$$
\tilde{x}_{1}=\frac{1}{I_{1}}\left(\frac{1}{\sum, \frac{1}{x_{1}}}\right) .
$$

For the particular case of two equal mass particles, the momentum fractions $x$ and $1-x$ are simply interchanged. This inverse reiation can be understuoul by noting that by $I=\left(k^{0}+h^{1}\right) / P^{p+}$ fart left-mmers have $I$ near zero as $-k^{1}$ 
approaches $k^{0}$, fast right-movers have $x$ near one, and parity interchanges left and right.

Finally note that discretization by setting boundary conditions at $\pm L$ on the $z^{-}$axis alone expljcitly breaks parity. The $x_{i}$ are restricted to a particular set of integers djvided by the total integral momentum. Eqn. (1.118) then maties parity breaking evident, as the $\overline{\boldsymbol{x}}_{\boldsymbol{i}}$ will not in general be contained in this set. Obviously, this is remedied in the continuum limit. For the special case of two particles, however, it is soluble for any box size, since the $x$ and $l-x$ which are interchanged are already defined. Consequently, to determine the parity for a meson wavefunction, for example, it is only necessary to examire the minimum Fock ( $q \bar{q})$ wavefunction, at any $K$, for symmetry under momentum interchange. For example, the lowest mass eigenstates for any $N$ or coupling are of the form

$$
\int_{0}^{1} \frac{d x \varphi(x)}{(4 \pi V x(1-x))^{\frac{3}{2}}} b_{(1-x) p^{\dagger}}^{\dagger c} d_{x p^{+}, c}^{\dagger}|0\rangle
$$

with $\phi$ symmetric under interchange of $x$ and $1-x$. Interchanging these in the creation operators and accounting for the opposing signs for particles and antiparticles under parity produces an over-all minus sign and shows this to be a pieudoscalar. An odd $\varphi(x)$ represents a scalar.

Alternatively; parity may be determined by computing the vacuum io one nie. son matrix elements of the scalar and pseudoscalar color singlet operators $i^{-} \cdot$ and $\bar{\psi} \gamma^{5} \psi^{122 !}$ These may be evaluated directly in terms of fields and the $q \bar{q}$ compunemut of the meson wavefunction: 


$$
\left\langle 0|: \bar{\psi} \psi(z):| \phi\left(k^{+}\right)\right\rangle=-m N^{\frac{2}{3}} e^{-i k z} \int_{0}^{1} \frac{d x \phi(x)}{(4 \pi)^{\frac{1}{2}}}\left(\frac{1}{x}-\frac{1}{1-x}\right)
$$

and

$$
\left\langle 0\left|: \bar{\psi} \gamma^{5} \psi(z):\right| \dot{\phi}\left(k^{+}\right)\right\rangle=-m N^{\frac{1}{3}} e^{-i k z} \int_{0}^{1} \frac{d x \phi(x)}{(4 \pi)^{\frac{1}{2}}}\left(\frac{1}{x}+\frac{1}{1-x}\right)
$$

As expected, a $\phi(x)$ odd under $x \mapsto l-I$ is a scalar and couples $\pi l y$ to $-2 \cdot$ while an even $\phi(x)$ is a pseudoscalar.

Finally, charge conjugation on t'iese $q \bar{q}$ components interchanges quark and antjquark. Permuting operators and redefining morientum variables gives the same sign as parity, and all meson states are charge-parity even.

\subsection{CHIRAL ANOMALY}

The interacting part of the Hamiltonian $P^{-}$is expressed entirely as a function of the current

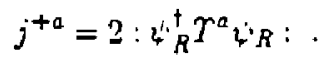

The commutator of $j^{+a}$ with itself is relatively easy to compute, and the erointicu of $j^{+n}$ due to the interaction follows immediately. This allows for a quick and dirty calculation of the non-Abelian axial anomaly. assuming only that the gaugr curreat is conserved. 
The axial current

$$
\begin{aligned}
& j_{5}^{+a}=2 \psi_{R}^{\dagger} T^{a} \psi_{R} \\
& j_{5}^{-a}=-2 \psi_{L}^{\dagger} T^{a} \psi_{L},
\end{aligned}
$$

differs from the vector current

$$
\begin{aligned}
& j^{+a}=2 j_{R}^{\dagger} T^{a} \psi_{R}^{\prime} \\
& j^{-a}=2 \psi_{L}^{\dagger} T^{a} \psi_{L}-f^{a b c} \partial_{-} A^{-b} A^{-c},
\end{aligned}
$$

in the $j^{-a}$ term,

$$
j_{5}^{-a}=-j^{-a}-f^{a b c} \partial_{-} A^{-b} A^{-c} .
$$

The gauge fields should be considered as appropriately symmetrized, and the currents as normal-ordered. If the vector current is conserved, then

$$
\partial_{-} j^{-a}=-\partial_{+} j^{+a}
$$

and therefore

$$
\partial_{\mu} j_{5}^{\mu a}=2 \partial_{+} j^{+a}-f^{a b c} \partial_{-}^{2} A^{-b} \cdot A^{-c}
$$

By the constraint equation

$$
-\partial_{-}^{2} A^{-b}=\frac{2}{2} j^{+b}
$$

for $A^{-b}$,

$$
\partial_{\mu} j_{5}^{\mu a}=2 \partial_{+} j^{+a}-f^{a b c} A^{-\grave{b}} j^{+c} .
$$

Because the anomalous term depends only on the interaction. only that part of $P^{-}$is needed to compute $\partial_{+} j^{+a}$, and the tern in $P^{-}$proportional to $m$ wili be neglectes. 
The commutators involving $j^{+a}$ are defined by first point splitting the two $\psi_{R}$ factors in $j^{+a}$ a distance $\epsilon$ along $x^{-}$. The exponential in $A^{+a}$ needed to preserve gauge invariance is absent due to the choice of gauge. Then, using the expansion in Eq. (1.84), at $x^{+}=0$

$$
\begin{aligned}
& {\left[j^{+a}\left(x^{-}\right), j^{+b}\left(y^{-}\right)\right]=2 i f^{a b c} j^{+c}\left(x^{-}\right) \varepsilon\left(x^{-}-y^{-}\right)} \\
& -\frac{i \delta^{a b}}{2 L \sin (\pi \epsilon / L)}\left[\delta\left(x^{-}-y^{-}-c\right)-\delta\left(x^{-}-y^{-}+\epsilon\right)\right] .
\end{aligned}
$$

As $t$ vanishes, the second (Schwinger) term on the right may be represented in the more conventional form

$$
\frac{i}{\pi} \delta^{a b} \partial_{x^{-}} \delta\left(x^{-}-y^{-}\right)
$$

After some algebra and to leading order in $\epsilon$.

$$
\begin{aligned}
& 2 \partial_{+} j^{r a}\left(x^{-}\right)=i\left[P^{-}, j^{+a}\left(x^{-}\right)\right]= \\
&-\frac{g^{2}}{\delta \pi} \int_{-L}^{L} d y^{-} \epsilon\left(x^{-}-y^{-}\right) j^{+a}\left(y^{-}\right) \\
&-\frac{g^{2}}{4} \int_{-L}^{L} d y^{-}\left|x^{-}-y^{-}\right| j^{+b}\left(y^{-}\right) j^{+c}\left(x^{-}\right) .
\end{aligned}
$$

The constraint equations for $A^{-a}$ and $\partial_{-} A^{-a}$, neglecting bourdary terms and using

$$
\partial_{-} A^{-a}=\frac{1}{2} \epsilon_{\mu \nu} F^{\mu \nu^{\alpha}}
$$

produces

$$
2 \partial_{+} j^{+a}\left(x^{+}\right)=\frac{g}{4 \pi} \cdot{ }_{\mu \nu} \Gamma^{\mu \nu a}+g f^{a b c} \cdot A^{-b} j^{+c} .
$$


This may be rewritten as

$$
D_{+} j^{+a}=\frac{g}{4 \pi} \epsilon_{\mu \nu} F^{\mu+}
$$

using

$$
D_{+}^{a c}=\partial_{+} \delta^{a c}-\frac{1}{2} f^{a b c} A^{-b}
$$

also, in this gauge,

$$
D_{-}^{a c}=\partial_{-} \delta^{a c} .
$$

Finally, combining Eqs. (1.12i) and (1.135),

$$
D_{\mu j}^{\mu}=\frac{g}{4 \pi} \epsilon_{\mu \nu} F^{\mu \nu \alpha}
$$

This result is referred to as the covariant anomaly, and is discussed in Ref. [25].

The discussion in this work will be conducted predominantly in momentum space, and it is convenient to bave a version of the algebra of Eq. (1.130) in terms of the transformed operators

$$
V_{k}^{a}=\frac{1}{2} \int_{-L}^{L} d x^{-} e^{-i \frac{k L}{L} x^{-}} j^{+a}\left(x^{-}\right) .
$$

The fermion fields satisfy antiperiodic boundary conditions, but the currest, which is a product of these, satisfies periodic conditions, and the monentum $k$ in this exflansion is then an integer. By Eq. (1.130), these operators satisfy the liacMood: algebra ${ }^{[26]}$

$$
\left[V_{k}^{a}, V_{\ell}^{b}\right]=i f^{a b c} V_{k+\ell}^{c}+\frac{1}{2} \varepsilon \delta^{a b} \varepsilon_{k+l .0} .
$$

Because states are restricted to color singlets, the trans'orm of the $C^{*}(1)$ current will be particularly important, as will be discussed in Chapler $\{\hat{\jmath}\}$. 


\section{NUMERICAL RESLLTS}

\subsection{OVERVIE* OF PROGRAM}

The momentum, charge and Hamiltonian operators have been expressed as the dimensionless functions $K, Q$ and $H$ of a discrete and denumerable Fock basis. $K$ is already diagonal, so that it only remains to diagonalize $H$ in a space in which $Q$ vanishes to determine the masses and wavefunctions of this system. This is best done by computer, and this section gives a brief outline the program used to accomplish this. This was run on an IBM 30S1, with CPL time for the cases 10 be presented typically in the range of a few minutes.

Once $N, B$ and $2 K$ are specified, all possible color-singlet combinations of $\gamma$. colozed quarks consistent witb the conserved quantities $B$ and $2 K$ are gent. zted. (The momentum $K$ is the sum of half-integer quark momenta, so that it is convenient to use $2 K$, which is always an integer, in this discussion.) In particular. to satisfy baryon number, all states begin with $B \Lambda^{\prime}$-tuplets of quarks. ( $B$ is re. stricted to be zero or positive, which is sufficiently general by charge conjugation invariance.) These quarks are understood to be contracted into $S(i)$ singlets by antisymmetrizing in color, although in practice it is not necessary to explicitly. introduce color indices in order to compute color factors. To these baryons are appended quark- antiquark $(q \bar{q})$ pairs. from zero to the maximum permitted by the momentum $2 K$ which is divided into the positive-definite monenta carried by individual quarks. Again, these mesons are contracted into singlets. As for the baryons, color is fully contracted, and it is enough to know only which quarks at. grouped into whi=t. singlet. 
In addition to mesons, baryon-antibaryon pairs could be added; however, these are redundant to states formed by adding mesons. Finally, for $U^{\prime}(N), B$ is required to be zero, as baryon number is essentially an electric charge for the extra [111 and is also confined.

The program to construct the Fock space is built around two subroutines. The first generates all possible combinations of $n$ integers such that they sum to a fixed number, but with a particular integer limited to at most $i$ appearances in each permutation. This is used to subdivide a fixed momentum among various particles. The index $i$ accounts for Fermi statistics; for example, $i=\Lambda$ for quarks (with one flavor) because the color degree of freedom allow's the same momentum to be carried by at most $N$ quarks. These permutations are stored and their locations recorded. The second routine is similar, except that rather than permuting momenta, it generates permutations of the locations of the entire momentum distributions previously stored. The result has something of a tree structure. For example, for a subset of states with three mesons and two baryons, the possible ways of sp'itting momentum between mesons and baryons are run through. Within this, the total momentum of the mesons is split among the three mesons with bosonic statistics: the rest is split between the baryons with fermionic or bosonic statistics depending on $N$ being odd or even. Once assigned, the possible nomenta within the individual mesons or baryons are iterated through. The fina! momentum assignment tw individual guarks is checked to ensure that no more than $x$ are the sarne befur: the state is stored.

The resulting Fock space is in general not orthonormal and frequently on: . complete. To remove redundan: states, the inner-product matrix is conin: 
and diagonalized, in the same way as the Hamiltonian is later computed. Overcompleteness is indicated by zero eigenvalues. Appropriate states are dropped, the new matrix is again diagonalized, and so on, until no zero eigenvalues remain. The resulting matrix of eigenvectors with the eigenvalues divided out orthonormalizes the final set of states. Finally, options to further restrict the states by limiting the total number particles in each state by band, or by discarding those whose invariant mass exceeds a cutoff, are included.

The operators in the individual terms in the Hamiltonian are represented in the same form as the Fock states. The Hamiltonian matrix is evaluated by sandwiching these between the complete but not orthonormal states. and then iterati:g through the momenta which these operators could carry. The original states are used in this stage because they are in general simpler to represent. As elaborated in the Appendix $A$, these inatrix elements are then rort racted and the gluon propagators and color factors are computed. Finally, the resulting Hamiltonian matrix is converted to the corresponding matrix in the orthonormal basis.

Finally, the Hamiltonian is diagonalized by standard, pachaged routines. using LU decomposition; see, for example, Ref. [2 $\pi$ ]. This produces both the fu!! spectrum of states and the corresponding normalized wavefunctions, from which any computable quantity can be extracted up to the resolution $K$. Typical valus for $K$ used here were between 5 and 10, and in these cases, the time required to diagonalize the Hamiltonian was insignificant relative to that needed to evaluat: it.

Because the Hamilionian $m^{2} H_{0}+g^{2} H_{l}$ breabs up into a free and interartiog part such that the parameters $m^{2}$ and $g^{2}$ appear as overall constarts before ecel... 
the matrices $H_{0}$ and $H_{J}$ are stored separately. Computing the spectrum for different $g$ or $m$ then only involves computing $H_{0}$ and $H_{l}$ once, and then recombining and rediagonalizing at eacl $g$ and $m$, with effectively no extra cost in time.

\subsection{SPECTRA}

One of the advantages of directly diagonalizing the Hamiltonian is that it produces information about the entire spectrum of the theory in the form of eigenvalLes (masses) and eigenfunctions (wavefunctions), rather than of only the lightest states.

Figs. (2) and (3) display the development of the $1+1$ dimensional spectra for three colors, with baryon number of zero and one at relatively weak ( $\lambda=.3325$ or - $m / g=1.6)$ and relatively strong $(\lambda=.9847$ or $\mathrm{m} / \mathrm{g}=.1)$ coupling.

As is evident, the number of states is limited by the total momentum $\Pi^{\circ}$. As $K$ increases and the continuum limit is appronched, Figs. (2a) and (2b) show the development of both a discrete set of bound states at low mass and a continuum of states with a threshold at $4 . I^{\prime 2}$, with $M$ the mass of the lighest meson or baryon. It is clear that these states are both filling in the continuum with increasing $K$, and also maintaining some structure.

At a particular $K$, the states in the spectrum are eigenstates of buth $P^{+}$and $\mathrm{P}^{-}$: in particular, individual states carnot decay. Inform rion abou! resonantes. for example. must be contained within this structure.

For the case with strong coupling. Figs. $\left\{3 a_{j}\right)$ and $\{3 b$, a large number of sta:es ciuster near zero mazs. above which sets a teiaively large gap. In the 


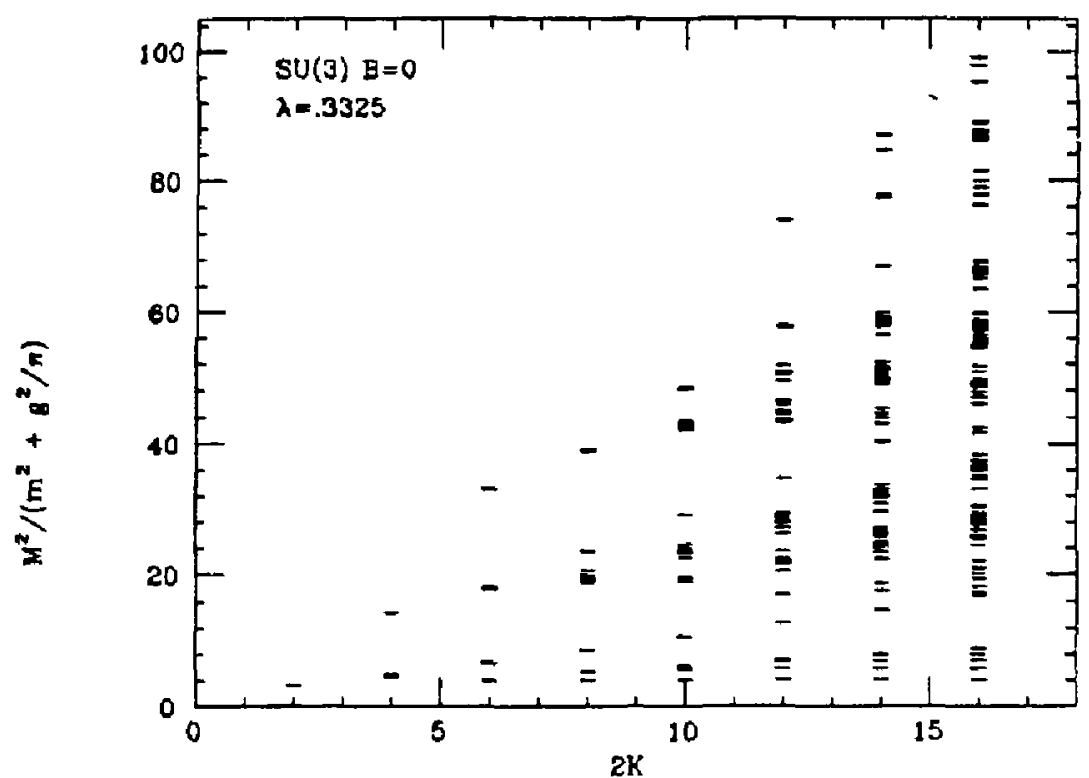

(a)

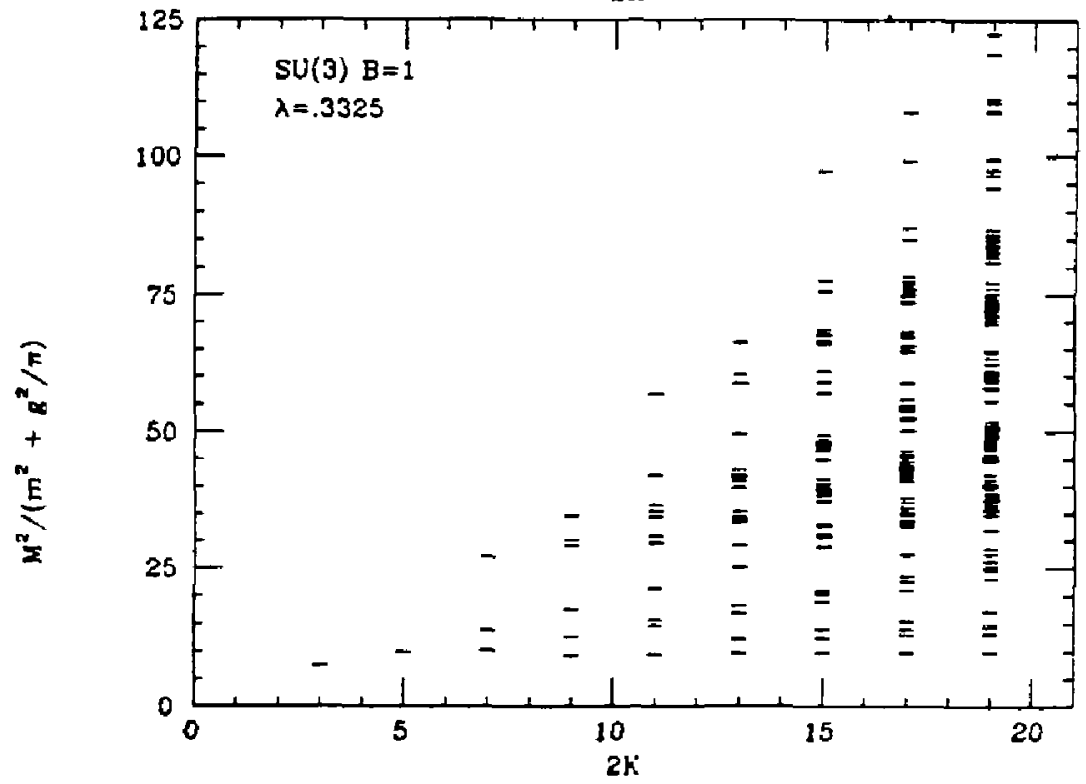

(b)

Figure 2. Development of $N=3$ Spectrun with Increasing $N$ : lleán Coupling (a) Mtra. Spectum $\{B=0$ ) (b) Baryon Spectrum $(B=1$ ) 

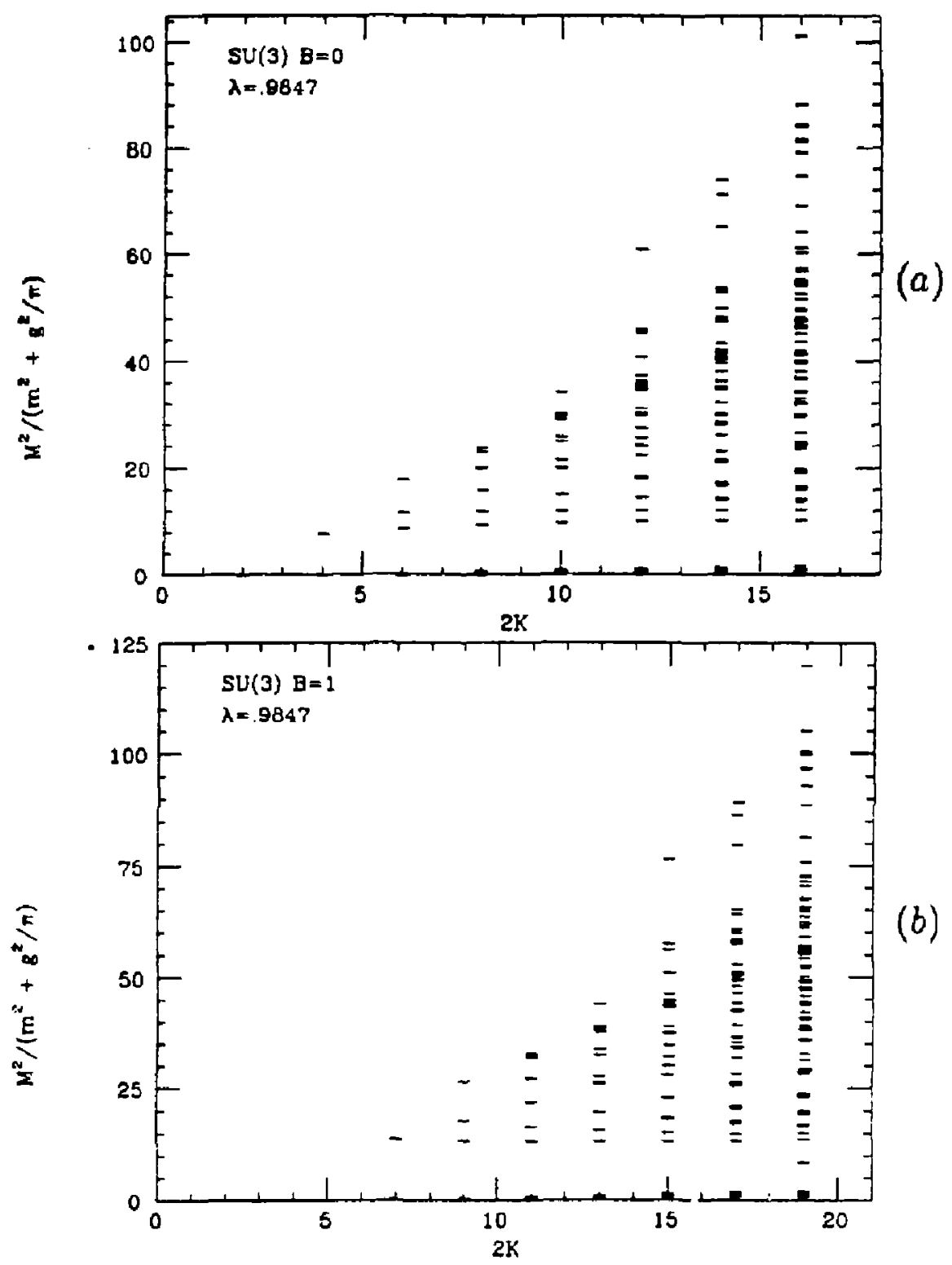

Figure 3. Development of Spectrum with Increasing $\ddot{n}$. Strong Couphing (a) Meson Sprerit..t. $(B=0$, (b) Baryen Spectum $(B=1)$. 
continuum limit, the number of states in this cluster will become infinite, generating a continuum of levels which begins near zero.

That this method produces an entire spectrum is a great advantage, but it can also have its drawbacks. In particular, for very large $K$ the number of eigenstates can become enormous. The higher states will in general contain both new, excited states as well as states with multiple copies of lighter particles with some relative momentum. Developing methods to extract those of interest will become particularly important in four dimensions, due to the large number of degrees of freedom. In a sense, being able to control the number of states by leeping $R$ finite becomes an advantage. Restricting states by means of a Lorentz-invariant cutoff. which will be introduced later, may be even more useful, as a continuum limit may be taken while a fixed cutoff is imposed. Finally, it will be essential in four dimensions to take advantage of all the residual spacetime and internal symmetries in the light-cone Hamiltonian to separate the Fock space into disjoint sectors, as was done with baryon number in these cases.

In Figs. ( $\mathrm{a}-\mathrm{c})$, the meson $(B=0)$, single baryon $(B=1)$, and double baryon $(B=2)$ spectra are traced from zero coupling (or infinite quark mass) at $\lambda=0$ to infinite coupling (zero mass) at $\lambda=1$ with $K$ held fixed. The dimension!ess coupling $\lambda$, as well as $M^{2}$ in units of $m^{2}+g^{2} / \pi$ are quantities emploved directly in the program. For each of these plots, the Hamiltonian mairices $H_{0}$ and $H_{I}$ are computed once and stored. At each value of $\lambda$ (or $\mathrm{m} / \mathrm{g}$ ), these are acided to form the full Hamiltonian

$$
H=m^{2} H_{0}+g^{2} H_{l}
$$




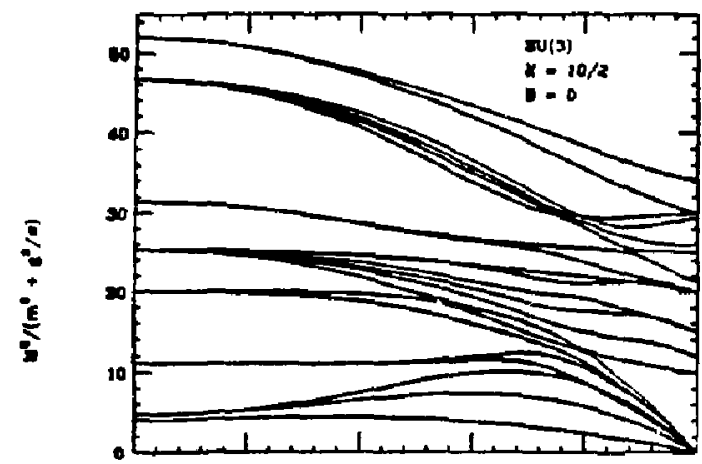

(a)

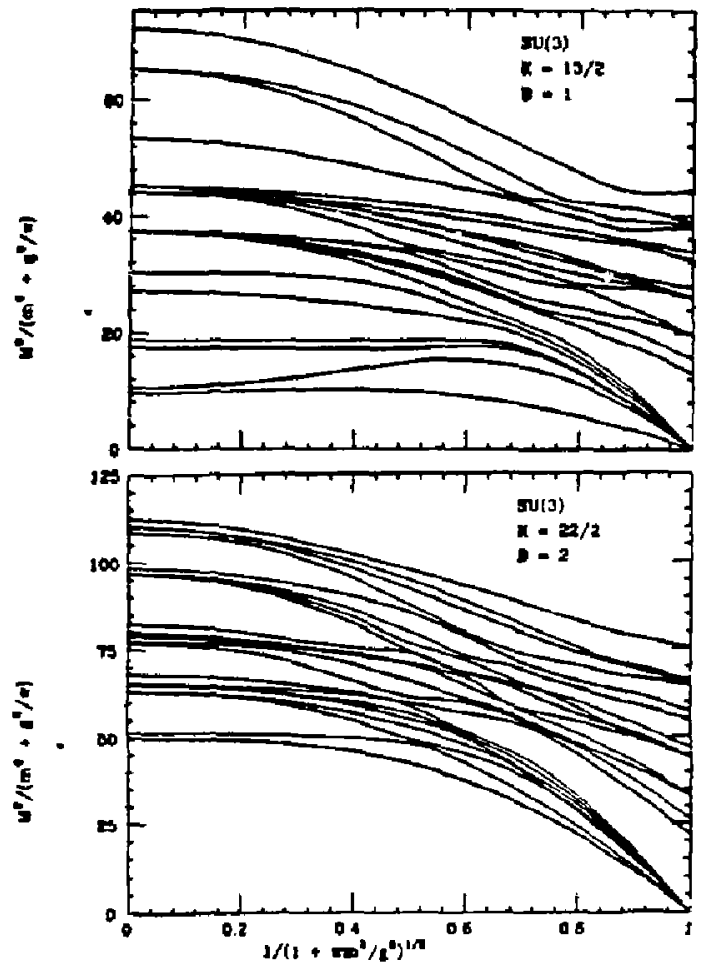

(b)

(c)

Figure 4. Developrnint of $\alpha=3$ Spectrum witl Coupling Constant $\lambda$ (aj Meson Spectivit. $(B-0)$, (b) Baryon Specirum $(B=1)$. (c) Tro Baryon Spersuna ( $B=2$ ) 
which is then diagonalized. The cost in processor time to diagonalize $H$ is insigniffcant compared to that of computing it once; after computing these spectra at some $\lambda$, the rest come essentially for free.

At $\lambda=0$, the spectrum consists of collections of free massive quarks grouped into color singlets, beginning at $M^{2}=m^{2}$ and $9 m^{2}$ for $B=0$ and 1, respectively: Higher states at this coupling have quarks with relative momentum and extra $q \bar{q}$ pairs. For $B=2$, fermi statistics prohibit the quarks from having zero relative momenta; presumably in the continuum linit, as this restriction becomes los: important, the lowest mass will drop to $36 \mathrm{~m}^{2}$.

As the interaction in turned on and increased, the degeneracy of the noninteracting quarks is split, and a fair number of pseudo-crossings become apparent between the weak and strong coupling limits. At infinitely large $g$, a large number of states collapse to zero mass. As will be discussed later, these are composed of a single massless meson, baryon, or baryon pairs ( for $B=0,1$, and 2 ) together with massless states created by adding extra massless mesons to these. In four dimensions, two massless particles can be combined to form a zero mass system when collinear; in two dimensions, all particles are collinear. Also, degeneracie's in the higher states reappear, as might be expected. Combining a massless meson with some state can produce a degenerate state in the absence of interaciouns, as will be shown to be the case.

Spectra with $B=0,1$, and 2 are presented here, but ssstems with arbityarit: large numbers of baryons may be studjed by selecting the appropriate baryus number to see if, among other things. there is interesting nuclear physics in tw... dimensicis. In particular, because baryon number is conserved, the liglutesi $B=$ " 


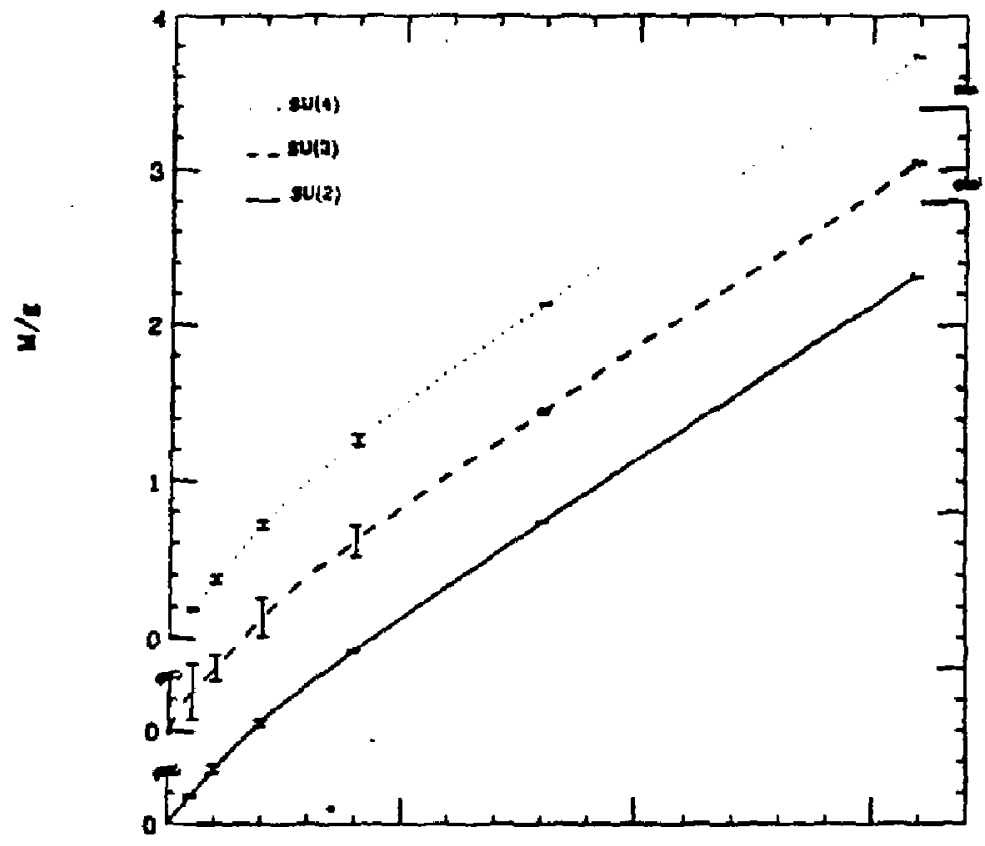

(a)

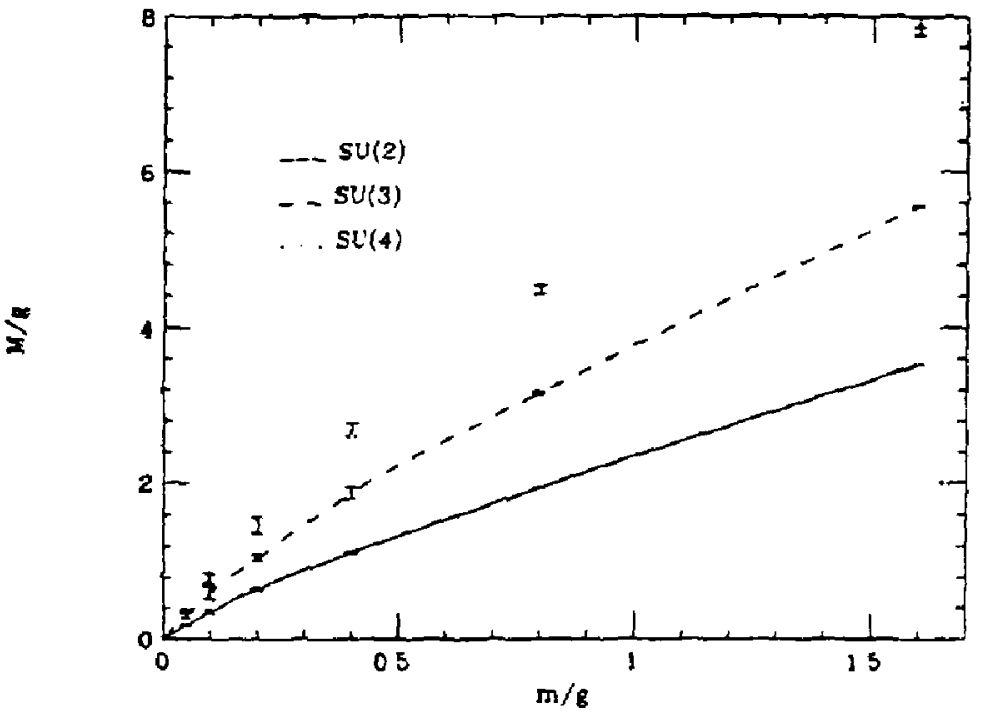

(b)

Figure 5. Lightest Mleson and Baryon Mass versus Quark Mlass for $\Lambda=2,3$ and id la Mesor. (bjBaryoli 
state mass (for example) state mass can be compared to twice that of $B=1$ to see if quark interactions saturate within each baryon, and if not, to compute the nuclear binding energy.

The masses of the lowest-lying states are generally of the most interest and are the quantities most likely available for comparison from other methods, especially: lattice calculations. The masses of the lowest-lying meson and baryon for $N$ of 2,3 , and 4 at a selected set of couplings are listed in Table [3] and displayed in Figs. (5a) and (5b).

Table [3]

\begin{tabular}{|c|c|c|c|c|c|c|}
\hline$\lambda$ & $\mathrm{m} / \mathrm{g}$ & $\begin{array}{c}M_{\text {mes }} / g \\
N=2\end{array}$ & 3 & 4 & $\begin{array}{c}M_{\text {bar }} / g \\
3\end{array}$ & 4 \\
\hline .3325 & 1.6 & $4.314(4)$ & $4.618(6)$ & $4.845(2)$ & $10.71(2)$ & $21.2(3)$ \\
\hline .5763 & .8 & $3.913(4)$ & $4.40(5)$ & $4.743(2)$ & $10.4(1)$ & $20.9(5)$ \\
\hline .8158 & .4 & $2.51(5)$ & $3.1(5)$ & $3.4(2)$ & $7.3(6)$ & $15(1)$ \\
\hline .9425 & .2 & $1.17(7)$ & $1.5(5)$ & $1.4(1)$ & $3.1(2)$ & $6.0(8)$ \\
\hline .9847 & .1 & $.38(5)$ & $.5(2)$ & $.43(5)$ & $1.1(3)$ & $1.9(3)$ \\
\hline .9961 & .05 & $.10(1)$ & $.2(2)$ & $.12(1)$ & $.31(9)$ & $.42(6)$ \\
\hline 1.0 & 0 & 0 & 0 & 0 & 0 & 0 \\
\hline
\end{tabular}

In all cases, that is, at every $\lambda$ and $K$, the lightest $N=2$ meson and baryon have identical masses, and that $M_{\text {mes }} / M_{\text {bat }}=1$ for $\hat{N}=2$ is an exact result. The results quoted in Table $[3]$ are extrapo' 4 s to continuum results by matching to a serjes in $1 / K$ for $2 K$ in the range of roughly 16 to 24 , as will be discussed in Chapter (3). The numbers in parenthesis give the magnitude of the last term in the series fit. For $\lambda \leqslant .9425$, these are reason $z$ ble estimates of the actual error. Bey rod 
this, the largest $K$ employed is likely not large enough for these to be more than a rough guide, as will be discussed. However, when $m / g=0(\lambda=1)$ identically; the lightest state for any $N$ or $B$ is exactly zero, independent of $K$. The nature of solutions in this limit will be discussed below.

Although the lightest $N=2$ meson and baryons have identical mass, the spectra are not equivalent. In particular the masses of the next lightest $N=2$ meson and baryon are listed in Table [4], in units of the lightest mass. This ratio measured at rarious $K$ is more stable than the absolute value, and is likely to give a more accurate extrapolation.

Table [4]

\begin{tabular}{|cc|cc|}
\hline & & \multicolumn{3}{|c|}{ MS $_{(2 \text { ndstate })} / M_{(1) T}$} \\
$\lambda$ & $\mathrm{m} / \mathrm{g}$ & $B=0$ & 1 \\
\hline .3325 & 1.6 & $1.33(1)$ & $1.59(3)$ \\
.5763 & .8 & $1.73(2)$ & $2.41(2)$ \\
.8158 & .4 & $2.4(1)$ & $3.7(2)$ \\
.9425 & .2 & $2.8(1)$ & $3.7(4)$ \\
.9847 & .1 & $2.8(1)$ & $3.7(3)$ \\
.9901 & .05 & $2.6(2)$ & $3.6(3)$ \\
\hline
\end{tabular}

Results for $\lambda$ beyond .9425 should be regarded only as estimates at the values of $K$ used here. In particular, it is not certain that these ratios are actually reaching a fixed value.

It might be worth mentioning that at strong coupling but insufficiently large' $K$, the numerical results for masses may be duplicated by using the exact infiatu" 
coupling wavefunctions to compute the Hamiltonian's expectation value. At infinite coupling (or zero mass) the wavefunctions do not vanish at smail $x$; for non-zero mass, the kinetic terns $m^{2} / x_{1}$ will force them to zero. However, the smallest available pumerical $x$ is $1 / 2 K$. When $m / g$ is small, the kinetic term will not be felt until $K$ is large enough to compensate. A great deal of information in contained in this small-r region, and until this $K$ is reached the continuum 1 nnit is still far off for quantities sensitive to this region.

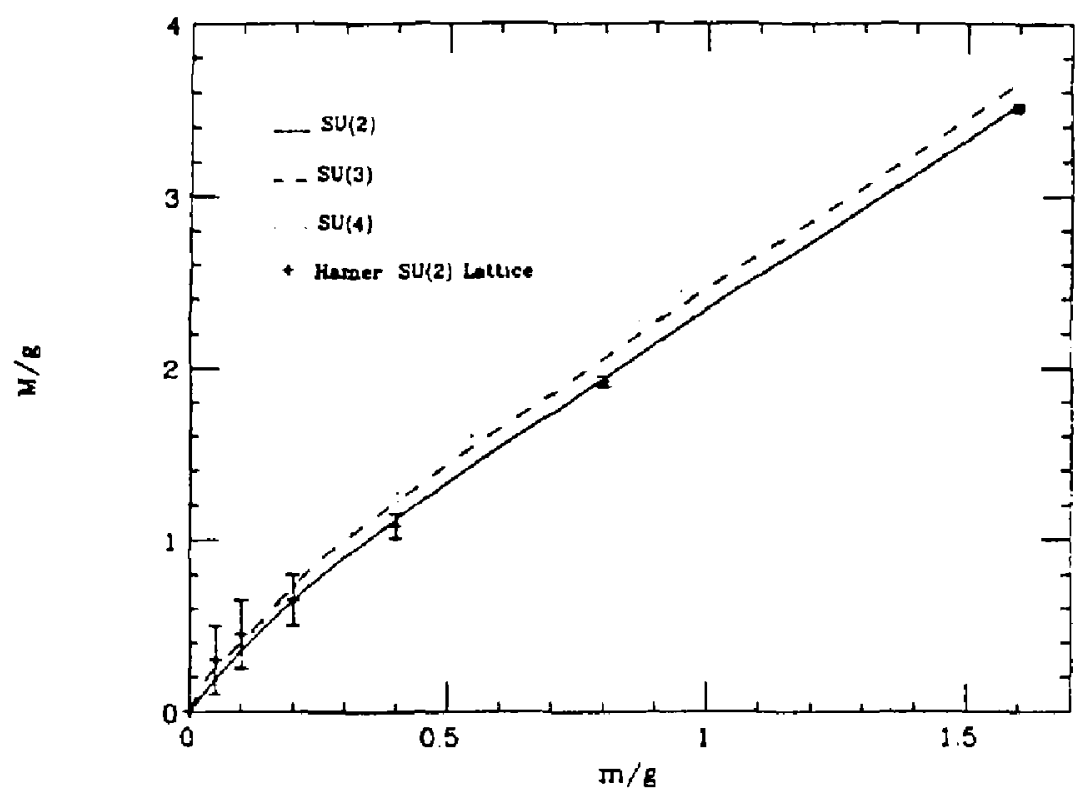

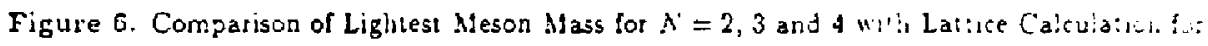
$A=2$.

In ceriain cases, results from this work may be compated with those froni o:..." sources. In particular, Hamer has computed the mass of the lightest $\mathcal{N}=2 \mathrm{n} \cdots \cdots$ and baryon using a Hamilionian lattice with hijson femions. ${ }^{i 26}$ His resulis a:e 
plotted in Fig. (6) along with the results from this calculation for $N$ from 2 to 4, as in Fig. (5a). For ease of comparison, only his error bars are displayed here. Also, the actual data points from this work sit on the same $\mathrm{m} / \mathrm{g}$ locations as his: the curves are fits intended only as guides.

Clearly the agreement is quite good. This is especially reassuring, as the meth. ods employed are very different. In particular, Hamer works in a different gauge $\left(A^{0}=0\right.$ versus $\left.A^{+}=0\right)$, a different space (position rather than momentum), a different quantization prescription (equal-time versus light-cone), uses a differant infrared regulator (lattice size versus isolating by discretization and discarding zero momentum states) and takes a different continuum limit (lattice spacirg versus box size in $\left.x^{-}\right)$. Both methods find accuracy in the chiral limit $(\mathrm{m} / \mathrm{g} \rightarrow 0)$ increasingly difficult, but much less so at $m / g=0$ identically. As is well known. implementing chiral symmetry in a lattice formulation requires some effort, and is related to the difficulty in adequately defining single derivatives for fermions. For light-care quanization, the diffculty is from a seemingly unrelated source. In particular. as $m / g \rightarrow 0$. the kinetic term $m^{2} / x$ which forces the wavefunction to zero in this limit becomes dominant at increasingly smaller values of $x$; as a result, the wavefurction turn-over in $x$ becomes more drastic, and ever larger values of $K$ are necessar: to adequately sample this region. However, as will be shown. this severe behavior is necessary to compensate for the very asymmetric treatment $w !$ ich $2 \cdot L$ and $: \cdot k$ receive duting quantization. Although the constraint equation for 42 ajplea:s $w$ condemn it to decouple as $m \rightarrow 0$, this endpoint behavior for wavefunctions ciat: pensates so that matrix elements with $\tau_{L}$ couple symmerricaliy with those li, $t$, : Because the system is quantized in momertun: spane there is no diffcu! : $\cdots: !$ 
fermion doubling. Perbaps the price to pay is that the fermion mass must be ini. tially finit. co ensure that $\psi_{L}$ doesn't disappear, but that as $m \rightarrow 0$, wavefunctions become increasingly ill-behaved, making numerical accuracy difficult to obtain.

The study of $S U(N)$ in I +1 dimensions began with 't Hooft ${ }_{+}^{[2]}$ who solved the meson spectrum in the large- $N$ limit. By solving for these mesons at finite $\lambda$ here, it is possible to determine both bow good the approximation is at a particular $N$ of interest (three, for instance) as well as how quickly the large- $N$ limit is approached.

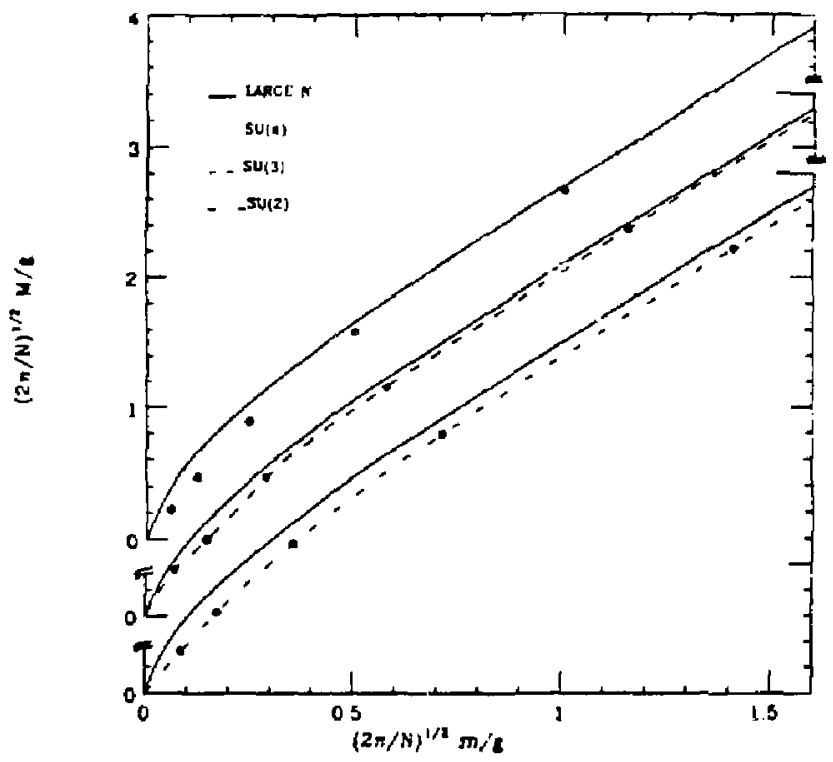

Figure 7 . Comparison of Lighest Meson Mass for $M=2,3$ and 4 with Large- $M$ Mes $\rightarrow$ frout Ref [21]

In Fig. (7), the lightest meson masses for $N=2.3$, and 4 are compared wit 6 i Hooft's lightest large- $N$ meson. Masses in this plot are rescaled into units of $g .1 \%$. as this is the expansion parameter conside:ed fixed as $X \rightarrow \infty$ Even foi $l=?$ 
there is surprisingly good agreement, except for small but finite $\mathrm{m} / \mathrm{g}$, where the light-cone numerical results are not sufficiently accurate for comparison. . ncreasing $N$ from two to four brings results to almost within ertors of the large $N$ limit for most $\mathrm{m} / \mathrm{g}$.

In assessing the large- $N$ approximation, two different questions can be addressed. First, how good a description does the leading large- $N$ term provide of soluticns at a particular finite $\Lambda^{\prime}$; that is, is it accurate? And second. do actual measured quastities scale with $\mathrm{N}$ as assumed in this scheme; that is, is it consisient? Evidently, for the lightest meson, it is accurate at all $N$ and $\mathrm{m} / \mathrm{g}$. The latter is somewhat surprising: because the relevant coupling is really $g^{2} \mathrm{~N} / \mathrm{m}^{2}$, an expansion in this parameter is unreliable at small enough $m$.

Baryon masses are expected to scale proportionally with $\Lambda$, based mainly on nonrelativistic reasoning. ${ }^{[20.301}$ Consequently, baryon masses are infinite in this limit and they decouple. From Fig. (5b), unlike Fig. (7), it is clear that two is no: a large number. In fact. for $N=2$. the lightest baryon has the same mass as the meson, and so it is not reasonable to neglect it. Nevertheless, for most values of $m / g$, the baryon mass does indeed scale with $A$. For any finite fixed $m / g$. the baryor will decouple at large enough $X$

In the linit that $\mathrm{m} / \mathrm{g} \rightarrow 0, \mathrm{f}$ fixed. the bayon mass goes to zero: as $m$ decreases, the $X$ at which this approximation is sensible must inctease: cr. fo: fixed $\lambda$. the approximation becomes progressively less accurate as $m$ decreazis. Also. it wij! be argued that in the vaience quark approvimation, the baryon raa:is proportio:s: to nig. $l^{1 / 2}$ as $n-0$. and so at least in this approximatio. li... 
baryon mass grows as $N^{1 / 2}$ rather than $\Lambda^{\circ}$. The inclusion of higher Foct states will adjust this, but most likely not enough to restore the power to one.

The surprisingly good agreement for mesons even at $N=2$ may be understood by noting that the large- $\Lambda^{\prime}$ meson Bethe-Salpeter equation derived diagrammatically in Ref. [21] is identical to that derived for the light-cone Schrodingr equation restricted to the $q \bar{q}$ Fock space as in Chapter (3), after replacing the coupling $g^{2}\left(\Lambda^{2}-1\right) / 2 \Lambda$ by its large- $N$ limit, $g^{2} \Lambda / 2$. If the valence approximation for mesons is accurate, then, insofar as $\left(N^{2}-1\right) / 2 N$ is close to $N / 2$, the large- $\lambda^{*}$ approximation must be accurate as vell. For large quark mass $m$. this is guarar:teed to be the case; for identically zero $m$, it will also be shown that a massles: meson can be created from only a single $q \bar{q}$ pair. It is then not surprising that the valence approximation, which is exact at both extremes. is reasonable at all $\mathrm{m} / \mathrm{g}$. Because this is true regardless of $\Lambda$, the accuracy of the large- $N$ approximation at these small $\Lambda$ should probably be considered coincidental.

Finally, converting the program to solve for the gauge group ( $(\mathrm{N})$; as ofposed to $S L^{\prime}(N)$ involves only removing the term in the interaction which cancels the trace. This is equivalent to crossing $S(T)$ with massive electrodynamics. or $(1)$. In the large- $N$ limit, $U\left(N^{*}\right)$ and $S L^{\prime}(\Lambda)$ are identical, and so their actual difference at finite $\lambda$ gives another indication of accuracy. The liglitest meson masses fur [(2) and $S L^{\prime}(2)$ are plotted in Fig. (8). In particular. for most values of $m i t$. $L\{2\}$ is even closer to the large. $A$ meson than $S(Y 2)$. because in the $q \bar{g}$ integra: equation, to be presented in Chapter (3), the factor $g^{2}\left(X^{2}-1\right) / 2 \%$ is replaced by its large .7 limit. $g^{2} x / 2$, for $l(2)$. However, there is another term which wi:

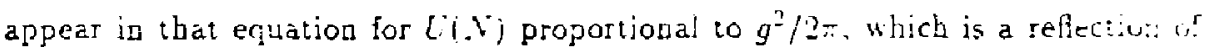




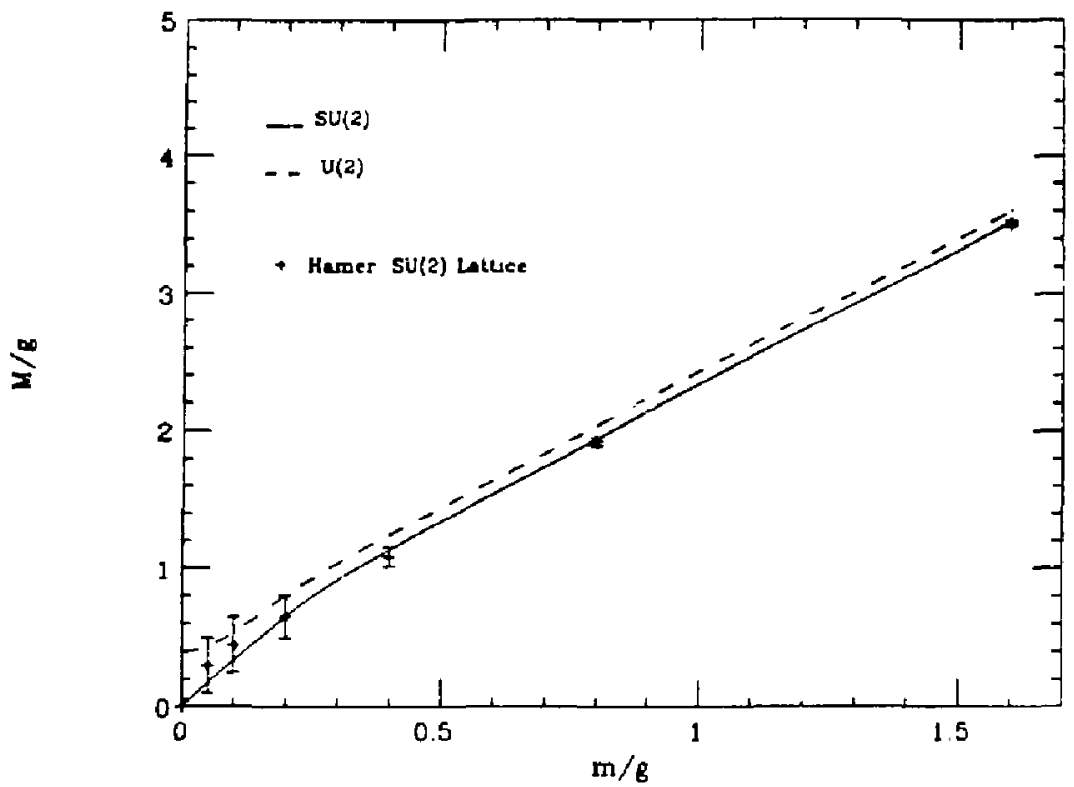

Figure B. Lightest Mleson Mass for $S L^{\prime}(2)$ and $L(2)$

the axial anomaly that gives the Schwinger Model boson its mass. In fact, for all $\left[(x)\right.$, the lightest meson at $m=0$ will have a mass of $g(2-2)^{1 / 2}$. Because $g^{2} . Y$ is considered fxed as $x \rightarrow \infty$, this mass is neglected to leading order ini $1 / x$.

As an aside. the particular case of $L(1)$, or the massive Schwinger node!. was also studied. The program produces results consistent with those of Rer? [20]. These are thenselves consistent witl other calculations of this model and. in particular. give exact results for massless electrons. 


\subsection{QUARK-ANTIQUARK VALENCE WAVEFLNCTIONS}

Because the wavefunction in the valence approximation involves only a $q \bar{q}$ pair. it is possible to plot the wavefunction directly. This is done in Figs. (9a-e) for the lowest-lying $N=3$ meson and the first three excited states as $\mathrm{m} / \mathrm{g}$ ranges from 1.6 to .1 . In these, $2 K=90$, and the corresponding step size in $x$ is $1 / 90$. As a consequence, the accessible values for $x$ nearest the endpoints are $1 / 90$ and $89 / 90$.

For weak coupling, the wavefunctions are reminiscent of the quantum mechanical (momentum-space) wavefunctions for a particle in a linear potential. and as $\mathrm{m} / \mathrm{g}$ becomes large. that is what they become. The kinetic term $\mathrm{m}^{2} / x$ forces the wavefunctions to zero at $x=0$ and 1 , and as $m$ becomes large relative to $g$, as in Fig. (9a), this endpoint suppression is evidest for an increasing range in $x$.

For bighly excited states with a large number of oscillations, the relative in:portance of the endpoints decreases. As discussed in Reís. [21] and [ 30 ' it is not a bad approximation to incorporate the effect of the kinetic term as simply the boundary condition $\phi(0)=\phi(1)=0$. The $n^{\text {th }}$ excited state $o_{n}$ is analogous 10 tha: of a free particle in a box in $x$,

$$
\phi_{n} \sim \sin n \pi x
$$

with a mass

$$
M_{n}^{2} \sim g_{: \pi n}^{2}
$$

When $m / g$ is very large, $n$ must be large before the actual form of the hinetic tern: is unimportant. For $m / g \sim 0$. this picture will be seen to break down a!togr.tien... From Fig. (Gt). it seens that it is most likry to be accuraie for $\mathrm{mi} / \mathrm{g} \sim 1$. 


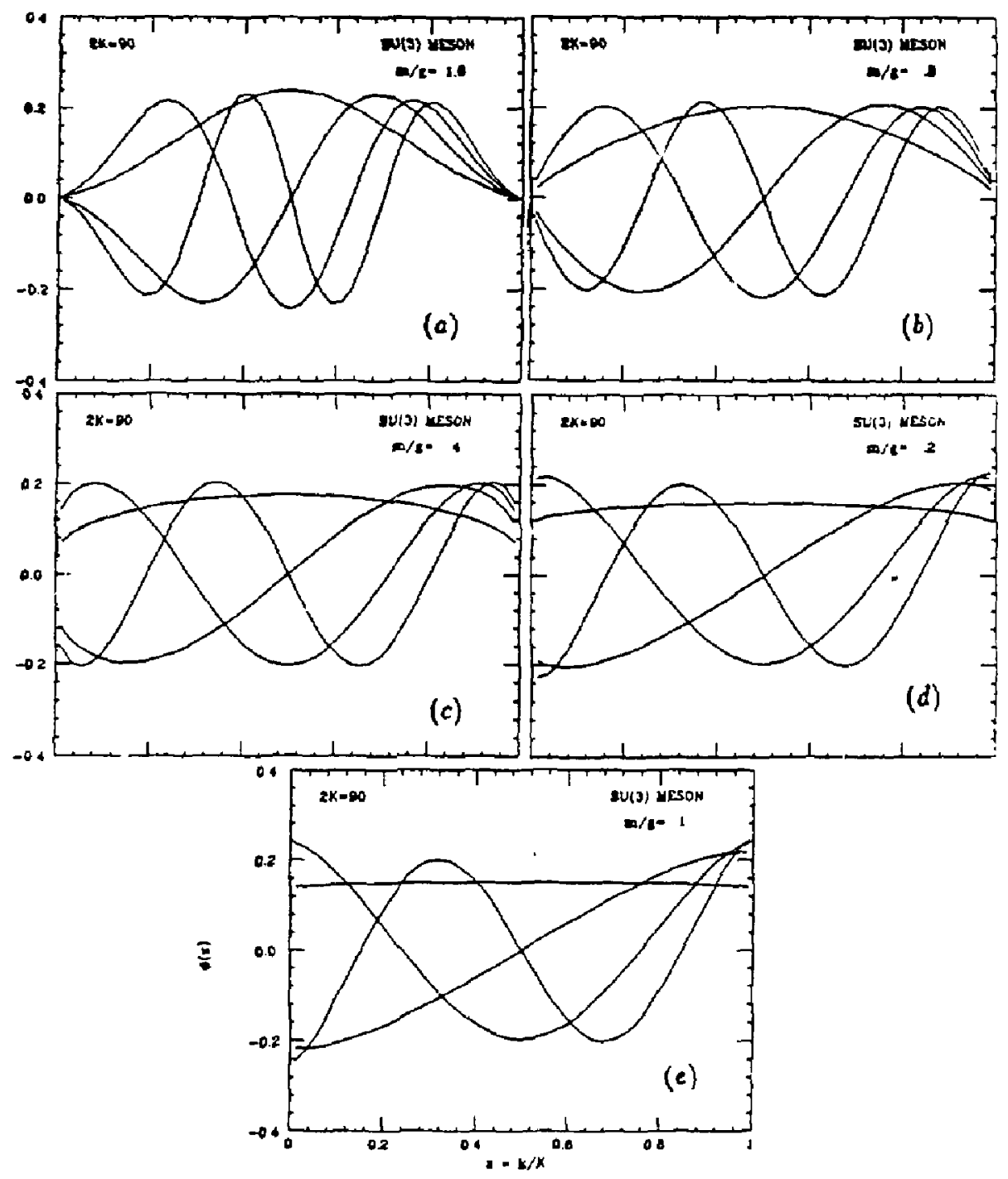

Figuje 9. Wavefugetions for First Five $X=3$ Nesons in $9 \bar{Q}$ (Vaience) Afproximatsr.

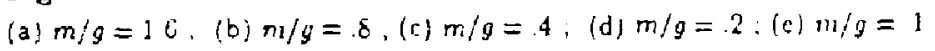


Finally, in the strong coupling limit, as in Fig. (9e) where $m / g=.1$, the interaction term dominates so much so that the turnover of the wavefunction to zero at the ends is not discerriable at this resolution in $x$, that is $\Delta x=1 / 90$. In fact, over most of $x$, the wavefunction is much more like an oscillating string with free rather than fixed ends.

\subsection{STRUCTURE FUNCTIONS}

Apart from valeace mesons, which involve only two particles. it is not couvenient to display wavefunctions directly, due to the large number of potential plotting varjables and the inability to distinguish quarks with the same quantum numbers. The quark (and antiquark) structure functions are defined by

$$
q_{k}=\left\langle\dot{\phi}\left(K^{*}\right)\left|b_{k}^{\dagger c} b_{k, c}\right| \phi\left(K^{\circ}\right)\right\rangle
$$

with $d_{k, c}^{\dagger} d_{k}^{c}$ replacing $b_{k}^{\dagger c} b_{k, c}$ for $\bar{q}_{k}$. They describe the manner in which the momentum $K$ is distributed among the quarks, and have the advantag of being functions of a single variable. The $q_{k}$ automatically satisfy a baryon number sum rule

$$
\sum_{k}\left(q_{k}-\bar{q}_{k}\right)=\left\{\begin{array}{ll}
0 & \text { for mesons } \\
\Lambda & \text { for baryons }
\end{array}\right. \text {, }
$$

the momenturn sum rule

$$
\sum_{k}\left(q_{k}+\bar{q}_{k}\right)=K
$$

and so on. In the continum limit. $k / K$ becomes the Bjorben variable $x$, and $i, \cdot$. 
continuum structure function is $q(x)=K q_{k}$. These are then normalized such that

$$
\int_{0}^{1} d x(q(x)-\bar{q}(x))=0 \text { or } N
$$

for mesons or baryons.

Besides being simple to plot, these functions are physically useful, and are measurable in deep inelastic scaltering, at least in four dimensions. (Note that these are related to the commonly used parton model structure functions $f(x)$ by $x q(x)=f(x)$.) This provides one of the main motivations for attempting to solve $Q C D$ on the light cone. Apart from the apparent numerical advantages, the wavefunctions which result are directly related to experimentally measured quantities.

\subsection{VALEXCE STRUCTLRE FLNCTIONS}

The structure functions $q(r)$ for the lowest-lying $S L^{-}(3)$ meson and baryon are displayed in Fig. (10), at relatively weak $(\mathrm{m} / g=1.6)$ and strong $(\mathrm{m} / g=.1)$ coupling. These correspond to values of .3325 and .9847 for the dimensionless coupling $\lambda$. Specifically, these are the contributions to $g(x)$ from the valence wavefunctions. Hig!her-Fock wavefunctions contribute very little to these states, as wil: be discussed later.

When $m / g$ is large. $k^{+}=\left(k^{2}+m^{2}\right)^{1 / 2}+k$ is dominated by the quark mass. Tin total $P^{+}$is then approximately $m$ times the number of quarks. Consequently. $y(s)$ peaks strongly about $1 / 2$ and $1 / 3$ (or $1 / 3$ ) for the meson and baryon, respectively: As $\mathrm{r} / \mathrm{g}$ decreases. the effect of the quark mass diminishes. and the distritution 


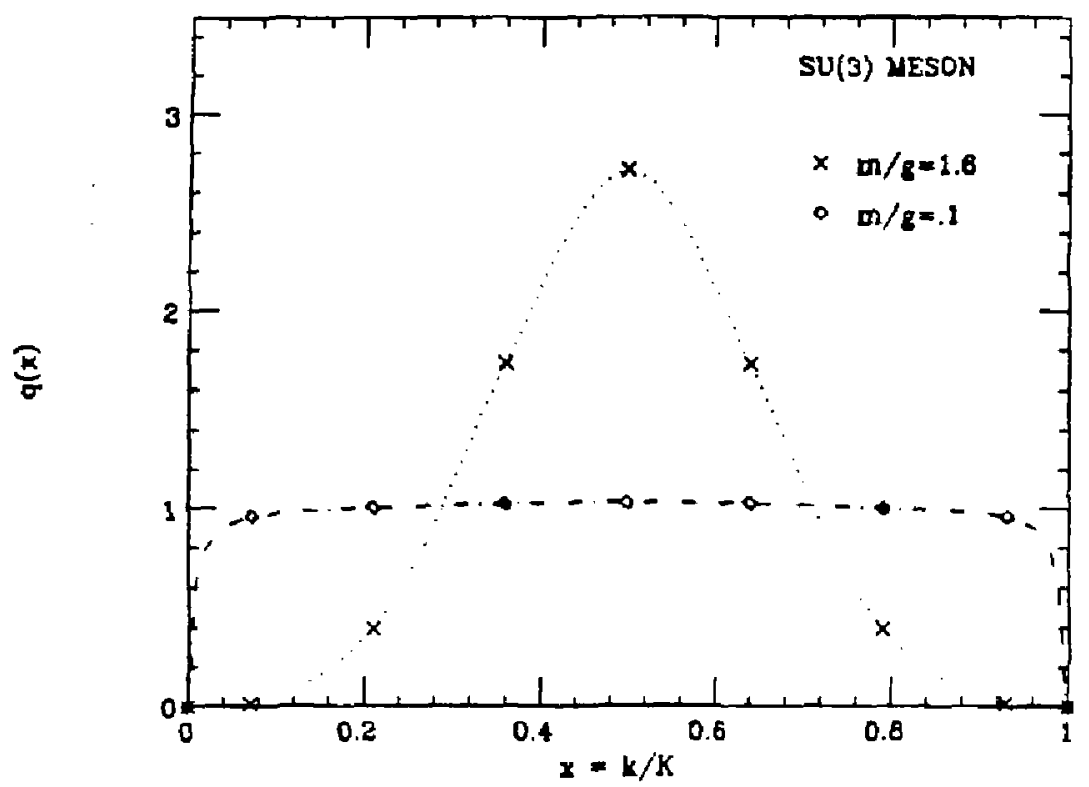

(a)

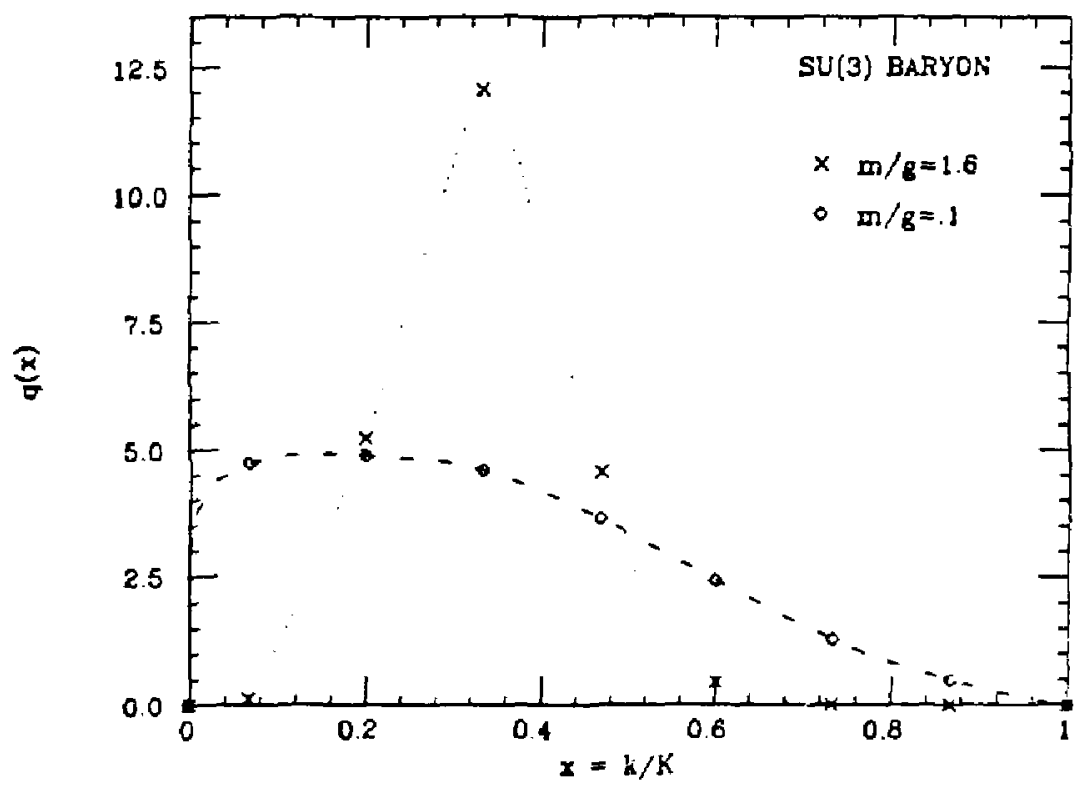

(b)

Figure 10. Siructure Functions for the Liglitest $N=3$ Meson and Baryon (a) Mlesoit it Baryon 
in $x$ spreads. As will be shown, at strong coupling $q(x)$ is governed entirely by: combinatorics; any momentum is as likely as another, subject only to momentum: conservation, $\sum x_{1}=1$. In all cases, wavefunctions, and therefore structure functions, are forced to zero when any quark carries zero momentum due to the kinetic term $\mathrm{m}^{2} / x$. The relative strength of $\mathrm{g} / \mathrm{m}$, which multiplies the interaction tern. determines the $I$ at which this suppression sets in. It should be noted that the value $f f(x)$ at the data points $x=0$ and $l$ in these plots are inferred; the smallest $x$ actually carried by a quark is $1 / 2 \pi$.

\subsection{GENERAL WAVEFUNCTION RESLLTS}

Because of the simplicity of the vacuum, all of the quanta appearing in eigenstates, or wavefunctions, are directly associated with hadrons. Consequently, the nature of a particular state is often relatively simple to deduce from the form of the wavefunction.

In Figs. (11a-c), the valence and four quark contributions to the quark structure function for the first three $\Lambda=3$ meson $(B=0)$ states are plotted for relative'? weak coupling and $2 \pi$ of 24 . These can be interpreted as the $q \bar{\eta}$ mesoln and its first two radial (in $x^{-}$) excitations. Their interpretation is particularly simple because the higher-Fock contribution is so strongly suppressed. due. at least in part. to the large quark mass. Fig. (11d), the eleventh state in the spectrum, is composid predominantly of two $q \bar{q}$ pairs, peaked at $x=1 / 4$. This evidently corresponds tw a pajr of the lightest mesons, and its mass is twice that of the lightest meson.

Corresponding plots for the first three $S C(3)$ baryon $(B=1)$ states are stop:. in Figs. (12a-c). These also represent a predomiranty valence (three-cuait: 

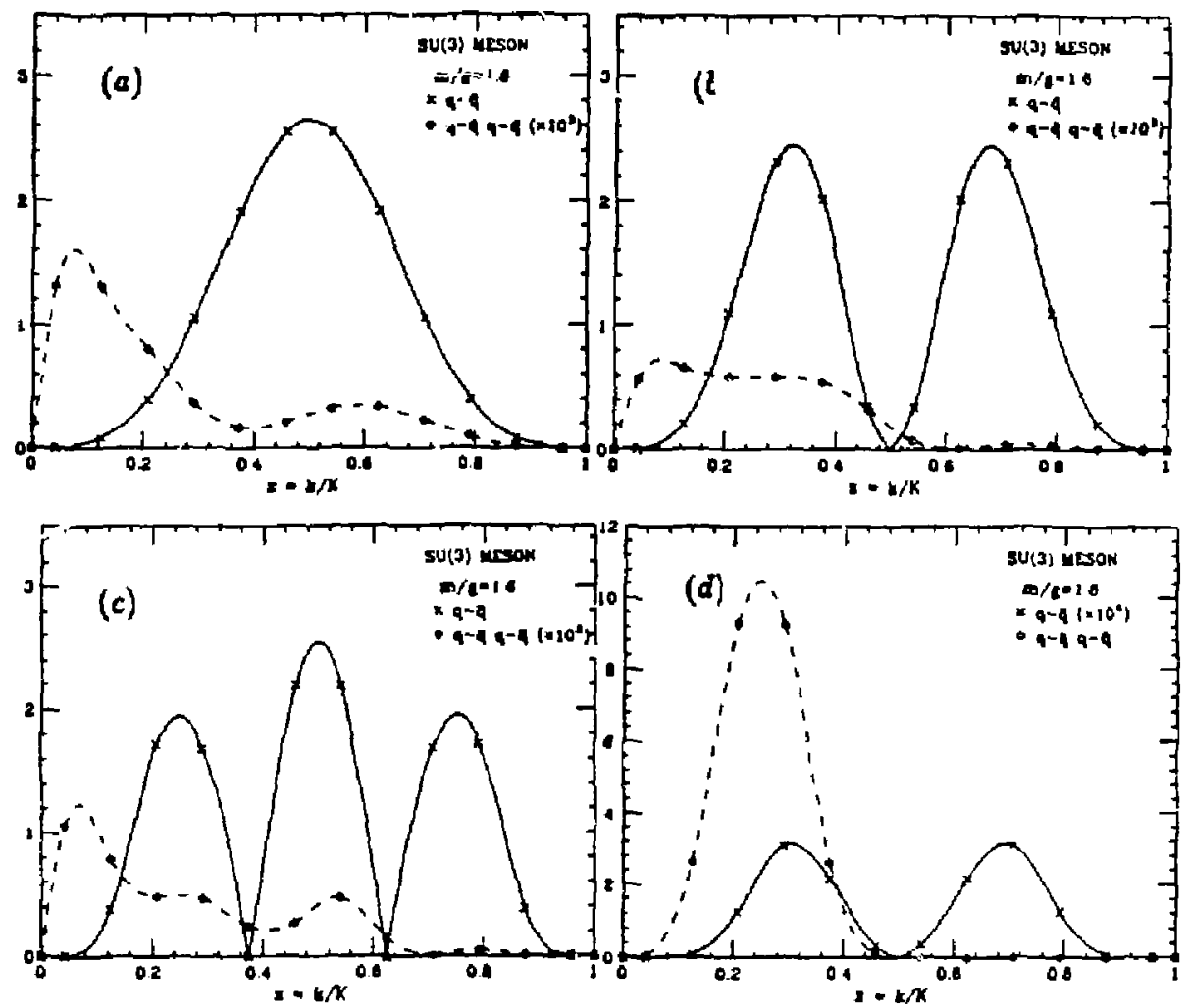

Figure 11. Structure Functions for the Valence and Four-Quath $M=3$ Meson Wiavefunctiats at Weak Cocpling. (a) Lowest State; (b) Second State; (c) Thurd State. (d) Eleventh State 

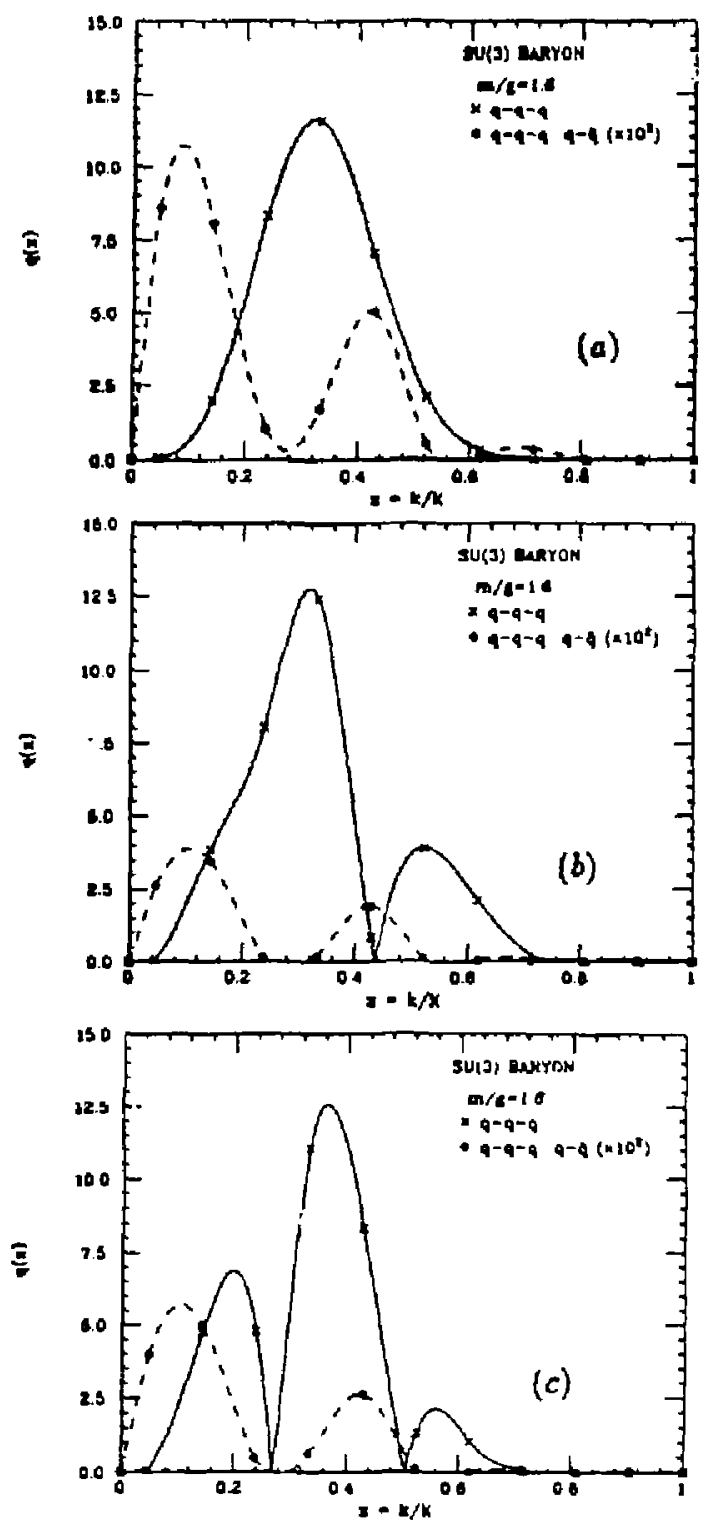

Figure 12. Structure Functions for the Valence and Five-Quark $N=3$ Beryon Wavefuncticis at Weak Coupling. (a) Lowest State. (b) Second State; (c) Third State 

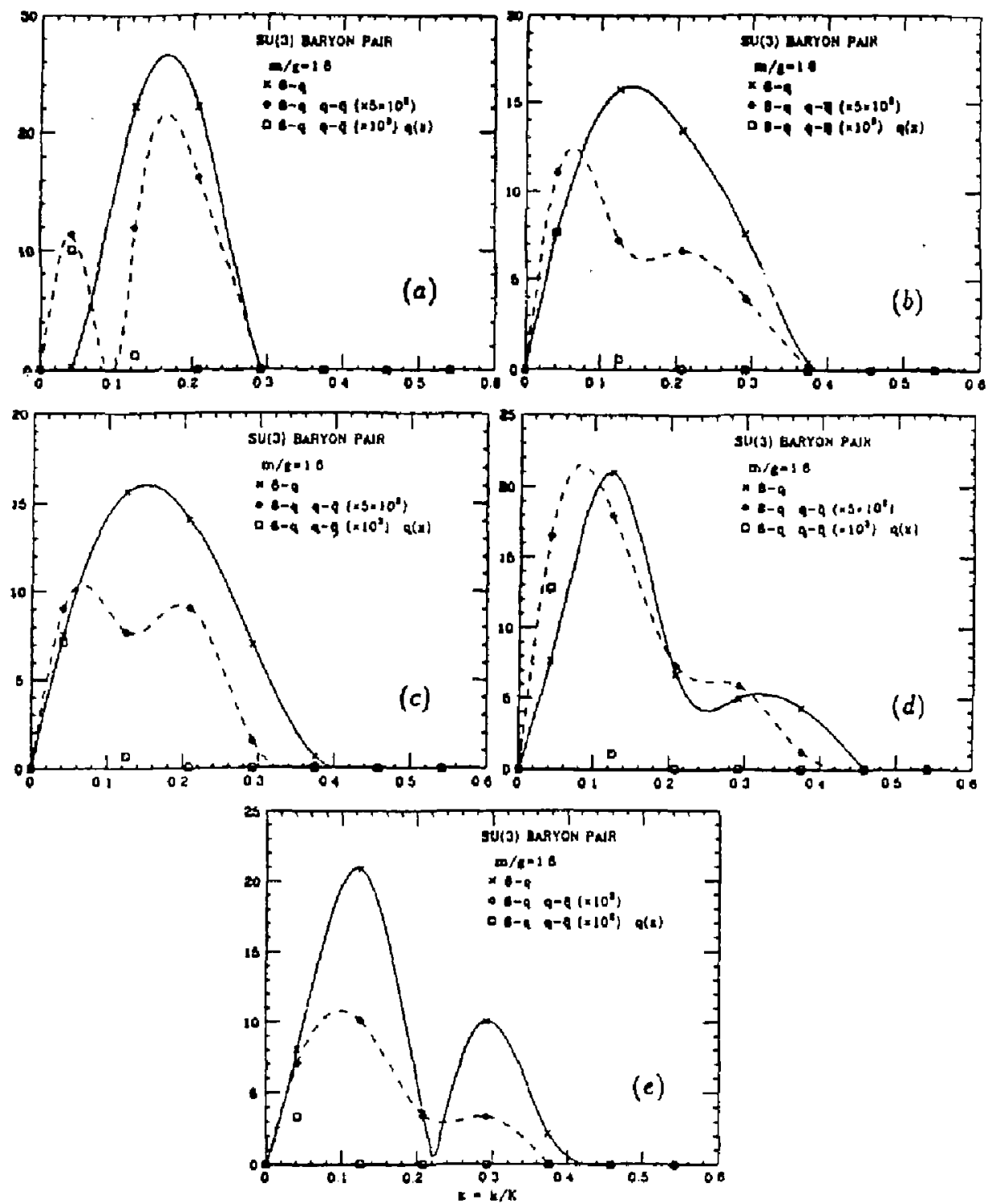

Figure 13. Strueture Functions for the Valence and Eight-Quark $\Lambda=3$ Tro-Baryon Wastfunctions at Weak Coupling (a) Lowest State: (b) Second State, (c) Third State, (d) Fouriti State; fej Fith State. Note the change in Seale 
baryon and its radial excitations, with suppressed contributions from the com. ponent with an extra $q \bar{q}$. The state with a pair of the lightest baryons may be formed as the lightest state in the $B=2$ spectrum, Fig. (13a). Iocluded in this plot is the antiquark function $\bar{g}(x)$, which gives an indjcation of the magnitude and momentum distribution for the meson content of the $S L^{\prime}(3)$ baryon. For compleit' ness, Figs. (13d-e) give the next four $B=2$ states. These are easy to interpret as various combinations of the first few $B=1$ states.

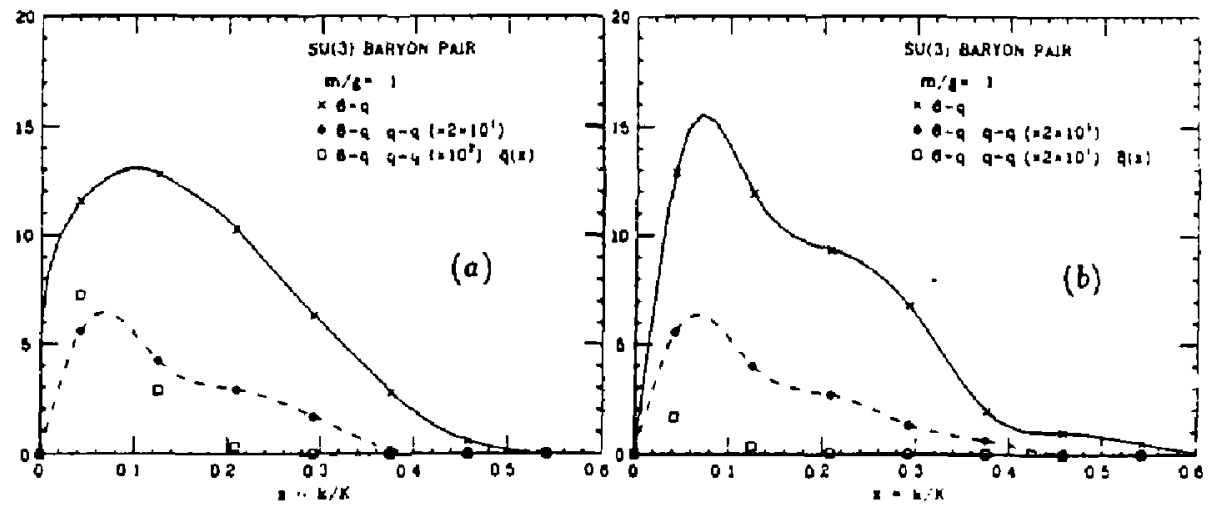

Figure 16. Structure Functions fo: the Valence and Eaght Quath $A=3$ Two-Baryor Water ant tions at Sirong Coupling (a) Lowest State; (b) Second State

In Figs. (14) to (10), the corresponding first several states' structure functivil: are plotted at strong coupling. $m / g=.1$. The connecting surves are simply cul, spline fits: the resolution is in some cases nol good enough for these to accuate depict the actual structure. It is apparent from these plots that as interpreistur.

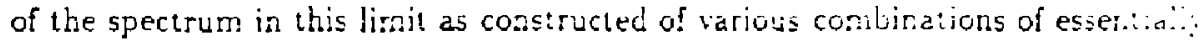

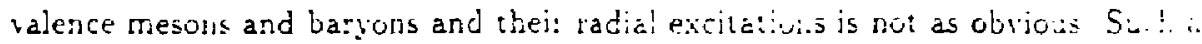




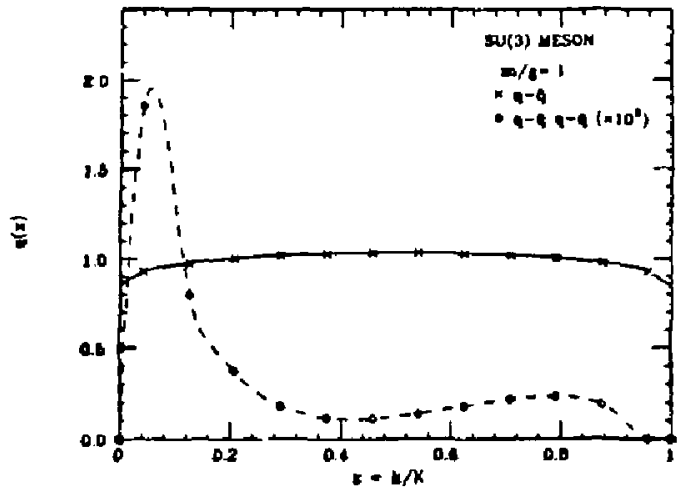

(a)

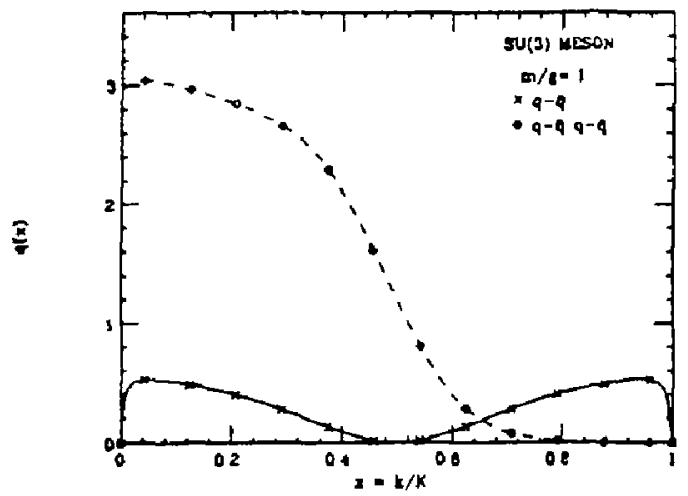

(b)

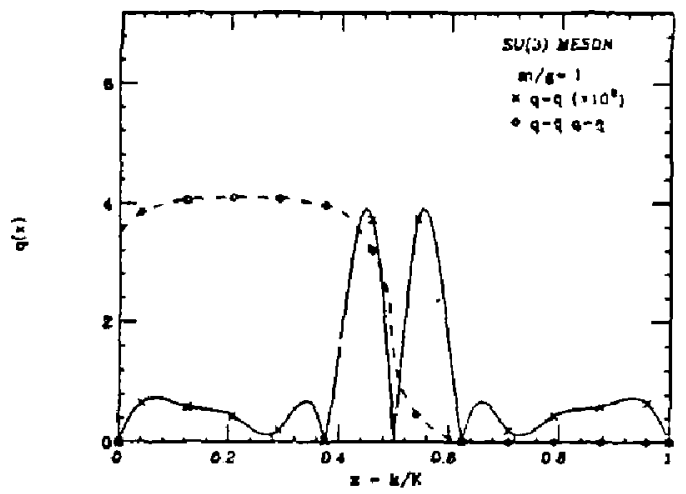

(c)

Figure 14. Structure Functions for the Valence and Four-Qua:k $N=3$ Meson Wavefurctick at Strong Coupling (a) Lowest State; (b) Second State, (c) Third State. 


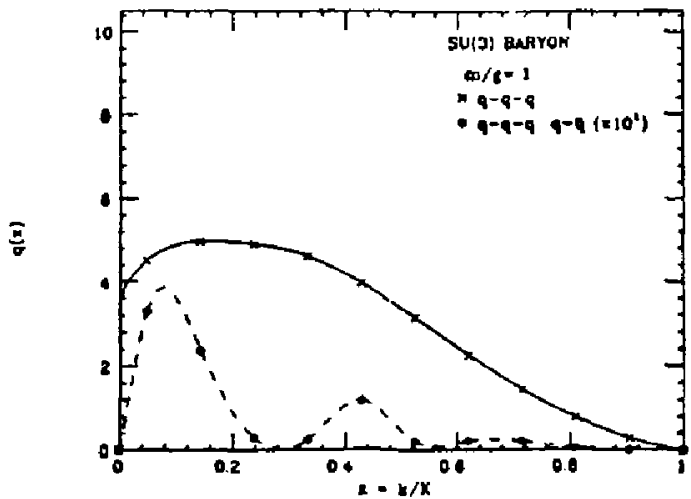

(a)

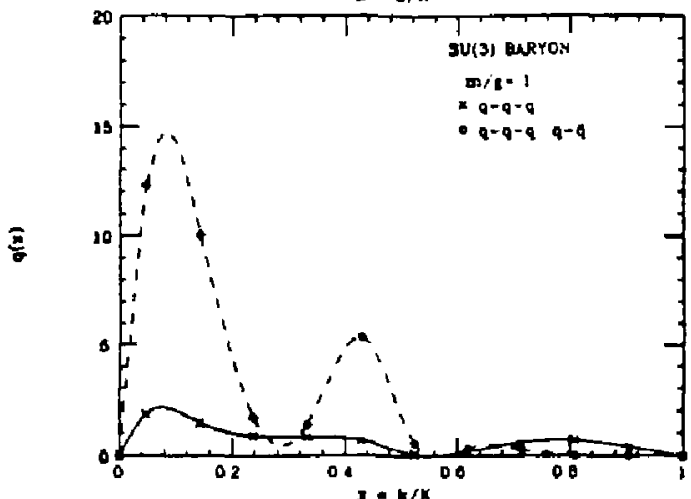

(b)

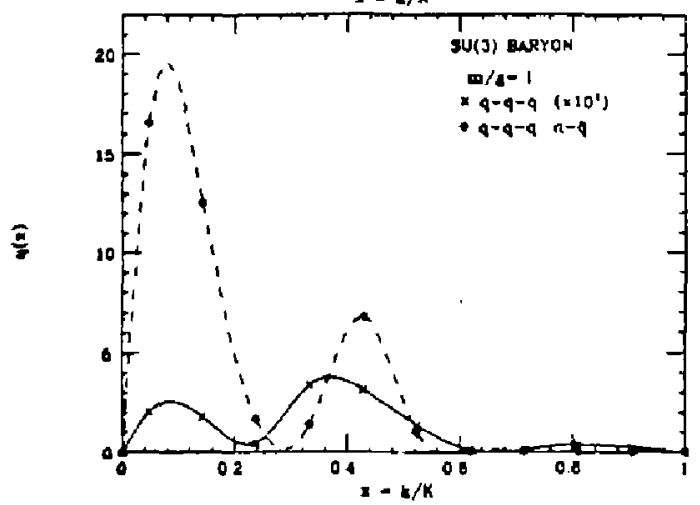

(c)

Figure 15. Structure Functions for the Valence and Five Quark $N=3$ Baryon Wavefunctuon: at Strong Coupling. (a) Lowest State. (b) Second State, (c) Third State 
characterization based on essentic'lly valence hadrons is not even necessarily meaningful. As will be shown, in the continuum limit for vanishing quark mass, it wiil be possible to append extra massless, zero momentum $q \bar{q}$ pairs to existing states witbout altering their mass, producing large degeneracies whicb include stat es with arbitrarily large numbers of quarks.

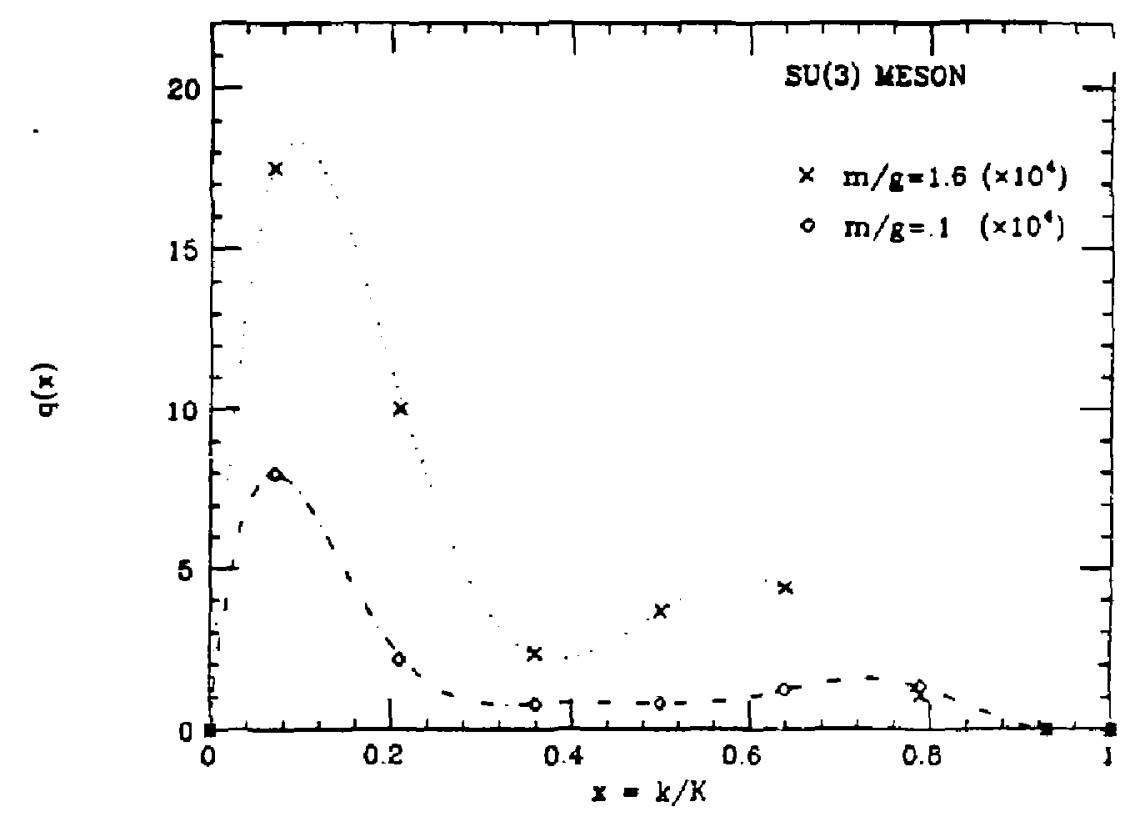

Figure 17. Contribution to Lightest $N=3$ Meson Structure Funttion from Four-Quarh Mavefunction.

One of the advantages of this numerical approach is that it is no: necessai: to make a priori simplifying assumptions regarding the Foc': state strutrure of eigenstates. In particular, the role which higher-Foch states play in the composit iu: of hadronje light-cone wavefunctions can be studied for arbitrarily fine resolutio: i: momentum, subject of course to available computing powes. This is a patent." 
rich field for future work, even in two dimensions, and particularly when favor is included. I $I_{1}$ this section, only the general features of these states will be discussed.

First, for all coupliags $g / m$, the content of the next higher Fock states is strongly suppressed, typically by several orders of magnitude, for the lowest-lying mesons and baryons. The $S U^{\prime}(3)$ badrons in Figs. (17) and (18) are typical. Note that in Fig. (18b), the probability for the state with two extra $q \bar{q}$ pairs is comparably suppressed relative to one extra, and in most cases states with greater than o- pair could be safely neglected. For very massive quarks this is expec ed for all low-lying states, as more quarks mean more mass. It is not clear why it is true when the quark mass is small, although this is only true for the veij lightest staies in this case. Other low-lying states have substantial, even doninant, higher-Fock components.

Second. typical behavjor for the lightest neeson Fock state with one extra quark pair as a function of $R$ is displayed in Fig. (19) for $S C(N)$ and Fig. (20). for $[(1)$. For fixed. week coupling. the content is strongly suppressed but relatively insensitive to $\Lambda$, while at strong coupling it dis inishes rapidy as $\Lambda$ increases. For $(\mathrm{N})$ in this limit, the higher Fock content vanishes aitoget ier is the strü. coupling limit: the massive Schwinger mesan is 'nowo to be composed entirety of a $q \bar{q}$ pair ${ }^{(2.25)}$ Since in this limit these $q \bar{q}$ pairs carry a mass-squared of $g^{2} / 2 \pi$, ex.. pairs cannot mix with the lightest meson wiblout sigtificanty increasing it: naw 


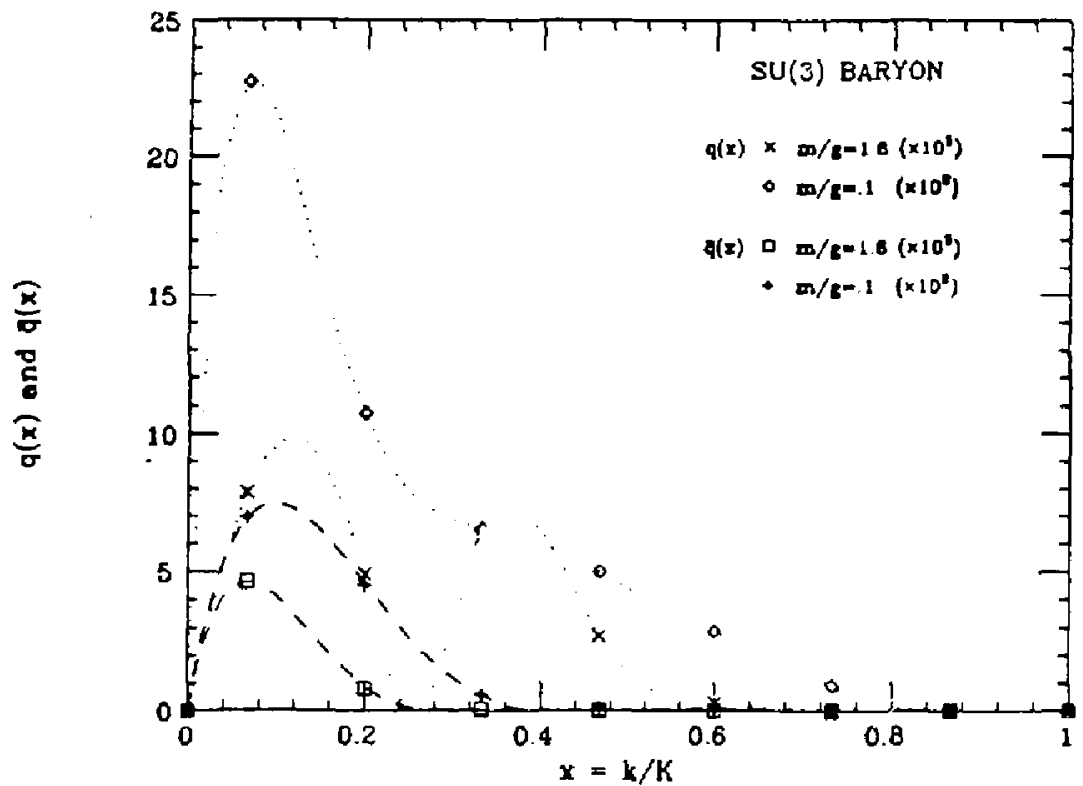

(a)

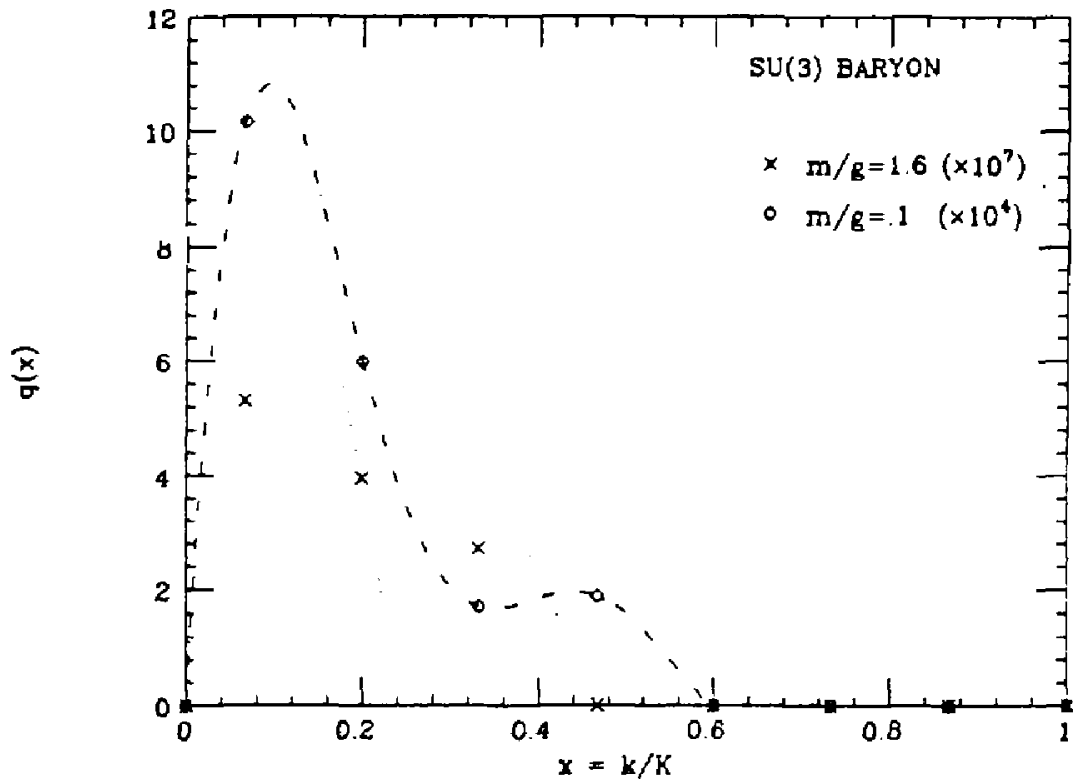

(b)

Figure 18. Contritution to Lightest $N=3$ Baryon Structure Firction. (a) From Five-Qtati Wavefunctuon, (b) From Seven-Quark Wavefunction 

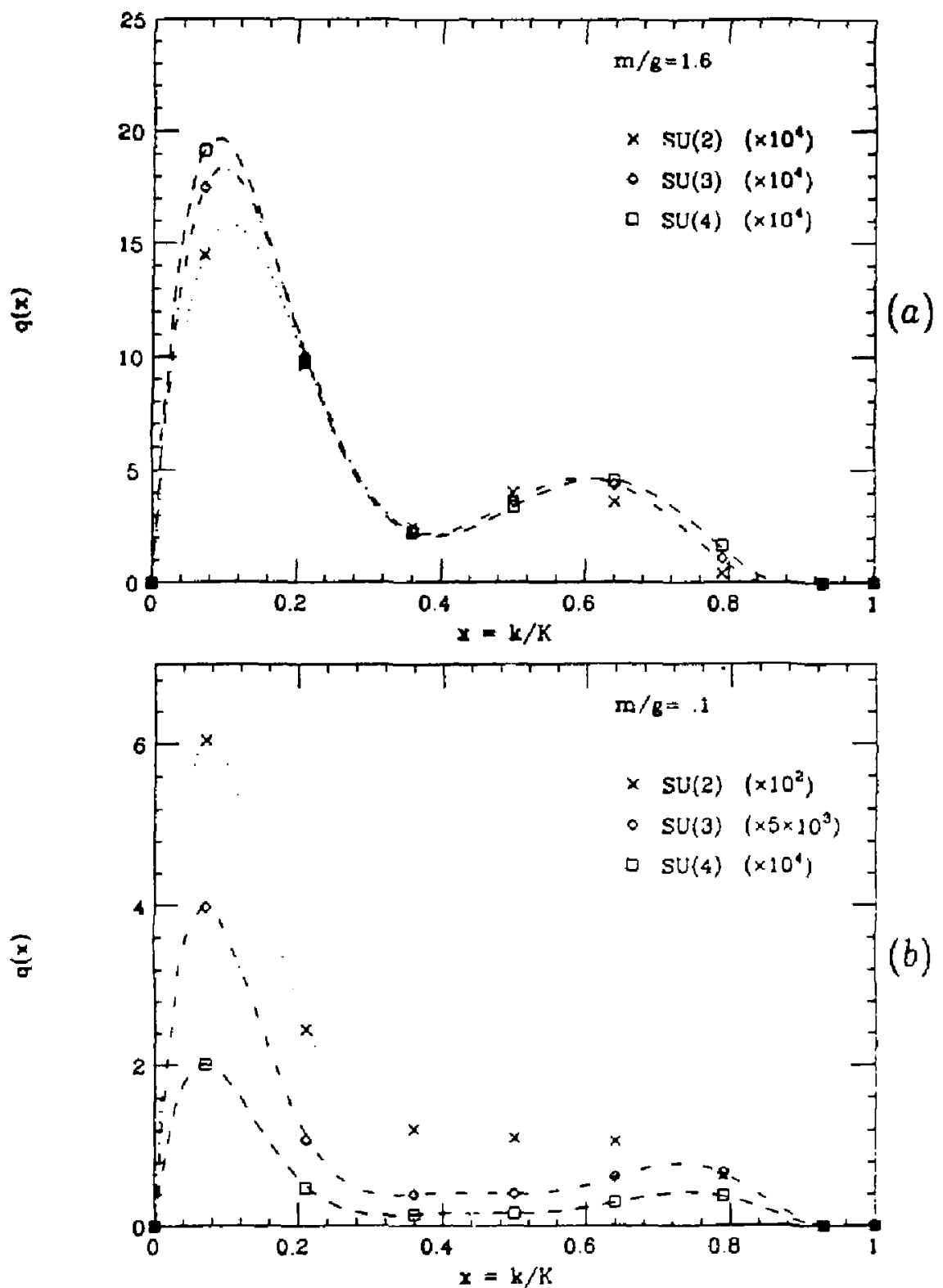

(b)

Figure 19. Contribution to Lightest Meson Structure Function from the Four-Quart Wave: tion as a Function of $\boldsymbol{N}$. (a) Weak Coupling: (b) Strong Coupling 

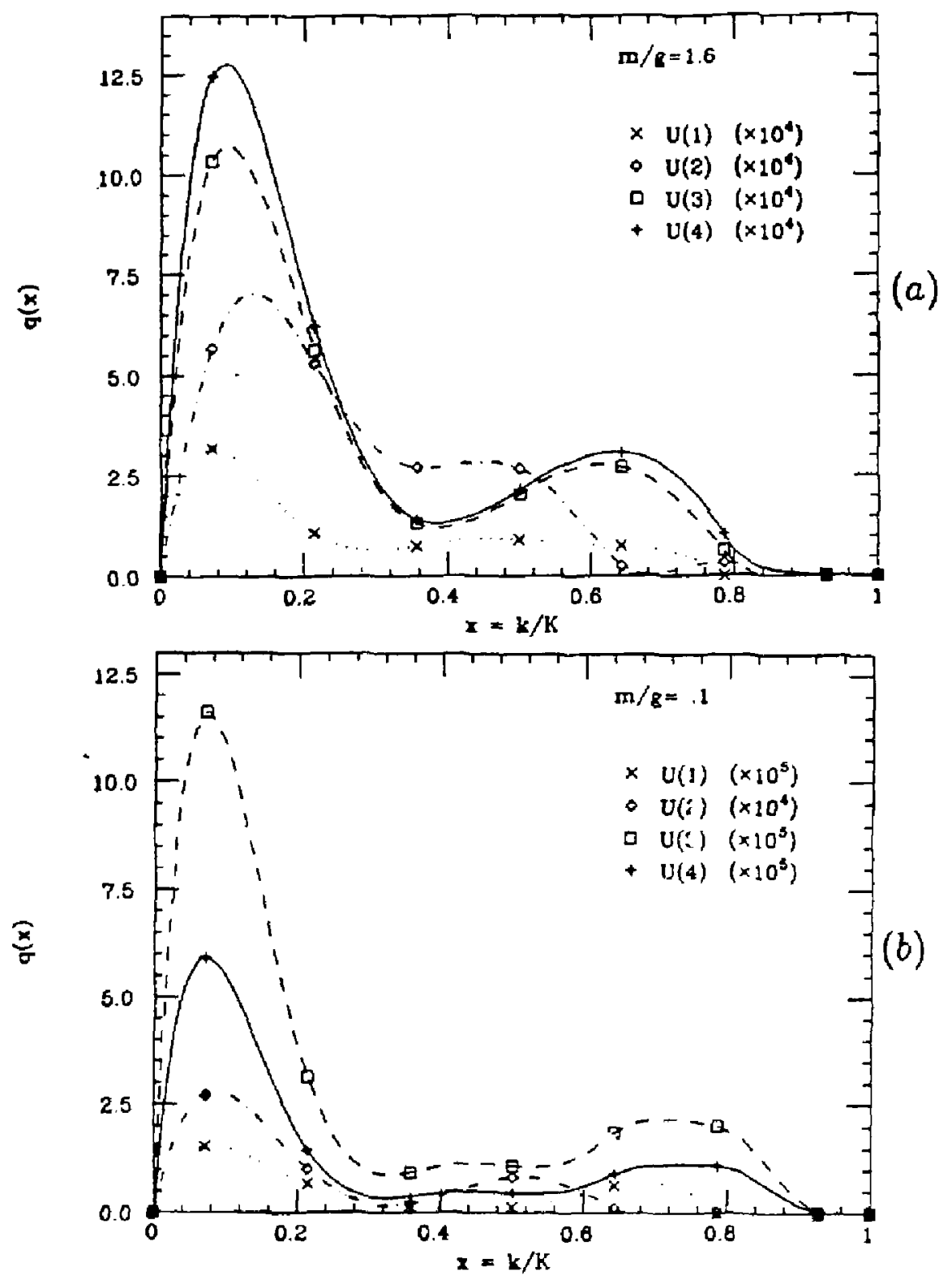

Figure 20. Contritution to Lightest $U\left(N^{\circ}\right)$ Meson Stsu-ture Function from the Four-Quart. Wavefunetion as Function of $N$. (a) Meak Coupling: ( $t$ ) $S$ treng Coupling. 
Table [5]

\begin{tabular}{|lll|}
\hline$N$ & $m / g$ & $1-P(q \bar{q})$ \\
\hline 2 & 1.600 & $.2072 \times 10^{-3}$ \\
3 & 1.960 & $.1325 \times 10^{-3}$ \\
4 & 2.263 & $.9579 \times 10^{-4}$ \\
\hline 2 & .1000 & $.9889 \times 10^{-2}$ \\
3 & .1225 & $.2921 \times 10^{-3}$ \\
4 & .1414 & $.1277 \times 10^{-3}$ \\
\hline
\end{tabular}

In the large- $N$ limit, the higher-Fock content would be expected to vanish, as the meson is composed entirely of valence quarks, and meson-meson couplings are suppressed by an extra power of $N$. In this expansion, $g^{2} N$, rather than $g$, is held fixed. In ordes to determine the importance of bigher-Foch states as a function of $\mathrm{X}$ with this limit in mind, $g$ should be scaled by $N^{-1 / 2}$. In Table [5]: the probability for finding the lowest-lying meson in other than its valence wavefu:rtion is lis:te for $N^{*}=2,3$ and $f$ for both weak and strong coupling. For these resuits. $2 K$ is fixed at 20. Only states with at most two $q \bar{q}$ pairs are incleded. as higher-Fuck states are negligible relative to these. For the weak anc strong coupling cases, $m / g$ is set to 1.6 and 1 . respectivel: for $.1=2$, and $g$ is scaled to keep $g^{2} .1$ fixed for $\Lambda=3$ and 4 .

Clearly the importance of the higher-Fock states dirrinist wat . H. Holdog $g^{2} N$ fixed for increasing $N$ is equivalent to decreasing $g$ or to increasing $m$, so thiresult is not surprising. Larger $N$ means heavier quarks. and it is kinemairát: more costy to baye states with more of them. However th.s does not explai.. …. 


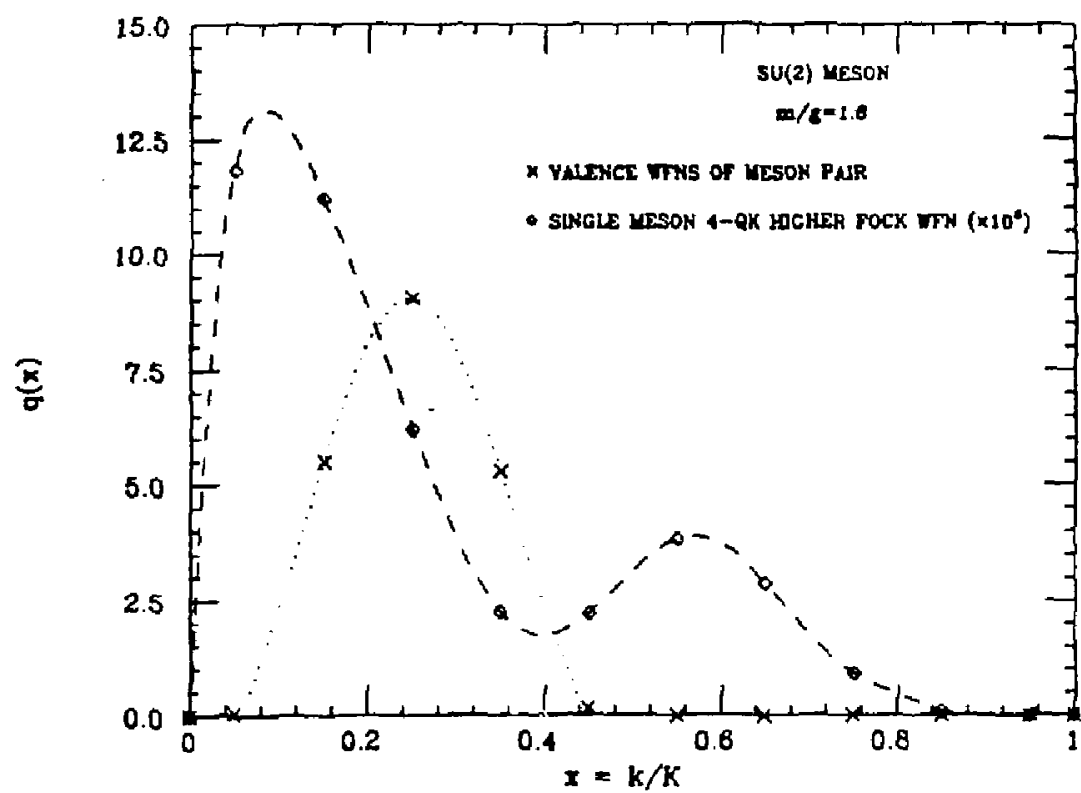

(a)

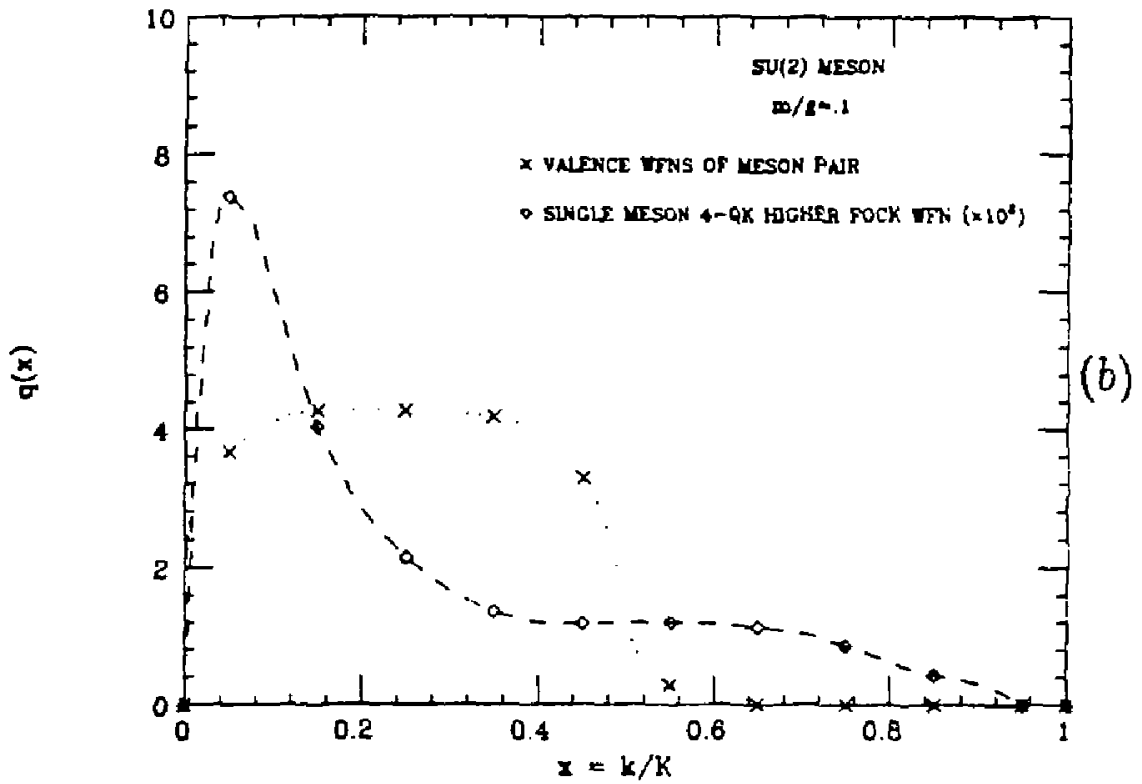

Figure 21. Comparison of Single Meson Four-Quark Bigher-Fock Wavelunetion witb Wavefunition of a Paiz of Valence Mesons (a) Weak Cuupling; (b) Strong Coupling 
even for the very small $\mathrm{m} / \mathrm{g}$ near .1 , this probability is so small to begin with.

Finally, most higher-Fock states share a characteristic, double-bump structure in $I$, regardless of $g / m$ or $N$. It is likely that there are more peaks in these states, but at these values of $K$ the resolution is not high enough to discern any finer structure. Because of this characteristic shape, it is not difficult to distinguish by shape as well as magnitude, for example, four-quark continuum states made from a pair of lighter $q \bar{q}$ mesons from the bigher-Fock component for a single meson, as in Fig. (21).

\subsection{HIGHER-FOCK WAVEFUNCTIONS}

Many successful approximation schemes such as the $1 / N$ expansion for mesons, and phenomenological models, such as the nonrelativistic quark model, begin by describing badrons as predomirantly composed of valence quarks. This is certainly: a reasonable assumption for very massive quarks, but there is no good reason to believe the same for light quarks. In fact, the probability of finding a badron composed of light quarks in its valence state could conceivably be negligible. Because no cuch approximation need be made in the approach hese, it is possible to determine explicitly the importance of higher-Fock states in describing mesons and baryons. at least in tro dimensions. As the quark mass or co:plitig corstant are varied, the region where such a picture breaks down may also be determined.

A rough estimate of tie higher-Fock component to expect in an eigenstate of mass $M$ may be made by comparing the invariant mess of these higher state $t w$ 11. Each Fock state of $n$ particles may be assigneci a mass-squared based on the 
light-cone energies of free quarks,

$$
\Lambda_{n}^{2} \equiv \sum_{i=1}^{n} \frac{m^{2}}{x_{i}}
$$

which is minimum whea all the $x_{1}$ are approximately $1 / n$; that is, when the ligh:cone momentum is equally distributed. In that case,

$$
\Lambda_{n}^{2} Z n: \frac{m^{2}}{1 / n}=n^{2} m^{2}
$$

A Fock state for which $\Lambda_{n}^{2} \gg A^{2}$ should not contribute significantly to a state of that mass. States with $n$ quanta should appear prominertly in such a state only: when their minimum invariant masses satisfy

$$
A_{n \min }^{2} \sim M^{2}
$$

so that, by Eq. (2.8),

$$
n \sim M / m
$$

This is clearly true when the quark mass $m$ is large. In the opposite limit. as the quark mass vanishes,

$$
M \sim(g m)^{\frac{2}{2}}
$$

for all hadrons. and therefore

$$
n \sim\left(\frac{g}{m}\right)^{\frac{2}{2}}
$$

The exponent $1 / 2$ is correct in the large- $X$ limit, and it is likely that it provinde a reasonable estimate ai finte .7 , as will be discussed below. In th: limit, fuc't 
states with any number of quanta can appear. How many actually do nay be determined oumerically:

Consider first the lightest meson in $S U(2)$. The contribution to the wavefunc. tion from higher-Fock states may be measured by how much the probability of finding the mesor in its valence state differs from one. This has beell computed numerically for various coupling constants:

Table [6]

\begin{tabular}{|ccc|}
\hline$\lambda$ & $m / g$ & $1-P(g \bar{q})$ \\
\hline .3325 & 1.6 & $.15(3) \times 10^{-3}$ \\
.5763 & .8 & $.9(1) \times 10^{-3}$ \\
.8158 & .4 & $.30(2) \times 10^{-2}$ \\
.9425 & .2 & $.74(6) \times 10^{-2}$ \\
.9847 & .1 & $.111(3) \times 10^{-1}$ \\
.9961 & .05 & $.1296(5) \times 10^{-1}$ \\
\hline
\end{tabular}

The values and errors were determined using Richardson extrapolation fruni values of $2 H$ ranging from 10 to 24 . The numbers in parentheses give an estima: of the uncertainty in the last digit given by the magnitude in the finai term in the Richardson series. In practice it was possible to restrict the space to at must four-quark states. When computed, six-quark and higher states contributed less than $.1 \%$ to these values at any value of $h$, and so fall below the errors.

As expected, the prohability for higher numbers of guarks is negligible whe:i very massive. Their presence increases steadily with decreasing mass (or increasing coupling) up to $\mathrm{m} / \mathrm{g}$ around .2 , where it appears to levei off at about a per cut.t. 
As will be discussed, results for couplings of $\lambda$ bejond .9425 are probably nut reliable enough to allow any firm conclusions about this region to be drawn. What is surprising is that even as the quarks become massiess, the contribution fron: one extra quark pair remains extremely small, and that that from more quarks is essentially negligible. On the other band, very lighi states, which become massles: wjth the quark mass, do exist in the spectrum which are in fact dominated by higher-Foch states. So states with Jarge numbers of extra qquarks do dominate the low energy spectrum as the quark mass vanishes, but this cannot be detected in the very lightest meson.

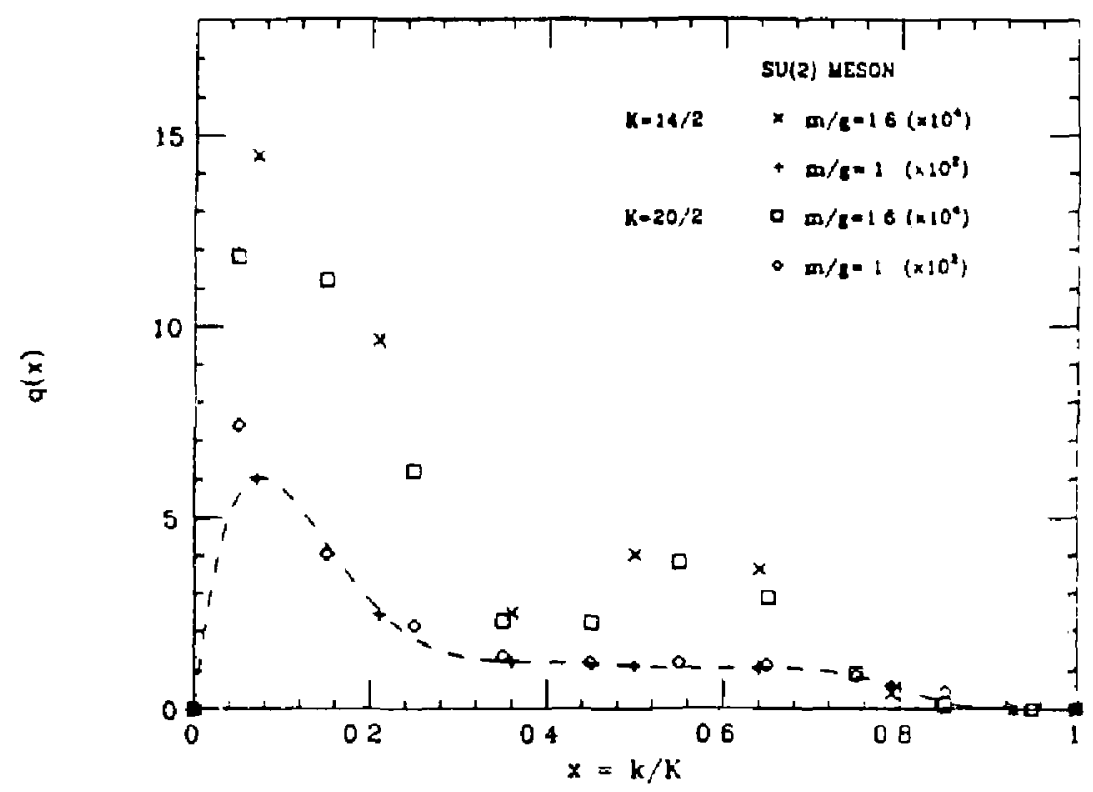

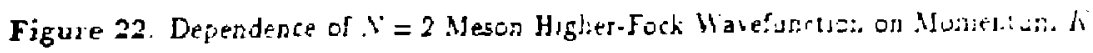

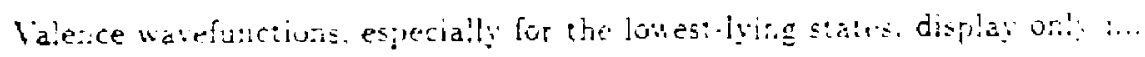


nor sensitivity to the numerical momentum, or resolution, $h$. To esimait the sensitivity of higher-Fock state wavefunctions, the four-quark contribution to thet quark structure function is plotted in Fig. (22) at $2 K$ equal to 14 and 20 for both the weakly and strongly coupled $S U^{\prime}(2)$ meson. The curves are cubic spline fits to the points from $2 K^{\circ}=14$ and are included for ease of comparison.

Note that in order to meaningfully compare these at different $h$, it is necessary to plat the structure function $q\left(x_{k}\right) \equiv J_{i} q_{k}=K^{\prime}\left(b_{k}^{+} b_{k}\right)$, which has a sensible contin. utm limit, ratber than, for example, $\left\langle b_{k}^{+} b_{k}\right\rangle$. Even at these relatively low values for K. the curve shapes and magnitudes are fairly stable. differing by. at most. slightly less than $30 \%$ in the region where $x$ is less than 2 . For larger $I$ the agrcement is much better. For strong coupling, in fact, the points differ by only a fell percent. This suggests that the qualitative features of the higher-Fock wavefunctions, ard in particular their magnitude, can be teliably produced at fairly sma!l $\alpha$.

\subsection{HIGHER-FOCK WAVEFL NCTIONS AT WEAK COLPLING}

The structure functions from the higher-Fock components of the lighilest mesu:n and baryons have displayed a fairly universal, roughly doujle-bump form. For rol. atively weak coupling. lower-lying mesons and baryons are dominated by their valence wavefunctions, and higher-Fock states may be considered perturiarius of these. The structure of the leading bigher-Foch wavefurction, wlich cors. a:t...

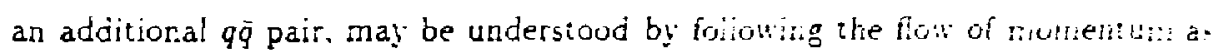
valeace quarks split off extra $q \bar{q}$ pairs. ${ }^{13: i}$

The valence wavefunction for a meson at weak coupling is strongly peaked at

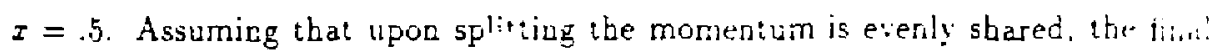



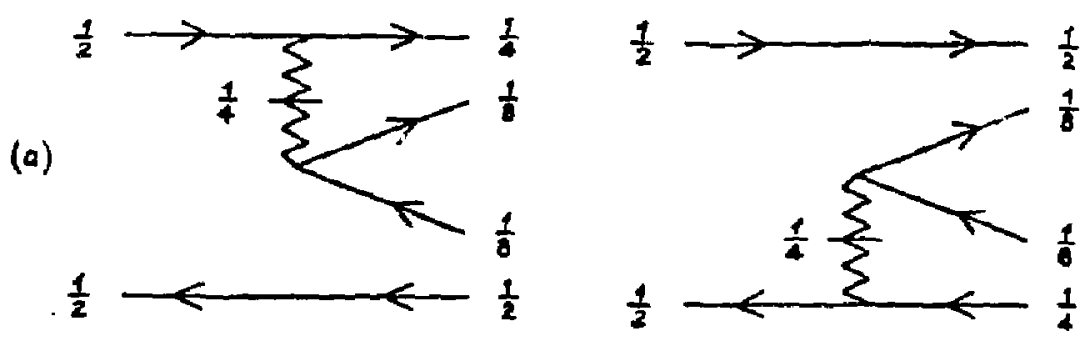

(b)

Figure 23. Momentum Splitling from $q \bar{q}$ to $q \bar{q} q \bar{q}$ Fock States in Meson. (a) Quark Splitting. (b) Antiquark Splitiug.

four-guark wavefunction might be expected to bave momenta distributed accord. ing to Fig. (23). For the quark structure function, the sum of equal-magnitude functions peaked at .25 and .125 (from Fig. (23a)) and .125 and .5 (from Fig. (23b)) would produce a tolal distribution with essentially two peaks, within the resolution of the numerical data. The first would be at roughly .17 (averaging. 25. 125 and .125$)$ with three times the magnitude of the second peak at .5. This struc. ture is evident in Fig. (17) with $\mathrm{m} / \mathrm{g}=1.6$. Apparently; as the coupling constant increases. the spreading of the valence distribution toward larger $x$ is reficcted $i$ : the spreading and increased $x$ of the second peak of the higher. Fock state

For an $S L^{\prime}(3)$ baryon. Fig. (24a) implies a quark distribution witb rouglt?! equal peats at $z=.33$ and $x=.13$ (averaging .083 and .17 ). An antiquark peak should appear at $x=.053$ with about half the height of the quath peaks. Agair. this piovides a fairly accurate description of the weak coupling ( $n / g=i . \epsilon$ : curve in Fig. (15a). For the strongly coupled bayyon, there is not only a spreadirg londat larger $x$, but a factor of greater than ten increase in the iotal probability for find in? an exira go pair: nole the change in scale. Because of the lack of resoliti..... 
(a)

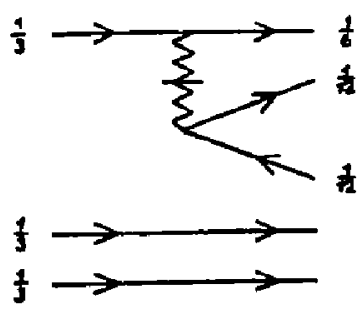

(b)

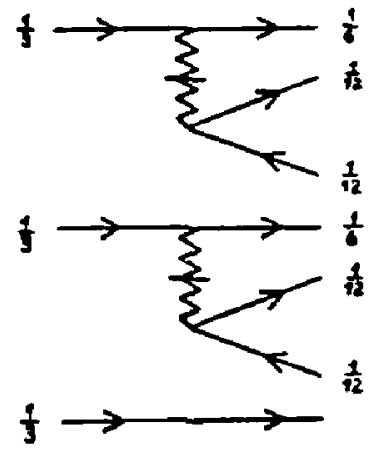

(c)

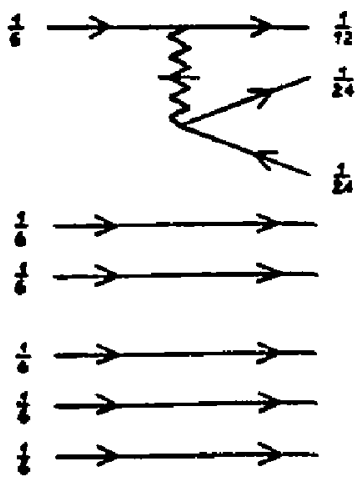

Figure 2a. Momen' um Splitung in $S C^{\prime}$ (3) Baryous (a) Into Fwe-Particle Siate, (b) la:o SeverPartucle Stutc, (c) Baryon Pair into Eight. Partucle State

the pairs of peaks anticipated at small $x$ merge into single peaks. These would presumably be resolved if $K$ were sufficjently increased, providing an additional iest of this general picture.

As a final exercise figs. $(24 b j$ and $(2+c)$ nay be used to undersiand the structure of the seven-quark Foch state for the $.1=3$ baryon with two ad witional git pairs, and the twu-baryon state with one extra pair. Following the previous analys ss and assuming peaks closer than about .1 are unresolved, Fig. (2fb) predicts peaks in the quark structure furstion at $x$ of about .13 and .33 in a ratio of 4 to 1 . Fig. (216) for the two baryon case suggests peaks at .00 and .17 with a ratio of ruglit? 2 to 5 . and also incidentalig a single peak for the antiquarh at or of relative heig:. 1. Within their resolution. Figs. (18b) and (16a) are consistent witi. lias pictune The curves in these plots are drawn to guide the imaginalion. 


\subsection{A PRELIMINARY LOOK AT RENORMIALIZATION}

$Q C D$ in $1+1$ dimensions requires no regularization beyond the subtraction of the vacuum energy: This is not the case in four dimensions, where an appropriate renormalization prescription is essential. In such a scbeme, symmetries such as Lorentz and gauge invariance must either be preserved during discretization or recovered in the continuum limit.

Althougb superfluous, renormalizing $Q C D$ in two dimensions provides an ini. tial example of how this might be approached. Following Ref. [2+j, an ultraviolet cutof is imposed dirrntly on the Fock space. States are restricted to those whose (free) invariant masses satisfy

$$
\sum_{0}\left[\frac{m^{2}+k_{1}^{2}}{x}\right]_{1}<\Lambda^{2}
$$

where the cutoff $\Lambda$ is much larger than the scale of interest. In four dimensions, this regulates both large transverse momentum $k_{\perp}$ and small $x$, while in two dimensions. $k_{-}$is absent. This prescription is Lorentz invariant and analogous to a Pauli-lilla: cutoff in perturbation theory. It will be important in four dinnensions to ensure that such a cutoff is applied in a way that is also gauge-intariant.

As $A$ increases, so does the available resolution. In Table $[i]$, the number of states ir the Fock space with various numbers of parijcles are listed as a furritis. of $A$ for the case where $\lambda=2, B=\dot{u}$ and the morrentum $2 \Lambda=14$. 
Table $[\overline{7}]$

\begin{tabular}{|c|cccc|}
\hline$(\Lambda / m)^{2}$ & $q \bar{q}$ states & $q \bar{q} q \bar{q}$ & $q \bar{q} q \bar{q} q \bar{q}$ & Total \\
\hline 5 & 3 & - & - & 3 \\
10 & 5 & - & - & 5 \\
20 & 7 & 2 & - & 9 \\
30 & 7 & 14 & - & 21 \\
50 & 7 & 28 & 1 & 36 \\
\hline
\end{tabular}

As $A$ increases, the number of states available to describe a $q \bar{q} s y$ stem grows. until limited by the scale of discretization $K$. Also, contributions irom states with increasing numbers of extra $q \bar{q}$ pairs can be resolved. In general. a particular system at a mass scale $M$ should be well-described if $I \gg M$, and $K$ is large enough so as not to cut off the states available at that $\Lambda$.

The running coupling constant $g(A)$ may be defined by requiring some physical quantity, such as a meson mass, to be fixed, presumably by an experiment. At each A. this mass is computed with $k$ in rreasing until contergence. Finally $g$ is adjusted until the computed and physcai quantities match, defining $g(.1)$.

Fig. (25) illustrates this for the case where the physical quantity is an $Y=2$ meson of mass $(M / m)^{2}=6$. The moment um $2 \pi$ is fixed at 26 . which is reascinali: close to convergence, but not quite large enough to produce a smooth curve. gl. behaves as might be expecied for a finite theor: It has a threshold at $(.1 / m)^{2}=i$. where a $q \bar{q}$ pair can first be produced, and begins to turn over and flatter: whern the physical scale $(A / m)^{2}=6$ is reached. As $A$ becomes much larger that this scaie. jt approaches a fixed value. 


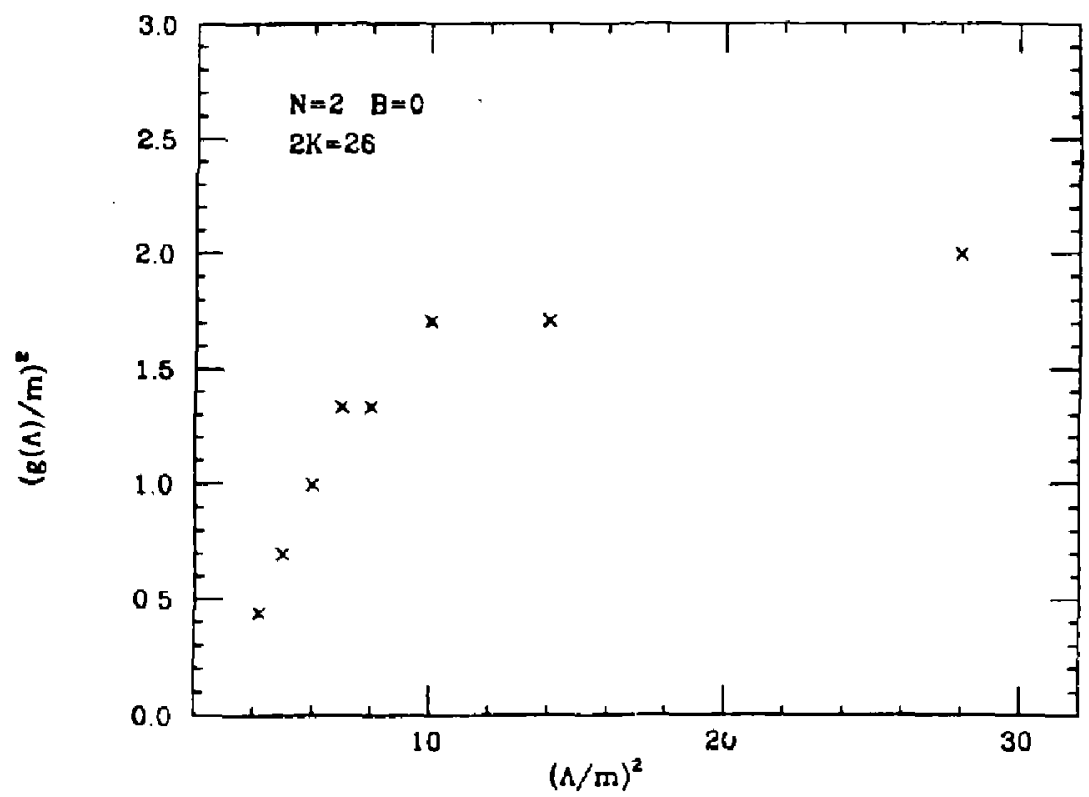

Figure 25. Running Coupling Constant Defined by Holding Fixed the $N=2, B=0$ Neson Mass to $M f^{2} / \mathrm{m}^{2}=6$

\section{ANALYTIC RESUITS}

\subsection{VALENCE MESONINTEGRAL EQLATIONS}

The moment um-space Schrodinger equation

$$
P^{+} P^{-}\left|\rho\left(p^{+}\right)\right\rangle=M^{2}\left|\rho\left(p^{+}\right)\right\rangle
$$

ma: be expressed as an infinite set of coupled integral equations by expantiang $\left|\phi\left(p^{+}\right)\right\rangle$in a Fock state basis and projecting onto the singlet basis states

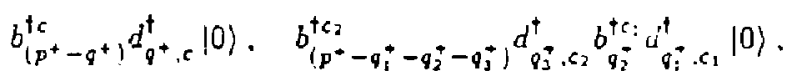

and so on. Quantizing in a box to discretize the momerta and solving Eq. (3.1 b: 
diagonalizing the Hamiltonian is equivalent to evaluating these integral equations on a regularly spaced grid. A great deal of information about the continuum limit of this numerical approach to solving Eq. (3.1) may be extracted by studying these equations. Following Ref. [32], these continuum equations may be derived directly from the discretized Hamiltonian in the $K \rightarrow \infty$ limit. As a bonus, this derivation will demonstrate that the limit is sensible.

As a first step. consider the eigenvalue equation for the meson in the $q \bar{q}$ subspace. The only relevant interactions in the Hamilionian

$$
H=\left(\frac{2 \pi}{L}\right)\left(\frac{\pi \lambda^{2}}{g^{2}}\right) P^{-}
$$

are those which connect $q \bar{q}$ to $q \bar{q}$ :

$$
\sum_{n=\frac{1}{2}, \frac{3}{2}, \ldots}^{\infty}\left[\left(1-\lambda^{2}\right) \frac{1}{n}+\frac{\lambda^{2}}{2} S(n)\right]\left(b_{n}^{\dagger c} b_{n, c}+d_{n, c}^{\dagger} d_{n}^{c}\right)
$$

and

$$
\begin{aligned}
\lambda^{2} \sum_{n}\left[\mathcal{C}_{c_{4} c_{1}}^{c_{3} c_{2}}\left|n_{2}+n_{4}\right|-n_{1}-n_{3} \mid\right. & \\
& \left.\quad-\mathcal{C}_{c_{4} c_{2}}^{c_{2} c_{3}}\left[n_{3}-n_{4} \mid n_{1}-n_{2}\right]\right] b_{n_{4}}^{c_{4}} b_{r_{1}, c_{3}} d_{n_{1}, c_{2}}^{\dagger} d_{n_{1}}^{c_{1}} .
\end{aligned}
$$

The self-energ: $S(n)$ here is defined as

$$
S(k)=\left(\frac{N^{2}-(1-\alpha)}{2 N}\right) \sum_{n=\frac{1}{2} \cdot \frac{3}{2}, \ldots}^{\infty}[[k-n \mid n-k]-[k+n !-k-n]]
$$

and appears in diagonal terms after putting the Hamiltonian into normal order. The instantaneous gluon propagator $[m \mid n]$ and the color tensor $\mathcal{C}$ are discussed in Chapter (1). The parameter $a$ allows $S(N)$ and $[(N)$ to be treated simultaneously: for $S U(N), \alpha=0$ and $\alpha=1$ for $(" N)$. 
The eigenstate $\left|\phi\left(K^{\circ}\right)\right\rangle$ may, in general, be expanded in this subspace

$$
\left|\phi\left(K^{\prime}\right)\right\rangle=\sum_{n=\frac{1}{2}, \frac{3}{2}, \cdots}^{h-\frac{1}{2}} \phi_{n} b_{h^{\prime}-n}^{\dagger c} d_{n, c}^{\dagger}|0\rangle
$$

Sandwiching the eigenvalue equation

$$
H|\phi\rangle=\frac{M_{0}^{2}}{\kappa^{\prime}}|\phi\rangle
$$

$\because$ ith $\langle 0| d_{l}^{\prime} b_{K^{\prime}-\ell, c^{\prime}}$ results in the discrete equation

$$
\begin{aligned}
& {\left[N\left(1-\lambda^{2}\right)\left(\frac{1}{\ell}+\frac{1}{R-\ell}\right)+\frac{1}{2} \lambda^{2} \Lambda[S(\theta)+S(\lambda-\theta)]\right] o_{\ell}} \\
& +\frac{1}{2} \lambda^{2} \sum_{n=\frac{1}{2}, \frac{3}{2}, \ldots}^{k-\frac{1}{2}}\left[-\left(N^{2}-(1-a)\right) \frac{1}{(l-n)^{2}}+\alpha N \frac{1}{K^{2}}\right] o_{n} \\
& =\frac{M_{0}^{2} N}{h^{\prime}} \phi_{i} \text {. }
\end{aligned}
$$

$M_{0}^{2}$ is the numerical mass squared which appears in the program and is $1 t^{2} /\left(m^{2}+g^{2} / \pi\right)$ in dimensional units. The singularity at $n=c$ is excluded by tand in the definition of $H$.

Ling the identity ${ }^{|32|}$

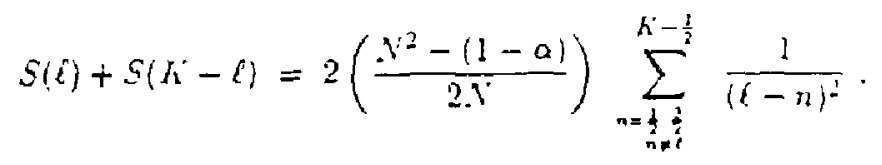

defining the momentum fractions $x=E / K$ and $y=n / h$, converting of into $1 ! \cdot, \cdot$ continuum wavefunction oi $x$ ), and reintroducing dimensional patameters produses 
in the continuum limit $K \rightarrow \infty$

$$
\begin{aligned}
m^{2}\left(\frac{1}{x}\right. & \left.+\frac{1}{1-x}\right) \phi(x)-\frac{g^{2}}{\pi}\left(\frac{\Lambda^{2}-(1-\alpha)}{2 N}\right) f_{0}^{1} d y \frac{\phi(y)-\phi(x)}{(y-x)^{2}} \\
& +\frac{g^{2} \alpha}{2 \pi} \int_{0}^{1} d y \phi(y)=M^{2} \phi(x) .
\end{aligned}
$$

The principal value prescription is fixed by the infrared regularization of the discrete Hamiltonian before the $H \rightarrow \infty$ limit is taken. Specifically.

$$
\int_{0}^{1} d y \equiv \lim _{l \rightarrow 0} \int_{0}^{x-c / 2}+\int_{z+c / 2}^{1} d y
$$

where $\epsilon=1 / K^{\circ}$. With this prescription, Eq. (3.10) is well-defined, lending confidence that the regularization adopted for numerical calculations has a sensible continuum linit.

Although this equation is in a severely restricted space, a great deal of informaticn about the continuum limit of the model may be extracted 'sum: it. bevond the observation that such a limit exists. For example, if the quar'i mass $m$ is set to zero, $\phi(x)=1$ i clearly an eigenfunction, with $A S^{2}$ equal to zero for $S[(\lambda)$ $(\alpha=0)$. For $U(Y)(a=1)$, the presence of the extra term from $q \bar{q}$ annihilation. $\left(g^{2} / 2 \pi\right) \int_{0}^{1} d y \phi(y)$, is related to the $l(1)$ anomaly and gives these mesons a ma:.. squared of $M^{2}=g^{2} / 2 \pi$. Eq. (3.10) thus reproduces by inspection the masses a:tu? wavefunctions for $S L^{\circ}\left(N^{\prime}\right)$ and $U(N)$ mesons in the $\mathrm{m} / \mathrm{g} \rightarrow 0$ limi. In fac!. for $U(1)$ (the Schwinger model). it yields the exact mass for the only particle in th." theory ${ }^{[3: ! 3]}$ Because of the Fock space resirjution, however, this equation camin: 
describe states which contain pairs of these mesons. nor the higher Sock crntent of individual mesons.

The equation also makes apparent the possibly singular nature of the $m=0$ limit. The free Hamiltonian, which is proportional to $\mathrm{m}^{2}$. diverges for quarks sarrying zero momentum, forcing wavefunctions to waish at $x_{1}=0$. For $\mathrm{m}^{2}$ jdentically zero, this restriction disappears; it is not necessarily true that the exact solutions at $m=0$ are related to those for sma!!, but finite, $m$.

Interestingly; this equation contain: all of the information necessary to conpute the spectrum of mesons in the opposite, nonrelativistic, limit where sim $\rightarrow 0$. In this limit, the large mass of the quark dynamically rest 'cts the statrs to quarkantiquark pairs. The equation and its solutions in this limit, as well as that for haryons, will be discussed later in this chapter.

Finally, in the large- $N$ limit $\left(\Lambda^{*} \rightarrow \infty\right.$, with $g^{2} \Lambda^{*}$ fxed $)$ retaining only terms of order $g^{2} N$ in $\mathrm{Eq}$. (3.10) reproduces the equation derived iu Hef. [2]', by sul, aring the leading planar diagrams which contribute to the valence meson propagator. In this limit, the $\{(N)$ and $S U(N)$ cases are equivalent. The extra ierm in the $(Y, Y)$ equation which produces the massive Srliwinger bosons as $m / g \rightarrow 0$ is proportional to $g^{2}$ and so is suppressed by one powir of $\Lambda$. As a result. when $\mathrm{m} / g \rightarrow 0$. the large- $\mathcal{N}$ limit neg!ects the leading contribution to the mass of these mesons. and they become massless as in $S L^{\prime}(X)$.

It should be noted that some references, beginning with Ref. [2! disciss a finite renormalization of the quart mass $m^{2} \rightarrow m^{2}-\left(g^{2}, 1 / 2-\right)$, to order $g^{2} .1$ and $i$. my conventions. In these references, a slightly different principal value prescriptis: 
is used. With that prescription, the second term in the integrand of

$$
-\left(\frac{g^{2} N}{2 \pi}\right) \int_{0}^{1} d y \frac{\phi(y)-\phi(x)}{(y-x)^{2}}
$$

in Eq. (3.10) yields

$$
-\left(\frac{g^{2} N}{2 \pi}\right)\left(\frac{1}{x}+\frac{1}{1-x}\right) \phi(x)
$$

which finitely renormalizes $m^{2}$. This is simply a rearrangement of terms and the equations are identical. Incidentally, in the space of color singlets, this renormal. ization is of no direct physical consequence. In perturbation theory, it alters only. the gauge-dependent quark propagator. ${ }^{\text {(Jo) }}$

\subsection{VALENCE BARYON INTEGRAL EQUATIONS}

The continuus limit integral equation for the baryon, restricted to the ninimum $x$ valence quarks. may be derived in the same manner as the meson equation. The relevant terms in the discretized Hamiltonian are

$$
\left.\sum_{n=\frac{1}{2}, \frac{3}{2} \ldots}^{\infty}\left(11-\lambda^{2}\right) \frac{1}{n}+\frac{\lambda^{2}}{2} S(n)\right) b_{n}^{\dagger c} b_{n, c}
$$

and

$$
-\frac{\lambda^{2}}{2} \sum_{n} \mathcal{C}_{c_{1} c_{3}}^{c_{1} c_{2}}\left[n_{2}-n_{4} \mid n_{1}-n_{3}\right] b_{n_{4}}^{\dagger c_{1}} b_{n_{3}}^{\dagger c_{1}} b_{n_{2}, c_{2}} \dot{b}_{n_{1}, c_{2}}
$$

Consider first the case where $N=3$ : the generalization to arbitrary $N$ will fo...n. 
A color singlet valence baryon state will have the form

$$
\left|\phi_{B}\left(K^{*}\right)\right\rangle=\sum_{n} \delta_{K^{\prime} \cdot \Sigma n} \phi_{B}\left(n_{1}\right) \epsilon_{\varepsilon_{1} c_{2} c_{2}} b_{n_{1}}^{\dagger c_{1}} b_{n_{3}}^{\dagger c_{2}} b_{n_{3}}^{\dagger c_{3}}|0\rangle
$$

As was done for the meson, the Schrodinger equation

$$
H\left|\phi_{B}\right\rangle=\frac{M_{0}^{2}}{K}\left|\phi_{B}\right\rangle
$$

is projected onto the singlet state

$$
\epsilon_{c_{1}^{\prime} c_{2}^{\prime} c_{3}^{\prime}} b_{l_{1}}^{t c i} b_{l_{2}}^{t c i} b_{l_{3}}^{t c_{3}^{\prime}}|0\rangle
$$

where $\sum l_{1}=K$. Contracting color and moment um indices and taking the continuum limit $K \rightarrow \infty$ converts Eq. (3.1i) into

$$
\begin{aligned}
& \sum_{i=1}^{N} \frac{m^{2}}{x_{1}} \phi_{B}(x)-\frac{g^{2}}{\pi}\left(\frac{N+1}{2 \lambda}\right)[ \\
& \int_{d_{1 / 2}}^{x_{1}} \frac{d s}{s^{2}}\left(O_{B}\left(x_{1}-s, x_{2}+s, x_{3}\right)+O_{B}\left(x_{1}-s, x_{2}, x_{3}+s\right)-(N-1)_{B}(x)\right) \\
& +\int_{d_{1} 2}^{z_{2}} \frac{d s}{s^{2}}\left(\phi_{B}\left(x_{1}+s_{1} x_{2}-s_{1} x_{3}\right)+\phi_{B}\left(x_{1}, x_{2}-s_{1} x_{3}+s\right)-\left(\Lambda-1 \rho_{B}(x)\right)\right. \\
& \left.+\int_{C / 2}^{x_{3}} \frac{d s}{s^{2}}\left(O_{B}\left(x_{1}+s_{1} x_{2} \cdot x_{3}-s\right)+\varphi_{B}\left(x_{1} \cdot x_{2}+s \cdot x_{3}-s\right)-\left(x-1 \varphi_{O_{B}} x_{1}\right)\right)\right] \\
& =M^{2} O_{B}(x) \text {. }
\end{aligned}
$$

Here $\dot{\phi}_{B}(x) \equiv Q_{B}\left(x_{1}, x_{2}, x_{3}\right)$ and $\sum_{1} x_{i}=1$. The $\epsilon / 2$ cutoff for small $x$ is the samie principal value prescription employed in the meson equation. ${ }^{[3]^{3}[]^{3}: 1}$ This equation 
has been derived both by Feynman diagrams in Ref. [33] and by means of an equivalent string theory is Ref. [34], and has beea studied oumerically in Ref. [35].

In this form the equation is readiy extended to arbitrary $\Lambda$. The wavefunction takes $N$ arguments, there are $N$ rather than 3 integrals, and in the $i^{\text {th }}$ integral there are $(N-1)$ terms in which momentum is transferred consecutively from the $i^{\text {th }}$ quark to the remaining $N-1$ quarks.

\subsection{MESON WAVEFUNCTION ENDPOINT BEHAIIOR}

The asymptutic behavior of the valence $S L(N)$ meson wavefunction when onc quark carries momentum fraction $x \sim 0$ may be determined using the contin $n$ integral equation Eq. (3.10). ${ }^{|38|}$ The behavior for valence barvons and higher-I it states may also be computed from the relevant integrai equations, as will be shuwn later. The wavefunctions in this limit will provide a great deal of information about continuum solutions, particularly when $\mathrm{m} / \mathrm{g}$ is small, or for form factors, when $Q^{2}$ is large. In addition, it will be employed to determine the dependence of numerical results on $h^{\circ}$.

Assuming the form $\varphi(x) \sim c x^{a}$ as $x \sim 0$. where $c$ is an undetermined constant. Eq. (3.10) becomes

$$
m^{2} c x^{2-1}-\frac{g_{x}^{2}}{\pi} \int_{0}^{1} d y \frac{o(y)-c g^{a}}{(y-s)^{2}} \sim 0
$$

witi:

$$
g_{i}^{2} \equiv g^{2}\left(\frac{\gamma^{2}-1}{2 X^{\prime}}\right)
$$


The integrai in Eq. (3.20) must ireroduce an extra power of $1 / x$ to balance the kinetic term. This will originate in the $y \sim I$ region, where $\phi(y) \sim c y^{a}$. Defining the integration variable $u \equiv y / x-1$ in the region $y \in[0, x-\epsilon]$ gives, to leading order in $x$

$$
\int_{0}^{x-c} d y \frac{y^{a}-x^{a}}{(y-x)^{2}}=x^{a-1} \int_{-1}^{-e / x} d u \frac{(1+u)^{a}-1}{u^{2}},
$$

which possesses the appropriate power of $x$. Repeating for $y \in[x+\epsilon, 1]$ and taking $c \rightarrow 0$ prior to $x$ gives for Eq. $(3.20)$

$$
\int_{-1}^{\infty} d u \frac{(1+u)^{a}-1}{u^{2}}=\frac{\pi m^{2}}{9 \pi} .
$$

The integral is evaluated in Appendix B, reproducing the equation in Ref. [21] (for large $\left.N^{*}\right)$

$$
1-(a \pi) \cot (a \pi)=\frac{\pi m^{2}}{g_{i}^{2}}
$$

Yith $a \in(0,1)$. Note that this determines, up to a coefficient, the same asymptotic small- $x$ behavior for all $g \bar{q}$ wavefunctions, including excited states. It depends vit the parameter $\mathrm{m} / \mathrm{g}$ but is independent of each state's mass. The coefficient $c$. however, may vary from state to state.

The exponent a determined implicitly by Eq. (3.2.) monotonirally increacefrom 0 to 1 as $\mathrm{m} / \mathrm{g}$ runs from 0 to $\propto$. For strong coupling (small mass)

$$
a_{(m / g \sim 0)} \sim\left(\frac{3}{\pi}\right)^{1 / 2}\left(\frac{m}{g . n}\right)
$$

while for weak coupling (Jarge mass)

$$
a_{\{g / m \sim 0 \mid} \sim 1-\frac{1}{\pi}\left(\frac{g: x}{m}\right)^{2} .
$$


The reasonable approximation for the lightest meson wavefunction,

$$
\phi(x) \propto x^{1}(1-x)^{a},
$$

provides a fairly accurate mass expectation val e at strong coupling. As $m / g \rightarrow 0$. the exponent $a$ approaches zero, and $\phi(x) \sim 1$ everywhere except for an increasingly narrow region near the endpoints, where it decreases sharply to zero. This behavior will mate accurate numerical calculations based on a regular grid increasingly. difficult. For weaker coupling the wavefunction grows less drastically from zero at its endpoints, while the peati at $x=1 / 2$ becomes more promineut. Finally. it should be mentioned that there do exist other possible asymptotic forms for $\boldsymbol{x} \sim 0$ which may be generated from those above by taking $n$ derivatives with respect to $a$. These go as $x^{a} \log ^{2} x$, where $a$ is the same function of $m / g$ as before. Expectation values for $1 I^{2}$ which have been computed with $\phi(x) \times x^{a}(1-x)^{a} \log ^{n}[x(1-x)]$ ] consistent]y higher than for those discussed above, and the previous form will be assumed.

\subsection{BARYON WAVEFLNCTION ENDPOINT BEHAIIOR}

Just as for the meson, the asymptotic behavior of the baryon wavefunction in the valence approximation as one argument approaches zero may be extracted from the continum Eq. (3.19). The case where $\mathrm{N}=3$ will be considered: atrin. the extension to general $N$ is simple.

In the limit where $r_{1} \rightarrow 0$ while the other $r_{\text {a }}$ are fixed. the form for $\tilde{o}_{B}$

$$
O_{B}(x) \sim x_{1}^{a} \dot{O}_{D}\left|x_{2} \cdot x_{3}\right|
$$


is taken as an ansatz. The kinetic term is then

$$
H_{\circlearrowleft} \phi_{B} \sim m^{2} x_{1}^{a-1} \dot{\phi}_{B}
$$

Also, the first integral in Eq. (3.19), after the change of variables $s=-x_{1} u$, gives, to leading ordei in $I,(N-1)$ copies of

$$
-\frac{g^{2}}{\pi}\left(\frac{N+1}{2 N^{\prime}}\right) x_{1}^{a-1} \tilde{\phi}_{B} \int_{-1}^{0} \frac{d u}{u^{2}}\left[(1+u)^{a}-1\right] .
$$

The remaining integrals contain $(N-1)$ terms, one from each integral, in whoh momentum is exchanged with the first quark; that is, $x_{1}$ appears in the combination $x_{1}+s$. Defining $s=x_{1} u$, these produce

$$
(N-I)\left[\frac{-g^{2}}{\pi}\left(\frac{N+1}{2 \Lambda^{3}}\right) x_{1}^{a-1} \dot{\phi}_{B} \int_{0}^{\infty} \frac{d u}{u^{2}}(1+u)^{a}\right],
$$

while those remaining partially cancel and contribute a term of the same form but with $(1+u)^{a}$ replaced by -1 .

The net result is that as $x_{1} \rightarrow 0$. a must satisfy

$$
\int_{-1}^{\infty} \frac{d u}{u^{2}}\left[(1+u)^{a}-1\right]=1-(a \pi) \cot (a-1)=\frac{\pi m^{2}}{9 !} .
$$

This is the same equation as for the meson wavefunction in the same limit. The as!mptotic small- $x$ behavior of wavefunctions appears to be universal: this equatiun is valid for mesons and for baryons with any number $l$ of quarks. This result is unaffected by permitting bigher-Fock states in at leas: the meson wavefunction. a will be shown. and is probably true in general. 
Finally, this limit was defined such that all but one argument are held fixed. If more than one $I_{3}$ is taken to zero simultareously, the behavior is more complicated. For example, for Eq. (3.19), letting $x_{1}$ and $x_{2}$ approach zero holding $x_{2} / x_{1}=\sigma$ fixed and assuming

$$
\phi_{B} \sim s_{1}^{u} x_{2}^{b} \dot{\phi}_{B}
$$

gives the condition

$$
\begin{aligned}
{\left[m^{2}-r(1-(a \pi) \cot (u \pi j)]\right.} & +\frac{1}{\sigma}\left[m^{2}-r(1-(b \pi) \cot (b \pi))\right] \\
& -\gamma \int_{-1}^{\sigma} \frac{d u}{u^{2}}\left[\left(1+u i^{a}(1-u / \sigma i)^{b}-1\right]=0 .\right.
\end{aligned}
$$

with

$$
\gamma \equiv \frac{g^{2}}{\pi}\left(\frac{N+1}{2 Y}\right)
$$

The general behavior of wavefunctions when one argunicnt approaches one and the rest approach zero would be particularly useful for determining the behaviot of form factors at large momentum transfer: this wiil not be at tempted here. The specific case of the large- 1 vaience meson is discussed in Ref :15;

\subsection{ESTIMATING NUMERICAL ERRORS}

In the continum limit, the problem of diagonalizing $P^{-}$ma: Lir ca-t ir: $:$t. form of an infinite set of coupled integral equations. From the derivation of th...... equations in the previcus sections, it is clear that diaguadizats $P^{-}$in the dierre...

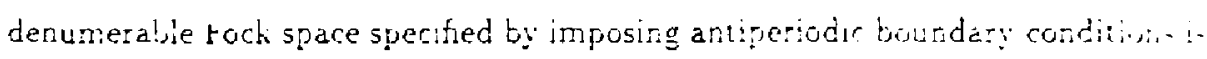


equivalent to solving these equations numerically, with the integrals evaluated on a regularly spaced grjd. For example, in the valence meson equation. Eq. (3.10),

$$
\sum_{n=\frac{1}{2+\frac{1}{2}}}^{h-\frac{1}{2}} \frac{\phi_{n}-\phi_{l}}{K\left(n / h-\ell / h^{2}\right)^{2}} \underset{h \rightarrow \infty}{\rightarrow} f_{0}^{1} d y \frac{\phi(y)-\phi(x)}{(y-x)^{2}}
$$

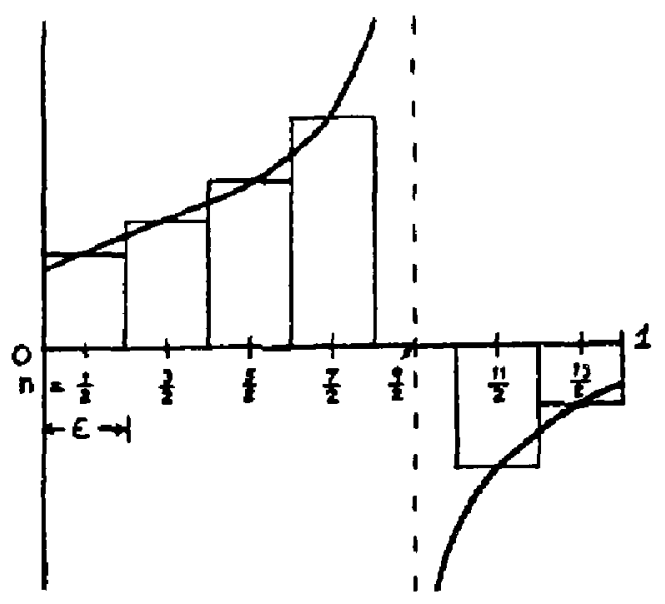

Figure 20. Numerica! Aproximalıon of Priticipal- Yalue Regulated Integral

For finite $\dot{K}$, the sum approximates the integral by summing rectangles of width $\epsilon=1, h$ as in Fig. 26. The contribution from $n=\ell(=9 / 2$ in this figure $\mathrm{i}$ is excluded. The error typically induced by approximating the integral of fiy from $y=a$ to $b$ in this way is

$$
\left.\left.\frac{\epsilon^{2}}{12} \mid f^{\prime}(b)-f^{\prime}(a)\right]+\mathcal{O}_{1} e^{3}\right)
$$

This is aolmally not a good scheme for approximating an integra!. and mat: laster methods exist. The case considered above, bomever, is not even nor:ai: 
First, because $\dot{\phi}(x) \propto x^{a}$ as $x \sim 0$, with $a \in(0,1)$, the derivative of the integrand appearing in the error estimate contains terms which diverge at the endpoints as $x^{a-1}$. Second, the singularity regulated by the principal value induces an additional error when discretized.

To determine the discretization error in the valence meson integral, consider the regions $[0, \epsilon]$, and $[1-\epsilon, 1]$ about the endpoints, and $[\epsilon, x-\epsilon / 2]$, and $[x+\epsilon / 2,1-\epsilon]$ near $x$, separately; $x$ will be assumed as fixed and finite.

First, computing the integral (with $\phi(y) \sim c y^{a}$ )

$$
\int_{0}^{2 c} d y \frac{c y^{2}}{(y-x)^{2}}
$$

and subtracting that approximited by sampling at the first grid point at $y=\epsilon / 2$. produces a leading error of

$$
\frac{2 \varepsilon}{x^{2}}\left(\frac{2^{a}}{1+a}-1\right) \epsilon^{1+a}
$$

with higher terms of order $\epsilon^{n+a}$, with $n>1$. The contribution from the term with $-\phi(x)$ in the numerator produces errors of order $\epsilon^{n}$ with $n \geq 3$ in this region. The region near $y \sim 1$ should produce the same order errors. In $[\epsilon, x-\epsilon / 2]$ and $[x+\epsilon / 2,1-\epsilon]$ the integrand and its derivatives are firite and the usual error estimate applies. The endpoints neighboring the excluded region around $y=x$ contribute errors of order $\epsilon^{n}, n \geq 3$ to Eq. (3.3.3). Those near $y=0$ and 1 yielt? errors of order $\epsilon^{n+a}$ and $e^{n+1}$, with $n \geq 1$.

Finaly, eliminatirg the point from the sum in Eq. (3.32) where $n=t$ (that is. $y=I$, effectively excludes the region $[x-\epsilon, x+\epsilon]$ from the integration over $y:$ icf. 
Fig. (26)). The error induced is

$$
\int_{x-c}^{x+c} d y \frac{\phi(y)-\phi(x)}{(y-x)^{2}}
$$

where $\epsilon$ is fixed at $1 / h$ while the principal value limit is taken. Assuming $\phi(y)$ is sufficiently smooth and expanding around $x$ gives for Eq. (3.36)

$$
\phi^{\prime \prime}(x) \epsilon+\frac{1}{36} \phi^{\prime \prime \prime \prime}(x) \epsilon^{3}+\ldots
$$

Although in the limit $K \rightarrow \infty(\epsilon \rightarrow 0)$ the discrete infrared regularization employed reduces to a well-defined principal value prescription, it reduces at a leisurely pact: for finite $K$ it is the source of the leading $\mathcal{O}(\epsilon)$ error.

Combining contributions from these varjous terms gives a series in cor the total error in Eq. (3.32) of

$$
c_{1} \epsilon+c_{2} t^{1+a}+c_{3} t^{2}+c_{4} t^{2+a}+\ldots
$$

in contrast with terms of orde: $\epsilon^{n}, n \geq 2$ whirh would ocrur were ihe integra:. analytic

Because the endpoint behavior $a$ is apparent!y general. physical quantities $M$, such as meson and baryon masses. measured at finite but sufficiently large $K$. should behave as

$$
M(1 / K)=. M(0)+\frac{c_{1}}{K^{2}}+\frac{c_{2}}{K^{2}+a}+\frac{c_{3}}{K^{2}}+\frac{c_{4}}{h^{2}+a}+\cdots .
$$

with $11(0)$ the continuum limit. The leading error is of order $1 / K$, so rhat $1 / 1 / K$ i converges painfully slowly. The discretization schenle used to quantize tbis t!atory then ends up effectively evaluating integrals in a way which is simple bu: 
inefficient. However, knowing Eq. (3.39), this may be turned to advantage, and convergence may be improved significantly by Richardson extrapolation. In this method, $M(1 / K)$ is computed at $n$ different finite values of $K$, to which the first $n$ coefficients of the saries are fit. This technique is commonly applied to improve numerical calculations of integrals, although the dependence of the series behavior on the coupling and mass through the exponent $a$ is peculiar to this case. The error to the fit of $M(0)$ is of the order of the term $n+1$. A rough estmate of the error in this procedure is given by the last term retained. This technique was uscd in the results previously quoted to allow for a meaningful comparison with other techniques, and to give some idea of their uncertainties.

\subsection{ESTIMATE OF NECESSARY RESOLUTICN}

In order to know when this extrapolation procedure may be reliably applied. it is useiul to know how large $K$ must be before results begin to exhibit the behavior in $\mathrm{Eq} \cdot(3.39)$.

The approximate valence wavefunction for the lightest mesun

$$
\phi(x) \propto x^{a}(1-x)^{a},
$$

where $a$ is defined in Eq. (3.22), provides a model for estinating the size of $l i$ needed for reasonable numerical results. This is especially important to know in the case of strong coupling (small quark mass). As has been seen, a becomes sna!! and $O(x)$ turns over sharply at jts endpoints. $h^{*}$ will most likely be large enongh when it allows these regions to be adequately sampled. 
When discretized. $x_{k}=k / K$, where $k$ is the numerical quark momentum. If the first point in $x$ is required to be small enough to sample $\phi(x)$ before the point where $\phi(x)$ reaches half jts maximum, then

$$
\text { ii } 2 \frac{1}{2 x_{h \max }} \text {, }
$$

with

$$
x_{h \max }=\frac{1}{2}-\frac{1}{2}\left(1-\frac{1}{2^{1 / a}}\right)^{1 / 2} .
$$

The minimum $K$ estimated in this way is listed in Table $\{\delta\}$ as a function of $\lambda$ For A from 2 to 4 .

\begin{tabular}{|c|c|c|c|c|}
\hline$\lambda$ & $\mathrm{m} / \mathrm{g}$ & $\begin{array}{l}K_{\min }: \\
N=2\end{array}$ & 3 & 4 \\
\hline .3325 & 1.6 & 8 & 8 & 10 \\
\hline .5763 & .8 & 10 & 12 & 15 \\
\hline .8158 & .4 & 20 & 30 & 50 \\
\hline .9425 & .2 & 90 & 250 & 500 \\
\hline 9847 & .1 & 2000 & 15000 & $7 \times 10^{4}$ \\
\hline .9961 & .05 & $10^{6}$ & $5 \times 10^{7}$ & $10^{9}$ \\
\hline
\end{tabular}

Table [s]

These estimates are probably overly conservative for computations of quantitilus which are not sensitive to the endpoint behavior of the wavefunctions. It docs. however, indjcate that aumerical precision becomes increasingly difficult as the coupling strength grows; the $h$ considered in actual computations are generi?!: below 50 . so results for $\lambda>9125$ should be eyed suspiciouij: 

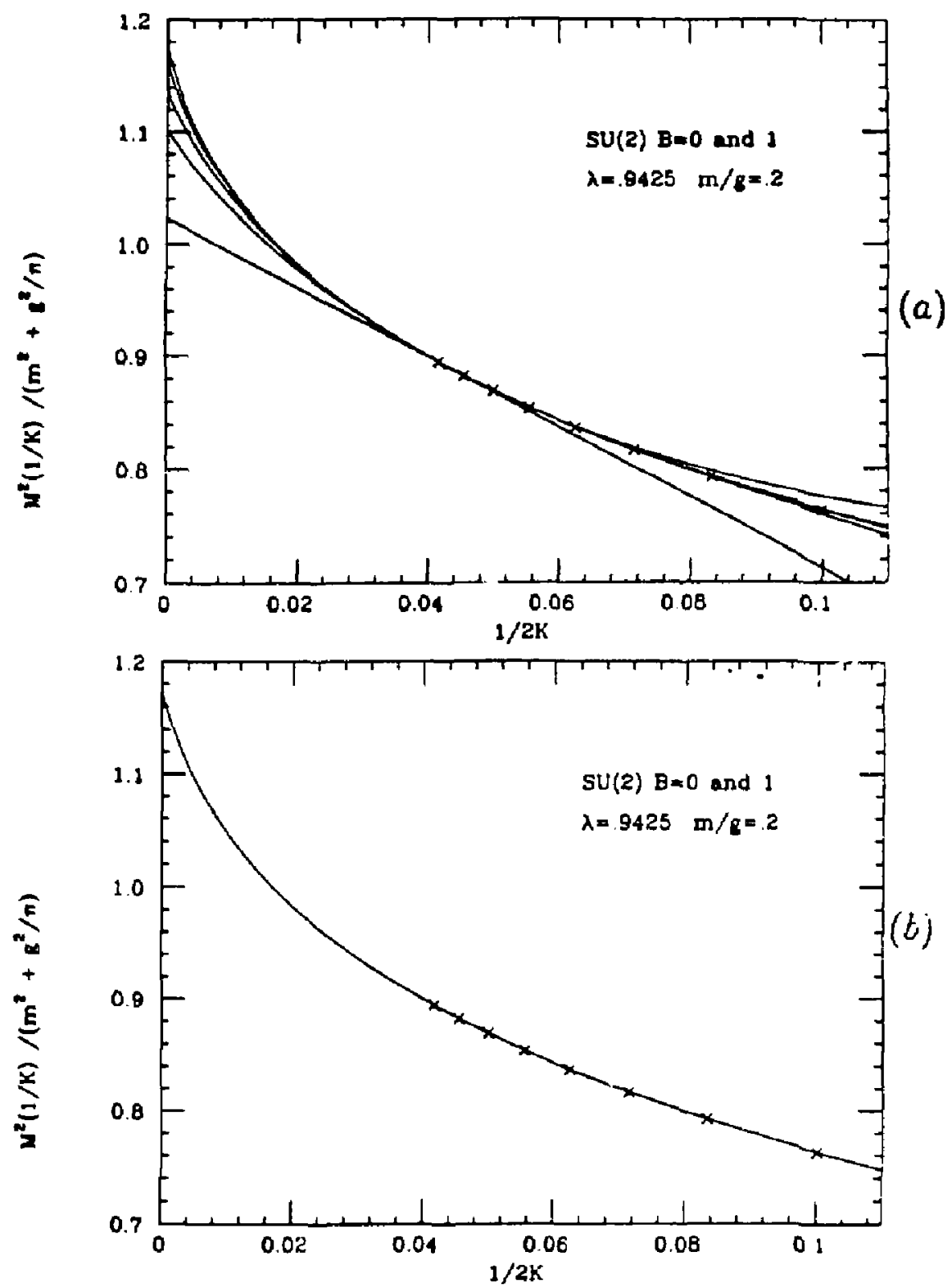

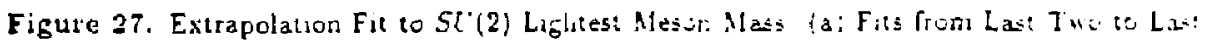
Six Points, (b) Fit :- Last Six Points isclated 
Fig. (27) provides an example of a fit of this kind. Eight values for the lightest meson mass-squared $M_{0}^{2}$ in units of $\mathrm{m}^{2}+g^{2} / \pi$ are plotted versus $1,2 K$. where $2 H$ ranges from 10 to 24 . Here $N=2$ and $\lambda=.9425$, cortesponding to $m / g=.2$. For $S U(2)$, the lightest meson and baryon masses are identical at all values of $h$, so these apply as well to the baryon. Also, the Frock space was restricted to four quarks; the resulting error is negligible for this case.

These points are used to determine the coefficients in the series expected to describe the behavior of $M_{0}^{2}$ as a function of $1 / 2 K$, as in Eq. (3.39). For this case. $a=.222$ for $\lambda=.9425$. This function is plotted in Fig. (2ia) for the cases where the left-most $n$ poivis are used to determine the first $n$ coefficients in Eq. (3.39). and $n$ ranges from two to six. Fig. (27b) isolates the six-term series. The vertical intercept is $M_{0}^{2}(0)$.

From these plots, it is clear that extrapolation substantially improves, or at least alters, the best measured value for $M_{0}^{2}$ at $2 K=24$. In fact, the $\lambda$ in this case is probably close to the upper limit for which computations at this $K$ are seliable. Is spite of the rather large extrapolation, the curves are both smooth. that is devoid of oscillations between points, and stable as the number of included points increases. Also, the curve in Fig. (27b) is fit to only the six points nearest the continuum, but nevertheless runs through the next two points to the right These suggest that this fit is reasonably reliable.

The value of the last lerm retained in the series (computed at the smallest value of $K$ used in the fit) provides an indication of the error. For $n=2$ to 6 , these are $.14, .42 .080, .39$, and .073 , respectively: These error estimates are admiltedly. crude; a much better method could certainly be devised. This would probably be 
warranted if these calculations were being compared with actual physical (that is. four-dimensional) quantities.

\subsection{HIGHER-FOCK EQUATIONS: ENDPOINTS AND ERRORS}

Because the endpoint behavior of meson and baryon wavefunctions, derived above from integral equations in the valence approximation. play an important role in botb analytic and numerical results, it is importan to know whlat effect the inclusion of higher-Fock components might have.

Just as in the valence approximation, integral equations which incorporate higher-Fock wavefunctions may be derived from the discretized Hamilionian b: contracting the Schrodinger equation with appropriate color singlets. For example. for $B=0$. contracting Eq. (3.17) on the left with a $q \bar{q}$ singlet at fixed momentum and taking $K$ (or $L$ ) to infinity produces an integral equation which incorporates the $q \bar{q}$ and $q \bar{q} g \bar{q}$ meson wavefuuctions. Because $H$ cas change particle number by at most two. no others are involved in this case.

The result is the complete equation for $\phi_{2}(x) \equiv \emptyset_{2}(x, 1-I)$ : 


$$
\begin{aligned}
M^{2} \phi_{2}(x) & =m^{2}\left(\frac{1}{x_{1}}+\frac{1}{x_{2}}\right) \phi_{2}(x) \\
& -\frac{g^{2}}{\pi}\left(\frac{N^{2}-a}{2 N}\right) \int_{0}^{1} d y \frac{\phi_{2}(y)-\psi_{2}(x)}{\left(y-x_{1}\right)^{2}}+\frac{g^{2}}{\pi}\left(\frac{1-a}{2}\right) \int_{0}^{1} d y \phi_{2}(y) \\
& -4 \frac{g^{2}}{\pi} \int_{0}^{1}[d y] \phi_{4}\left(x_{1}, y_{2} ; y_{3}, y_{4}\right)\left[\left(\frac{N^{2}-\alpha}{2 N}\right) \frac{1}{\left(x_{2}-y_{4}\right)^{2}}-\left(\frac{1-a}{2}\right) \frac{1}{\left(x_{2}-y_{2}\right)^{2}}\right] \\
& +4 \frac{g^{2}}{\pi} \int_{0}^{1}\left[d y \phi_{4}\left(y_{1}, x_{2} ; y_{3}, y_{4}\right)\left[\left(\frac{N^{2}-\alpha}{2 N^{2}}\right) \frac{1}{\left(x_{3}-y_{3}\right)^{2}}-\left(\frac{1-a}{2}\right) \frac{1}{\left(x_{1}-y_{1} !^{2}\right.}\right] .\right.
\end{aligned}
$$

Here $[d y\}$ is short-hand for $\prod_{1} d y_{2}$, with the $y_{1}$ chose appearing in $\phi_{4}$, multiplied by a delia function which restricts all the arguments of $\dot{\phi}_{4}$ to sum to one. The variables $x_{1}$ and $I_{2}$ are the fractional momenta of the $q$ and $\bar{q}$ which contracted Eq. (3.17), with $x_{1}+x_{2}=1$. Finally, the concinuum $n$-particle wavefunctions $o_{n}\left(I_{1}\right)$ are constructed from the discrete $\phi_{n}\left(l_{1}\right)$ according to

$$
\phi_{n}\left(I_{1}\right)=K^{\frac{n-1}{2}} \phi_{n}\left(l_{1}\right)=K^{\frac{n-1}{2}} \phi_{n}\left(r_{3} K^{\prime}\right)
$$

Eq. $(3.40)$ is the first of an infuite set of coupled integral equations. The uex: may be derived by contracting Eq. (3.17) on the left with two qō pairs with fractional momenta of $x_{1}$ to $x_{4}$. This equation will couple $02.0_{4}$, and $0_{6}$. Neglecting $o_{6}$. it is 


$$
\begin{aligned}
& \mu^{2}\left[N \phi_{4}(x)-\phi_{i}(\tilde{x})\right]=m^{2}\left(\sum_{i=1}^{1} \frac{1}{x_{1}}\right)\left[N \phi_{4}(x)-\phi_{4}(\dot{x})\right] \\
& -\frac{1}{2} \frac{g^{2}}{\pi}\left(\frac{N^{2}-\alpha}{2 N}\right)\left[\frac{\phi_{2}\left(x_{1}\right)-\phi_{2}\left(x_{4}\right)}{\left(1-x_{1}-x_{4}\right)^{2}}+\frac{\phi_{2}\left(x_{1}\right)-\phi_{2}\left(x_{4}\right)}{\left(1-x_{1}-x_{4}\right)^{2}}\right] \\
& +\frac{1}{2} \frac{g^{2}}{\pi}\left(\frac{1-\alpha}{2}\right)\left[\frac{\phi_{1}\left(x_{1}\right)-\phi_{2}\left(x_{2}\right)}{\left(1-x_{1}-x_{2}\right)^{2}}+\frac{\phi_{2}\left(x_{3}\right)-\phi_{2}\left(x_{4}\right)}{\left(1-x_{3}-x_{4}\right)^{2}}\right] \\
& -\frac{g^{2}}{\pi}\left(\frac{\lambda^{-2}-\alpha}{2}\right) \int\left[d_{y}\right]\left[\frac{\phi_{4}\left(y_{1}, y_{2} ; x_{3}, x_{4}\right)-\phi_{4}(z)}{\left(x_{1}-y_{1}\right)^{2}}+\frac{\phi_{4}\left(x_{1}, x_{2}: y_{3}, y_{4}\right)-\phi_{4}(x)}{\left(x_{3}-y_{3}\right)^{2}}\right] \\
& +\frac{g^{2}}{\pi}\left(\frac{\Lambda^{2}-a}{2 .}\right) \int[d y]\left[\frac{\sigma_{4}\left(y_{1}, x_{2} I_{3} \cdot y_{4}\right)}{\left(x_{1}+I_{4}\right)^{2}}+\frac{\phi_{4}\left(I_{1} \cdot y_{2} y_{3} \cdot x_{4}\right)}{\left(x_{3}+x_{3}\right)^{2}}\right. \\
& +\frac{\phi_{4}\left(y_{1}, y_{2}: x_{1}, x_{4}\right)-\phi_{4}(\dot{x})}{\left(x_{3}-y_{1}\right)^{2}}+\frac{\phi_{4}\left(x_{3}, x_{2}: y_{3}, y_{4}\right)-\phi_{4}(\dot{x})}{\left(x_{1}-y_{3}\right)^{2}} \\
& +\frac{\phi_{4}\left(y_{1}, x_{2} ; x_{3}, y_{4}\right)-\phi_{4}(\dot{x})}{\left(x_{3}-y_{1}\right)^{2}}+\frac{\phi_{4}\left(x_{3} \cdot y_{2} ; y_{3}, x_{4}\right)-\phi_{4}(\dot{x})}{\left(x_{1}-y_{3}\right)^{2}} \\
& \left.-\frac{\phi_{4}\left(x_{1}, y_{2 i} x_{3}, y_{4}\right)-\phi_{4}(\dot{x})}{\left(x_{2}-y_{4}\right)^{2}}-\frac{\phi_{4}\left(y_{1}, x_{2 i} y_{3}, x_{4}\right)-\phi_{4}(\dot{x})}{\left(x_{1}-y_{3}\right)^{2}}\right] \\
& +\frac{g^{2}}{\pi}\left(\frac{1-\alpha}{2}\right) \int[d y]\left[\frac{\phi_{4}\left(y_{1} \cdot y_{2}: x_{3} \cdot x_{4}\right)-\phi_{4}\left(y_{1} \cdot x_{4}: x_{3} \cdot y_{2}\right)}{\left(x_{1}+x_{2}\right)^{2}}\right. \\
& +\frac{\phi_{1}\left(x_{1}, x_{2}: y_{3}, y_{4}\right)-\phi_{4}\left(x_{1}, y_{2}: y_{3} \cdot x_{4}\right)}{\left(x_{3}+x_{4}\right)^{2}} \\
& -\frac{\theta_{9}\left(I_{3} \cdot I_{2}: y_{3} \cdot y_{4}\right)}{\left(x_{1}+I_{4}\right)^{2}}-\frac{\theta_{4}\left(x_{1} \cdot x_{4}: y_{3} \cdot y_{4}\right)}{\left(I_{2}+I_{3}\right)^{2}} \\
& +\frac{O_{4}\left(y_{1}, I_{2} ; y_{3}, I_{4}\right)-O_{4}\left(y_{1}, x_{2}: I_{3}, y_{4}\right)}{\left(I_{1}-y_{1}\right)^{2}} \\
& \left.+\frac{0_{4}\left(x_{1} \cdot y_{2}: x_{3} \cdot y_{4}\right)-0_{4}\left(I_{1} \cdot y_{2}: y_{3} \cdot x_{4}\right)}{\left(x_{2}-y_{2}\right)^{2}}\right] \text {, }
\end{aligned}
$$


with the wavefunctions defined such that $\phi_{2}\left(x_{1}\right) \equiv \phi_{2}\left(x_{1}, 1-x_{1}\right), \phi_{4}(x) \equiv \phi_{4}\left(x_{1}, x_{2}: x_{3}, x_{4}\right)$. $\phi_{4}(\bar{x}) \equiv \phi_{4}\left(x_{3}, x_{2} ; x_{1}, x_{4}\right)$, and $\sum x_{1}=1$. Repeating this procedure would produce equations which relate the infinite set of wavefunctions.

Having continuum integral equations which include higher-Fock wavefunctions. it is possible "s determine if in accounting for these, conclusions drawn from the valence wavefunctions are altered. From Eq. (3.40), $\phi_{4}$ could affect the small- $x$ behavior of $\phi_{2}$.
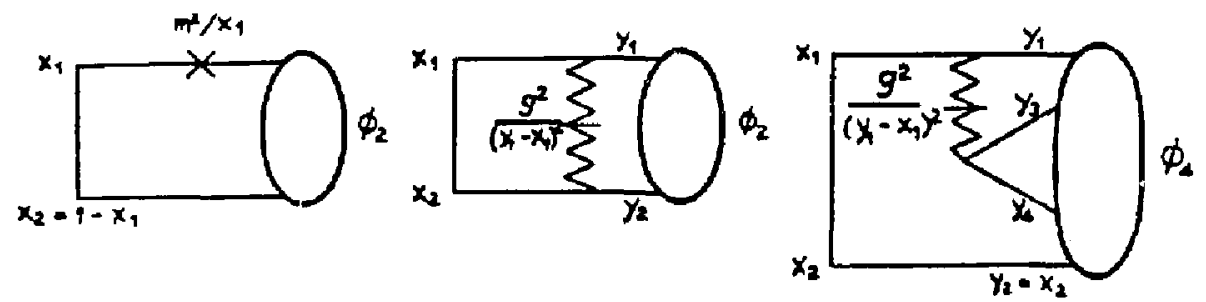

Figure 28. Typseal Diagrams which Contribute to the lntegra! Equation for $\theta_{\text {gl }}(\mathbf{r})$

That the endpoint behavior of the valence wavefunction is unmodified by in cluding higher-Fock states may be understood by tracing the source of the leading term. For $x_{1} \sim 0$, the leading kinetic term. Fig. (28a) goes as $\mathrm{m}^{2} / x_{1}$. (The modi. fication from the endpoint belavior of $\phi_{2}(x)$ may be ignored for now.) This mus: be matched by the leading term from the exchange of an instantanteous gluon. Fin. (26b). The dominant contribution from the $y$ integration comes frum the refivil where $y_{1}$ is near $x_{1}$. Taking $\Delta_{y_{1}} \sim x_{1}$, and $g^{2} / x_{1}^{2}$ from the gluon suggests a leadiag contribution whici goes as $g^{2} / x_{1}$, ma:ching the kinetic tern. The variable $g$ ifixed by momentum conservation, and so contributes noiling further. 
Higher-Fock wavefunctions will appear in interactions of which Fig. (2EC) is typical. Again, the $y_{1}$ integral is dominated by the region $y_{1} \sim x_{1}$, and with the propagator contributes $g^{2} / x_{1}$. Because $y_{2}=x_{2}=1-x_{1}$, the integration variable $y_{3}$ is restricted to be less than $x_{1}$ by $\sum y_{1}=:$, contributing another factor of $x_{1}$. This diagram will be of order zero in $x_{1}$ and as a consequence, $\phi_{4}$ does not affi it the leading behavior of $\phi_{2}$.

This may be seen explicitly in Eq. (3.40) (for $S U(N)$ ). The valence terms have already' been discussed. The $\phi_{4}$ term,

$$
-4 \frac{g_{1}^{2}}{\pi} \int_{0}^{1-x_{1}-1} d y_{4} \int_{0}^{1-x_{1}-y_{4}} d y_{3} \frac{\phi_{4}\left(x_{1}, 1-x_{1}-y_{3}-y_{4} \cdot y_{3} \cdot y_{1}\right)}{\left(1-x_{1}-y_{4}\right)^{2}}
$$

(with $\phi_{4}$ approximated by one) would contribute $+4\left(g_{N}^{2} / \pi\right) \ln \left(c /(\sigma-1) x_{1}\right)$ from the region $y \in\left[1-\sigma x_{1}, 1-x_{1}-\epsilon\right]$, while the second $\phi_{4}$ term would add $-4\left(g_{T}^{2} / \pi\right)\left(n ; \epsilon / x_{1}\right)$. The combination is finite in $\epsilon$ and order zero in $x_{1}$, as anticipated. As long as $o_{i}$ does not in reality diverge for small $I_{1}$, allowing it non-trivial behavior does not alter the conclusion.

There is nothing in this discussion which is specific 10 two and four particle wavefunctions. In general the leading endpoint behavior is determined by two-totwo quarti irteractions, regardless of the presence of spectators. This has alieady been observed for baryon valence wavefunctions, which were shown to possess the same asymptotic smail-r behavior as the valence meson.

Furthermore. this also appears to hold for higher-Fock wavefunctions. Culisitut Eq. (3.42) for $o_{4}$. As argued above. $o_{0}$ does not iresence the betavior of $c_{4}$ an $r_{1} \rightarrow 0$. Also, the monesta appearing in terms with $o_{1}$ are fixed and catant 

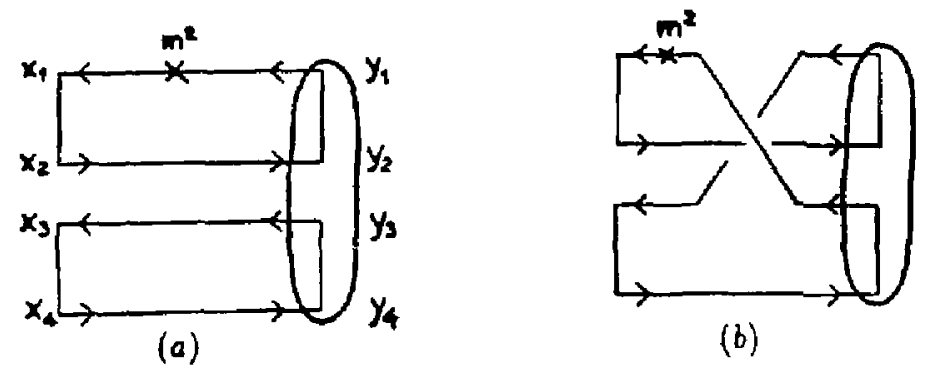

(b)

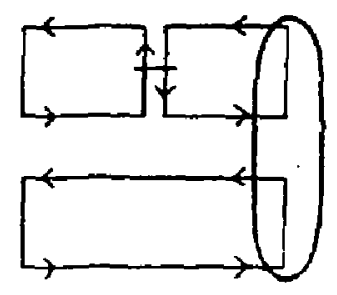

(c)
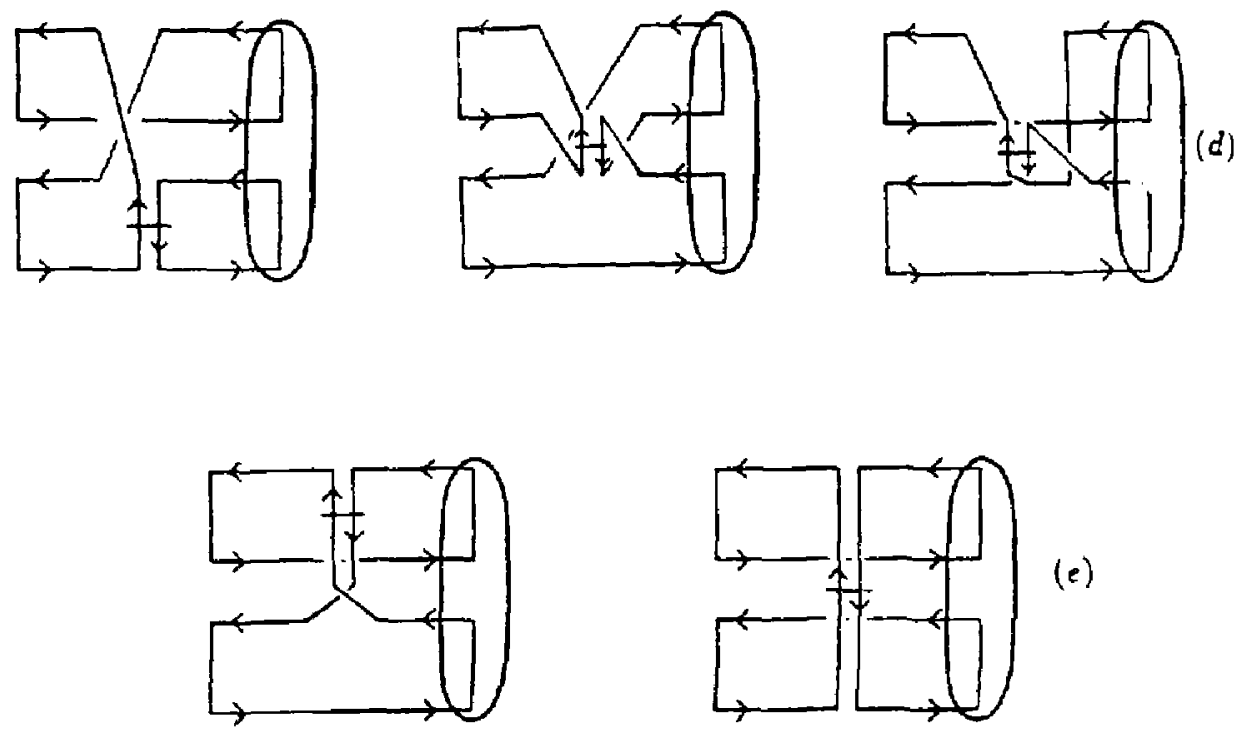

(e)

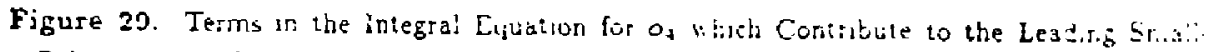

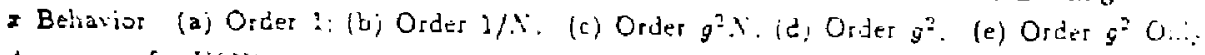
Afpeatung fo: U ( $\because$ ) 
match the kinetic term singularity of $\mathrm{m}^{2} / x_{1}$. The terms which do contribute are illustrated diagrammatically in Fig. (29). Color flow is explicitly indicated.

Only gluon exchange terms involving $x_{1}$ are relevant. Of these, two of the order $\eta^{2}$ interactions cancel, as do the $U(N)$ terms. As a result, as $x_{1} \rightarrow 0$ with the other $x_{1}$ fixed, $\phi_{4} \sim x_{1}^{a} f\left(x_{2}, x_{3}, x_{4}\right)$ with the same exponent $a$ as for the valence meson and baryon.

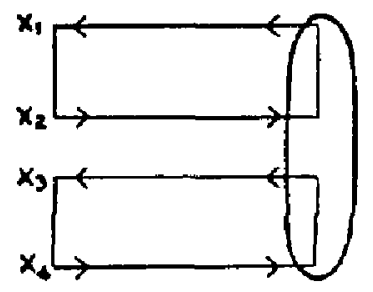

(a)
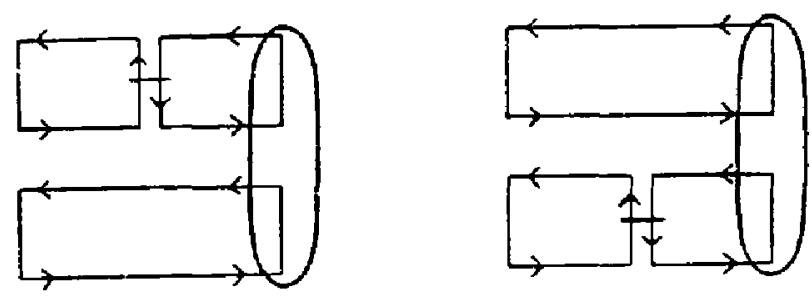

(b)

Figure 30. Leadug Terms in Large-N' Lunut for the os Integrol Equation. (a) Dlagonal Tern: Includiag Normalization, (b) Interactions

Eqs. (3.42) and (3.40) may be used to demonstrate explicitly how qư nesust decouple in the large $x$ limit. From Eq. (3.42). only the terms which correspond $i$, plarar diagrams illustrated in 5 !g (30) contribute to leading order. Note that lin pairs of color siuglets act independently to this order, even in the waveunctivi: nu: malization, and it is reasonable to expect a product of o: wavefunctions to forti: a solution for $\phi_{4}$. For example. consider the case where momentum is equa:ly sherent. $x_{1}-:=x_{3}+x_{4}=1 / 2$. Defire new nomenta $u_{1}, u_{2} \cdot l_{2} . u_{2}$ as twice $x_{1}, x_{2}, x_{2}, x_{4}$ 
respectively, so as to range from zero to one. Substituting $\delta_{2}\left(u_{1}, u_{2}\right) \phi_{2}\left(v_{1}, v_{2}\right)$ for $\phi_{4}\left(x_{1}, x_{2} ; x_{3}, x_{1}\right)$ factorizes Eq. (3.4.) to leading order in $N$ :

$$
\begin{aligned}
& M^{2} \phi_{2}(u) \phi_{2}(v)= \\
& 2\left[m^{2}\left(\frac{1}{u_{1}}+\frac{1}{u_{2}}\right) \phi_{2}(u)-\frac{g^{2} N}{2 \pi} \int_{0}^{1} d y \frac{\phi_{2}(y)-\phi_{2}(y)}{(y-u)^{2}}\right] \phi_{2}(v) \\
& +2\left[m^{2}\left(\frac{1}{v_{1}}+\frac{1}{v_{2}}\right) \phi_{2}(u)-\frac{g^{2} N}{2 \pi} \int_{0}^{1} d y \frac{\phi_{2}(y)-\phi_{2}(y)}{(y-v)^{2}}\right] \phi_{2}(v) .
\end{aligned}
$$

Evider: $1 y$ if $\phi_{2}$ is an eigenstate of the large $N N^{\prime} q \bar{q}$ equation with mass squared of $\mu^{2}$, then $M^{2}$ for $\mathrm{c}_{4}$ is just $4 \mu^{2}$; that is. the mass of two non-interacting mesons a: zero relative momentum.

Note that in Eq. (3.40). $\phi_{4}$ appears coupled to $\phi_{2}$ even to leading order in. However, for the particular case where $\phi_{4}=\phi_{2} \times \phi_{2}$, these terms cancel, and $\phi_{2}$ satisfies the large- $N$ valence Fock state equation, uncoupled to eitber higher-Fock wavefunctions or meson pairs.

Meson and baryon wavefunctions in the valence approximation exhibit a universal asymptotic behavior as a single variable approaches zero while the others remain fixed.

$$
\phi(x) \propto x_{1}^{a},
$$

with a given $\vdots:$ Eq. (3.22). This holds for all $\lambda$. Furthermure. mesonic integra! equations which incorporate the four-quark Fock state show both that its inclusion does not alter this property for the valence wavefunction, and that it shares thr same small- $x$ forn. This holds whether this four-quark state represents a pair 
of valence mesons, in which case the behavior is required for consistency, or the higher-Fock contribution to a single meson.

The interaction that provides the $g^{2} / x$ to match the kinetic $\mathrm{m}^{2} / x$ term as $x \rightarrow 0$, two-quark to two-guark instantaneous gluon exchange, preserves quark number. Because this interaction appears in essentially the same way in all integral equations, it is likely that Eq. (3.22) holds for all wavefunctions, whether valence or higher-Fock, and that this behavior is unaffected by interaction with states of bigher or lower quark number.

Numerical errors are due to both the endpoint behavior and the priticipal value prescription for the gluon propagator, as well as those usually associated with numerical integration. The principal value prescription provides the same ordet I/ $\mathrm{h}$ error in all cases. If the endpoint wavefunction behavior is also unit ersal. then for a given $\mathrm{g} / \mathrm{m}$, the errors for all quantities computed will bchave in the same manner as that desrribed for the valence meson, Eq. (3.39). In any case. it has been shown to be true generally enough to make Richardsull extrapolatiol: applicable in most cases of interest. In particular, the lightest meson and baryon are dominated by their valence wavefunctions up to fairly strong coupling. and it is certainly applicable there.

\subsection{HEAVY-QLARI LIMIT FOR NESONS}

In order to understand the large quark mass limit of low-Jying meson massm and wavefunctions, the large mass limit of the meson integral equation

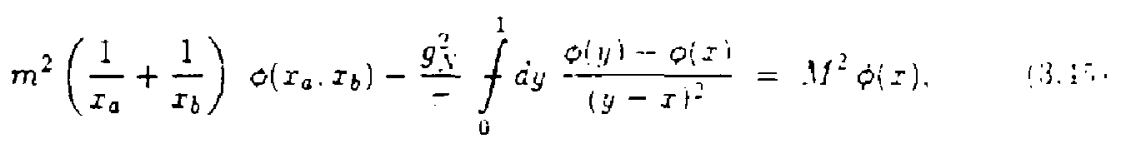


with

$$
g_{N}^{2} \equiv g^{2}\left(\frac{N^{2}-1}{2 N}\right) \quad \text { and } \quad x_{a}+x_{b}=1
$$

may be taken directly. In this limit, higher-Fock components may be neglected, as they contain a larger number of the very massive quarks. Were there physical. propagating gluons, higher states containing arbitrary numbers of gluons in addition to the heavy quark pair would either be included explicitly or incorporated into an effective $q \bar{q}$ potential. Unlike the formulation in the infinite monentum frame, no special frame has been selected, and a nonrelativistic limit of Eq. (3.45) is perfectly sensible.

Furthermore, such a reduction should help to clarify the connection between wavefunctions in nonrelativistic quantum mechanics and those on the light cone. Following Ref. [21], the expansion may be performed by counting powers of quark velocity (small) and mass (large). Let $k_{a}$ and $k_{b}$ be the equal-time nomentum in the $I^{3}$ direction. of order $m i$. Then

$$
\begin{aligned}
k_{a}^{ \pm} & =\left(m^{2}+k_{a}^{2}\right)^{1 / 2} \pm k_{a} \\
& \sim m \pm k_{a}+\frac{k_{a}^{2}}{2 m}+\cdots,
\end{aligned}
$$

with terms of order $m, m v$ and $m v^{2}$, respectively: After dividing Eq. (3.4.j) :hrough by the total light-cone momentum $P^{+}$to convert $r_{a}$ and $I_{b}$ to $h_{a}^{+}$and $h_{b}^{-}$fiom $k^{+}=I P^{+}$, then. in terms of the equal-time momentum sim and difierence.

$$
k \equiv k_{0}+k_{b} \quad \text { and } \quad q \equiv k_{0}-k_{b}
$$


the expanded kinetic term is

$$
m^{2}\left(\frac{1}{k_{a}^{+}}+\frac{1}{k_{b}^{+}}\right) \sim 2 m-k+\frac{1}{4 m}\left(k^{2}+q^{2}\right)+\ldots
$$

Defining the binding energ: $E$ by

$$
E \equiv M-2 m i=\mathcal{O}\left(m v^{2}\right)
$$

the right-hand side of Eq. (3.45) reduces to

$$
\begin{aligned}
\frac{M^{2}}{P^{+}} \phi(x) & =\frac{(2 m+E)^{2}}{k_{a}^{+}+k_{b}^{+}} \phi(x) \\
& \sim\left(2 m-K^{\prime}+2 E+\frac{\left(k^{2}-q^{2}\right)}{4 m}+\ldots\right) \phi(x)
\end{aligned}
$$

Note that the arguments of $\phi(x)$ in this limit, $x_{a}$ and $x_{b}$, become

$$
\begin{aligned}
& x_{a} \sim \frac{1}{2}+\frac{q}{4 m}+\ldots . \\
& x_{b} \sim \frac{1}{2}-\frac{q}{4 m}+\ldots .
\end{aligned}
$$

As discussed in Ref. [24], light-cone wavefunctions involving $n$ heavy quarks wi]] be strongly peaked around $x \sim 1 / n$, or $1 / 2$ in this case. The nonselativistic wave unction is then

$$
o(q) \equiv o\left(\frac{1}{2}+\frac{q}{4 m} \cdot \frac{1}{2}-\frac{q}{4 m}\right)
$$

Finaly: the potential term becones. afier replacing $y$ with $1 / 1=+\varphi^{\prime} / 4: \cdots$ ant 
letting $p=q^{\prime}-q$,

$$
-\frac{4 g_{N}^{2} m}{\pi P^{+}} \int_{-2 m}^{2 m} \frac{d p}{p^{2}}[\phi(p+q)-\phi(q)]
$$

Keeping the leading term from $P^{+}$, and assuming that the wavefunctions fall off quickly enough to extend the integration to infinity, Eq. (3.45) is finally

$$
\frac{q^{2}}{4 m} \phi(q)-\frac{g_{N}^{2}}{\pi} \int_{-\infty}^{\infty} \frac{d p}{p^{2}}[\phi(p+q)-\phi(q)]=E \phi(q) .
$$

In terms of the position-space wavefunction

$$
\phi(z)=\frac{1}{(2 \pi)^{1 / 2}} \int_{-\infty}^{\infty} d q e^{-1 q z} \phi(q) .
$$

the interaction term transforms into the loca! potential

$$
Y(z) \equiv \int_{-\infty}^{\infty} \frac{d p}{p^{2}}\left[e^{-i p z}-1\right]=-\pi \mid z 1
$$

Note that this principal value prescription does not generate an additional finite renormalization of the quark mass. Eq. (3.54) becomes the Schrodinger equation

$$
\left[-\frac{1}{4 n} \partial_{z}^{2}+g_{N}^{2}|z|\right] \phi(z)=E O^{\prime}(z)
$$

This derivation required the relevant momenta in the wavefunction to be much less than twice the quark mass; for example, it was used to obtain Eq. (3.5i) from Eq. (3.53). in other words, the position-space wavefunction is sensible as long at distances as small as the Compton wavelength of the quarks are not probed. Where. for example, pair production would foil a probabilistic interpretation of oiz! 
This equation and its solutions are discussed in Ref. [37]. Introducing dimensionless variables

$$
u \equiv\left(4 g_{N}^{2} m\right)^{1 / 3} z \quad \text { and } \quad \lambda \equiv\left(\frac{4 m}{g_{N}^{4}}\right)^{1 / 3} E
$$

the equation becomes

$$
\left[-\partial_{u}^{2}+|u|\right] \phi(u)=\lambda \phi(u)
$$

The solutions are Airy functions,

$$
\varphi_{n}(u)=A_{1}\left(u-\lambda_{n}\right) .
$$

with eigenvalues given by

$$
A_{1}^{\prime}\left(-\lambda_{n}\right)=0
$$

for symmetric (parity' odd) states, and

$$
\left.A_{2} !-\lambda_{n}\right)=0
$$

when antisymmetric (parity evell).

The lowest meson mass

$$
\frac{M_{1}}{g}=\frac{2 m}{g}+\left(\frac{g}{4 m}\right)^{1 / 3}\left(\frac{x^{2}-1}{2 A^{2}}\right)^{2 / 3} \lambda_{1}
$$

is plotted in Fig. [31] for $N=2.3$ and 4 and compared with the ectua! output. Note that in all cases, the binding energy $E$ is positive. The actual dat: as: $m_{i}$. totica!ly approaches the nonrelativistic limit from below. reaching to within a':s:s: 57 for $m / g=1.6$. 


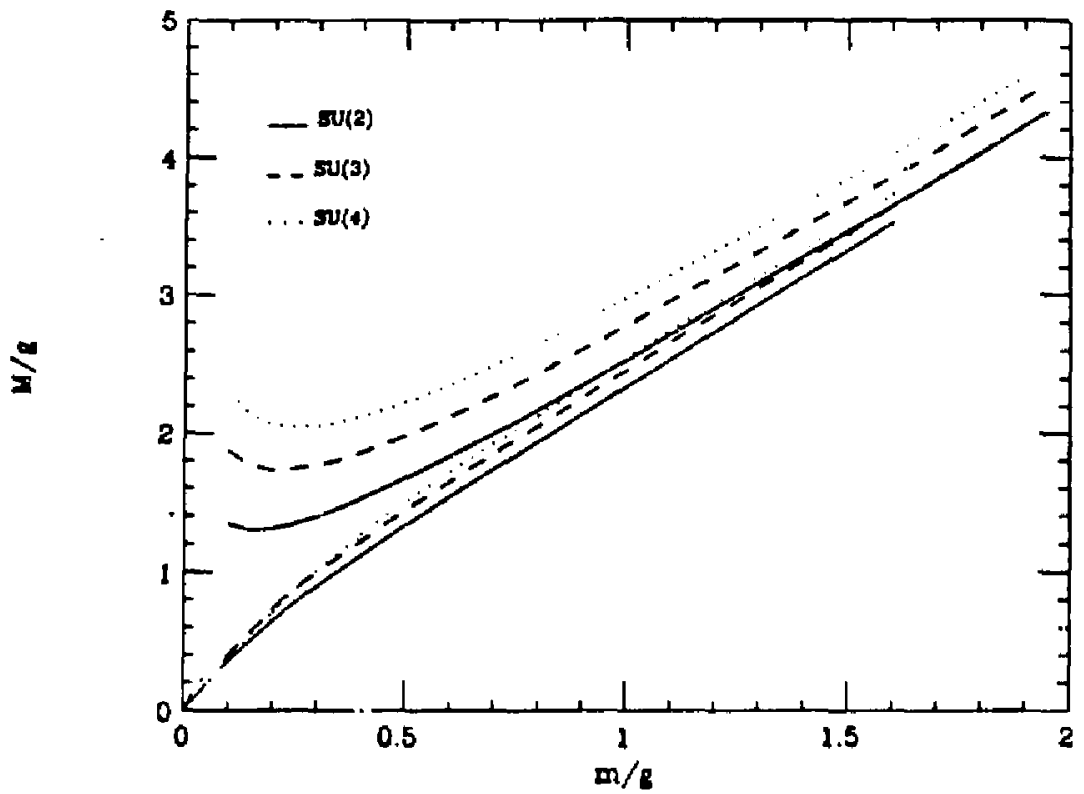

Figure 31. Comparison of Lightest Meson Mass for $N=2,3$, and 4 with Nonrelativistic Solutions.

\subsection{HEAVY-QUARI LIMIT FOR BARYONS}

The same nonrelativistic reduction may be applied to the minimum Fock state equation for the baryon. Three colors will be considered here, although the extension of the nonrelativistic Schrodinger equation to baryons of larger $\Lambda$ will be evident. As a consequence, the confining potential for three (or more) very massive quarks may be derived from first principles.

The light-cone eigenvalue equation for the $\Lambda^{\prime}=3$ baryon restricted to three quarks is 


$$
\begin{aligned}
& m^{2}\left(\frac{1}{x_{a}}+\frac{1}{x_{b}}+\frac{1}{x_{c}}\right) \phi(x)-\frac{g^{2}}{\pi}\left(\frac{N+1}{2 N}\right)[ \\
& \quad \int_{a}^{x_{a}} \frac{d s}{s^{2}}\left[\phi\left(x_{a}-s, x_{b}, x_{c}+s\right)+\phi\left(x_{a}-s, x_{b}+s, x_{c}\right)-(\Lambda-1) \phi(x)\right] \\
& +\int_{c}^{x_{b}} \frac{d s}{s^{2}}\left[\phi\left(x_{a}+s, x_{b}-s, x_{c}\right)+\phi\left(x_{a}, x_{b}-s, x_{c}+s\right)-(N-1) \phi(x)\right] \\
& \left.+\int_{c}^{x_{c}} \frac{d s}{s^{2}}\left[\phi\left(x_{a}, x_{b}+s, x_{c}-s\right)+\phi\left(x_{a}+s, x_{b}, x_{c}-s\right)-(\lambda-1) \sigma(x)\right]\right] \\
& =M^{2} \phi(x) .
\end{aligned}
$$

where $I_{a}+I_{b}+I_{c}=1$. Proceeding as for the meson, the equation is divided through by $P^{+}$, the $k_{t}^{+}$are expanded in powers of velocity over mass, and the binding energy is defined to be

$$
E \equiv M-3 m
$$

In ternis of the relative monenta

$$
\begin{gathered}
K=k_{a}+k_{b}+k_{c} . \\
p=k_{a}-k_{b} . \\
q=k_{b}-k_{c} . \\
p+q=k_{a} \rightarrow k_{c} .
\end{gathered}
$$

where the $k_{1}$ are the equal-tume spatial momenta. Eq. (3.64) reduces to

$$
\frac{1}{3 m}\left(p^{2}+q^{2}+(p+q)^{2}\right] \phi(x)+\operatorname{Vo}(r)=2 E o(r)
$$


with the potential I' still to be computed. As before, the total momentun $K$ has dropped out.

The arguments of the wavefunction, $x_{i}$, to leading nontrivial order in $p$ and $q$, become

$$
\begin{aligned}
& x_{a} \sim \frac{1}{3}+\frac{2 p+q}{3 m}, \\
& x_{b} \sim \frac{1}{3}+\frac{q-p}{3 m}, \\
& x_{c} \sim \frac{1}{3}-\frac{(2 q+p)}{3 m} .
\end{aligned}
$$

The nonrelativistic momentum-space wavefunction is then determined from the light-cone wavefunction by

$$
\begin{aligned}
\phi\left(x_{a}, x_{b}, x_{c}\right) & \sim \phi\left(\frac{1}{3}+\frac{2 p+q}{3 m}, \frac{1}{3}+\frac{q-p}{3 m}, \frac{1}{3}-\frac{(2 q+p)}{3 m}\right) \\
& \equiv \phi(p, q) .
\end{aligned}
$$

To compute the potential $V$, consider the first potential term of Eq. (3.6t). Expanding the arguments of $o(x)$ as in Eq. (3.69). and defining a new integraiton variable $t=m s$. the leading contribution from this iniegral is

$$
m \int_{0}^{m / 3} \frac{d t}{t^{2}} o(p-t, q-t) .
$$

Including the term where $s$ appears with the opposite sign. as we:" as the terms necessary to keep the integral well-defined gives an interaction of the form

$$
m \int_{-m / 3}^{m / 3} \frac{d t}{t^{2}}\left(o(p-t . q-t)-o(p . q)^{\prime} .\right.
$$

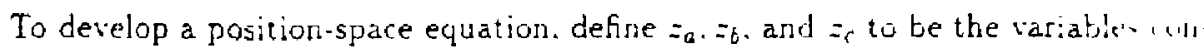


jugate to $k^{a}, k^{b}$, and $k^{c}$. Then the variables conjugate to $S, p$, and $q$, respectively: are

$$
\begin{gathered}
\bar{z}=\frac{1}{3}\left(z_{a}+z_{b}+z_{c}\right) . \\
z_{p}=\frac{1}{3}\left(2 z_{a}-z_{b}-z_{c}\right) . \\
z_{q}=\frac{1}{3}\left(z_{a}+z_{b}-2 z_{c}\right) .
\end{gathered}
$$

Defining the posstion-space wavefunction by

$$
\phi(p . q)=\int_{-\infty}^{\infty} \frac{d z_{p} d z_{q}}{(2 \pi)} e^{-1\left(p z_{r}+q z_{q}\right)} \phi\left(z_{p} . z_{q}\right)
$$

and converting Eq. (3.i1) into a position-space potential, the Schrodinger equation for $\phi(z)$ is

$$
\begin{aligned}
& {\left[-\frac{1}{6 m^{2}}\left(\partial_{x_{p}}^{2}+\partial_{z_{q}}^{2}+\left(\partial_{z_{p}}+\partial_{x_{q}}\right)^{2}\right)\right.} \\
& \left.+\frac{g^{2}}{6}\left(\frac{N^{\prime}+1}{2 N}\right)\left(\left|z_{a}-z_{b}\right|+\left|z_{a}-z_{q}\right|+\left|z_{b}-z_{c}\right|\right)\right] \partial(z) \\
& =E \phi(z) .
\end{aligned}
$$

The variables $z_{a}, z_{b}$, and $z_{c}$ are understood to be functions of $z_{i}$ and $z_{y}$. The extension of this equation to $\Lambda^{\prime}$ other than three is now straightforward.

To sketch the method for solving Eq. (3.74), tewrite it in terms of the variabies

$$
r=\frac{1}{3}\left(z p+z_{q}\right) \quad \text { and } \quad t=z_{r}-z_{q}
$$


These diagonalize the derivatives, and Eq. (3.74) is now

$$
\begin{aligned}
& {\left[-\frac{1}{3 m}\left(\frac{1}{9} \partial_{r}^{2}+\partial_{i}^{2}\right)+\frac{g^{2}}{4}\left(\frac{N+1}{2 N}\right)(|r+t|+2|r|+|r-t|)\right] \phi(r, t)} \\
& =E \phi(r, t)
\end{aligned}
$$

This may be solved by separation of variables

$$
\phi(r, t)=R(r) T(t) .
$$

yielding the equations

$$
\begin{aligned}
& -\frac{1}{2 \pi m} R^{\prime \prime}+\alpha \frac{g^{2}}{2}\left(\frac{\Lambda+1}{2 \Lambda}\right) r R=\lambda R \\
& -\frac{1}{3 m} T^{\prime \prime}+\beta \frac{g^{2}}{2}\left(\frac{\Lambda+1}{2 \Lambda}\right) t T=(E-\lambda) T .
\end{aligned}
$$

where $\beta=0, \pm 1$ and $a= \pm 1, \pm 2$ depending on the region of $r$ and $t$. The solutions are again Airy functions, except when $\beta=0$, and eigentalue conditions may be derived by matching solutions at the varjous boundaries of $r$ and $t$.

\subsection{MASSLESS MESONS AND BARYONS AT STRONG COLPLING}

As discussed previously: the momentum spare transforms of the $S[1 \mathrm{~N}$ : currents $\left(\right.$ at $\left.x^{+}=0\right)$

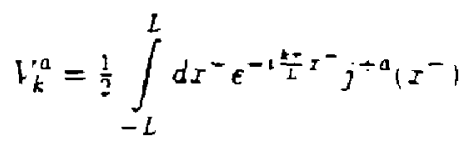

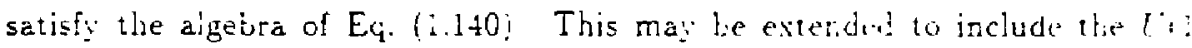


curredt

$$
j^{+(0)}=2: \psi_{R}^{1} T^{0} \dot{L}_{R}:
$$

with $T^{0}=\frac{1}{\sqrt{2}} 1$. The transformed operator $v_{k}^{0}$ commutes with the other $S(1)$ elements, and satisfies

$$
\left[T_{k}^{0}, V_{\ell}^{0}\right]=\frac{1}{2} \ell \varepsilon_{k+\ell, 0}
$$

Noting that $l_{k}^{\cdot a \dagger}=V_{-k}^{a}$, this may be rewritten

$$
\left[l_{t}^{\prime 0 \dagger}, l_{\ell}^{0}\right]=\frac{1}{2} \ell \delta_{k, l}
$$

The interacting part of the Hamiltonian is greatly simplified when expressed in terms of these operators:

$$
P_{I}^{--}=-\frac{g^{2}}{16} \int_{-L}^{L} d x^{-} d y^{-} j^{+a}\left(x^{-}\right)\left|x^{-}-y^{-}\right| j^{+a}\left|i^{-}\right|
$$

becomes

$$
\frac{L}{\pi} \frac{g^{2}}{4 \pi} \sum_{k=-\infty}^{\infty} \frac{1}{k^{2}} V_{k}^{a} V_{-k}^{a} .
$$

Because $l_{0}^{a}=Q^{a}$, the contribution at $k=0$ is proportional to i be toid rib: ge $Q^{a} Q^{d}$ and so may be discarded.

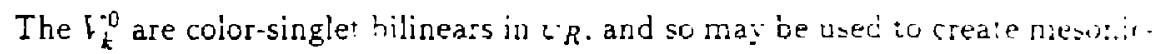
like states. Specificaly. the related operators (for $k>0$ by definition!

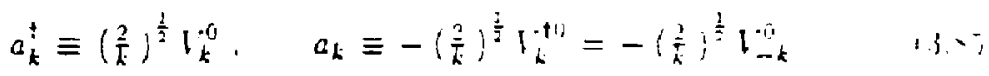


satisfy standard free boson commutation relations

$$
\left[a_{k}, a_{\ell}^{\dagger}\right\rfloor=\delta_{k, \ell}
$$

and so $V_{k}^{0}$ creates a boson with momentum $P^{+}=2 \pi k / L$. In the limit where $m / g$ is zeto, the entire Hamiltonian is given by Eq. (3.81). Because the $V_{k}^{0}$ commute with the $V_{k}^{a}$ which appear in $P_{I}^{-}$,

$$
M f^{2} V_{k}^{0}|0\rangle=\frac{2 \pi \dot{k}}{L}\left[P^{-}, V_{k}^{0}\right]|0\rangle=0 \text {. }
$$

Not only is the state created by acting with $l_{k}^{r 0}$ on the vacuum an exactly massid:eigenstaie in this limit, but states formed by repeated applications are also exictiy massless. Furthermore, acting with $V_{k}^{0}$ on an eigenstate of non-zero mass produces a degenerate state of opposite parity, as will be demonstrated. This argunent in independent of the value of the numerical momeztum $H$ and so gives an exaci continuum result. [3] $^{\text {s. }}$

The firiteness of $K$ becomes relevant. however, when couning the ram!ne: v: massless mesons which can be produced. Massiess mesoric states of to:ia'. nuna:er. ical momentum $h$ of 10/2. for example. may be constructed by from one to five applications of $i_{k}^{r 0}$ on the racuum, with momentum distributed as $(5),(1,4),(2,3)$. (1.1.5; (1,1.2) (1.1,1,2) and (1.1,1,1.1). Comparing wilt Fig. (4a) 1:este ar. count for all seven of the states whose masses vanish in thio limit. Increasitig $K$ permits an increasing number of massless states. Whicl: brcones infritir in il." continum limit.

Just as the existence and number of massless states is must simply discu.n... in terms of the $l_{j}^{u}$, so also are the wavefunirtions of thes siales. Applying on $l_{i}$ 
to the vacuun.

$$
V_{K}^{0}|0\rangle=\frac{1}{\sqrt{2 N}} \sum_{n=\frac{1}{2} \cdot \frac{3}{2} \ldots}^{H-\frac{1}{2}} b_{K-n}^{\dagger c} d_{n, c}^{\dagger}|0\rangle
$$

yields a continum wavefunction of $\phi(I)=1$, in the continuum, $n / \lambda$ becomks $I$. Because $\phi$ is even under the interchange of $x$ and $1-x_{1}$ this state is a pseudoscalar. The wavefunctions of momentum $K$ created by applying $V^{\prime 0}$ twice are, for any $\varepsilon<i$

$$
\begin{aligned}
& v_{h-\ell}^{0} l \cdot 0|0\rangle=\frac{1}{2 . \top}\left[\sum_{n=\frac{1}{2}}^{\ell-\frac{1}{2}}\left(b_{h-n}^{\dagger c} d_{n, c}^{\dagger}-b_{n}^{\dagger c} d_{h-\pi, c}^{\dagger}\right)+\right. \\
& \left.\sum_{m=\frac{1}{2}}^{h-\ell-\frac{1}{2}} \sum_{n=\frac{1}{2}}^{\ell-\frac{1}{2}} b_{h-c+m}^{\dagger c_{2}} d_{m, c_{2}}^{\dagger} b_{t-n}^{\dagger \varepsilon_{1}} d_{n, c_{1}}^{\dagger}\right]|0\rangle .
\end{aligned}
$$

The $q \bar{q}$ piece is odd and so this state is a scalar, as a product of pseudoscalars nast be. Al! the massless meson wavefunctions for a given $K$ may be construcied in this manner, and parity will alternate with each additiona! $f^{-\dot{v}}$.

Two further comments should be made. First, each additional $t^{\circ}$ increases the particle number of the highest Fock state, which has a coefficient of order one. by one $g \bar{q}$ pair. Pecause these states are degenerate as $\mathrm{m} / \mathrm{g} \rightarrow 0$. it is possible that a great deal of mixing occurs. and Foch states with ary number of quanta migh: be expected to appear with arbitrary strength in any wavefunction. In fact, for finite but small $\pi / g$, the lower states are approximaiely ordered accordirg to it.t number of $\mathrm{F}^{\circ} \mathrm{s}$. The first state is almost entirely composed of $V_{K}^{0}|0\rangle$, the seculid by $\left.\right|_{h / 2} ^{0} l_{h / 2}^{0} 10 \%$ and so on. There is however a smal: but persistent fourgian 
component for the lightest meson which is given in Table [5]. This compontent drops rapidly with $\Lambda$, decreasing by a factor of ten from $N=2$ to 3 , and again to $N=4$, as in Fig. (19a).

Second, these states are massless and described in terms of the bosonic operators $V_{k}^{0}$ cnly when $\mathrm{m} / \mathrm{g}$ is identically zero. For small but finite $\mathrm{m} / \mathrm{g}$, the free Hamiltonian contains terms like $m^{2} / x_{i}$, and so is singular when constituent $i$ carries zero momentum. Wavefunctions must therefore vanish a: these endpoints, whereas those described above for $n / g$ identically zero do not; $m / g \rightarrow 0$ is evident? a sin. gular point in this formalism and perbaps in the theory! . Although it need no: have been the case, it will be shown that $\mathrm{m} / \mathrm{g}$ finite but approaching zero. the solutions for $\mathrm{m} / \mathrm{g}=0$ provide an accurate description of both the spectrum and the wavefunctions, apart from small regions around $x_{1}=0$, where the wavefunctions must vanish.

If the gauge group were $U\left(\Lambda^{\prime}\right)$ rather than $S C(N)^{i 00}$ the additional term associated with the extra $[(1)$

$$
\frac{L}{\pi} \frac{g^{2}}{4 \pi} \sum_{k=-\infty}^{\infty} \frac{1}{k^{2}} V_{k}^{0} v_{-k}^{0}=\frac{L}{\pi} \frac{g^{2}}{4 \pi} \sum_{k=1}^{\infty} \frac{1}{k} a_{k}^{\dagger} a_{k}+\text { constant }
$$

appears in $P^{-}$. The $a_{k}$ satisfy free bosonic commutation relations, and this addjtional interaction is therefore the discrete light-cove Hamiltonian for free bosons of mass squared $g^{2} / 2 \pi$. These formerly massless states created by the $a_{k}^{\dagger}$ are pru moted to the free massive bosons found in the Schwinger model and discussed Rers. [23? and [20]. The wavefunctions for these states however are unchanged.

Note that while the entire $(1)$ spectrum may be built up from these nuninteracting bosuns. for $[(A)$ or $S l(A)$ they desrribe on! part of the spectrun. 
these are the massless mesons for $S U(N)$. In addition there are massive states composed predominantly of excited $q \tilde{q}$ pairs.

The $L^{\prime}(1)$ (Schwinger) model has been solved exactly by establishing a correspondence between the current $j^{\mu(0)}$ and a scalar field ${ }^{(4)}$

$$
j^{\mu(0)}(x) \equiv \frac{1}{\sqrt{2 \pi}} \epsilon^{\mu \nu} \partial_{\nu} \phi^{0}(x)
$$

By means of the axial anomaly, this field may be shown to satisfy the equation of motion for a free massive scalar field, with a mass squared of $g^{2} / 2 \pi$. Not surprisingly, the operators $a_{k}^{\dagger}$ and $a_{k}$ discussed above are the creation and annihi. lation operators which result from quantizing $\phi^{0}(x)$ on the light cone with periodic boundary conditions in $x^{-}$. The scalar $\phi^{0}(x)$ expanded in these operators,

$$
\phi^{0}\left(x^{-}\right)=\frac{i}{\sqrt{2 \pi}} \sum_{\ell>0}\left(\frac{1}{2 l}\right)^{\frac{l}{2}}\left[a_{\ell} e^{-i \frac{i \pi x^{-}}{L}+a_{\ell}^{\dagger} e^{l i \pi} x^{-}}\right]
$$

satisfies the standard light-cone commutation relation

$$
\left[\varphi^{0}(x), \partial_{-} \varphi^{0}(y)\right]_{z^{+}=y^{+}=0}=-\frac{i}{2} \delta\left(x^{-}-y^{-}\right)
$$

For a discussion of bosonization for the non-Abelian currents, see Ref. $142^{\circ}$

A similar argumect may be advanced to show that in this linit there are mas:less baryons as well. The commutator of the $Y_{k}^{a}$ which appear in the interacting part of the Hamihonian. and $N$ products of the field $L_{L}^{i}(x)$ at the paint $\left(0 . x^{-}\right)$is

$$
\begin{aligned}
& {\left[l_{k}^{a}, \psi_{R}^{t c_{2}}\left(x^{-}\right) \cdots \psi_{R}^{t+c_{N}}\left(x^{-}\right)\right]=}
\end{aligned}
$$

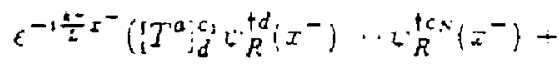

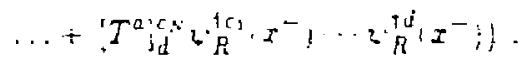


The fields multiplied at the same point are all adjoints, and singularities which are regulated by point splitting should be absent.

The entire $k$ dependence appears in the overall factor $\epsilon^{-i \frac{k \pi}{L} x^{-}}$. Consequently: noting that $V_{k}^{a}$ at $k=0$ is the charge operator $Q^{a}$.

$$
\left[V_{k}^{a}, \psi_{R}^{\dagger c_{1}} \cdots \psi_{R}^{\dagger c_{N}}\right]=e^{-i \frac{k+}{L} x^{-}}\left[Q^{a}, \psi_{R}^{\dagger c_{1}} \cdots \psi_{R}^{\dagger c_{N}}\right]
$$

If the fields are contracted with $\epsilon_{c_{3} \cdots c_{N}}$ to form an $S t^{\prime}\left(\Lambda^{*}\right)$ singlet, this commulator will vanish.

In particular, the composite baryon field

$$
B_{k} \equiv \frac{1}{2} \int_{-L}^{L} d x^{-} e^{-i \frac{k \pi}{L} x^{-}} c_{c_{i} \cdots c_{N}} \psi_{R}^{\dagger c_{1}}\left(x^{-}\right) \cdots \psi_{R}^{+c_{N N}}\left(x^{-}\right)
$$

commutes with the $V_{k}^{a}$, and, in the iimit $m / g \rightarrow 0$, with the Hamiltonian $P^{-}$. As in the case for mesons, this field creates an identically massless baryon. Repeated applications on the vacuum produce a massless state with arbitrarily desired baryon number. Furthermore, degenerate states with the same baryon number may be created by acting with the massless mesonic operators $l_{k}^{0}$ in conjunction with $B_{k}$. Again, these results are independent of $s$ and are true in the continuun: limit.

The (unnormalized) wavefunction associated with this massless baryon is

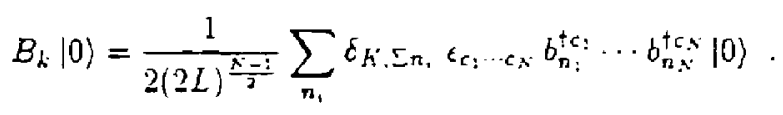

Whether this state is a fermion or boson dejends on . I beirg add or even. 
As an illustration, the normalized state for $N=3$ obtained from $B_{k}|0\rangle$ is

$$
\frac{\varepsilon_{c_{1} c_{3} c_{3}}}{\left[18\left(K+\frac{1}{2}\right)\left\{K^{\prime}-\frac{1}{2}\right)\right]^{\frac{1}{2}}} \sum_{n_{1}, n_{2}} b_{n_{1}}^{t \epsilon_{1}} b_{n_{2}}^{t \epsilon_{2}} b_{K^{\prime}-n_{1}-n_{2}}^{t c_{3}}|0\rangle .
$$

The quarh structure function derived from this wavefunction,

$$
q\left(x_{\ell}\right) \equiv K\left\langle B\left|b_{\ell}^{\dagger c} b_{\ell, c}\right| B\right\rangle=\frac{6\left(1-\ell / K^{\prime}\right)}{\left(1+1 / 2 K^{\prime}\right)\left(1+1 / 2 K^{\prime}\right)}
$$

becomes $6(1-x)$ in the continuum limit. This is the $x$ dependence clearly evident in Fig. (10). Note ibat this satisfies the baryon number sum rule

$$
\int_{0}^{1} d x 6(1-x)=3
$$

The general expression for the guark structure function for a single baryon in the $\mathrm{m} / \mathrm{g} \rightarrow 0$ limit is

$$
q(x)=N(N-1)(1-x)^{N-2} .
$$

For $\Lambda=2, q(x)=2$, which apart from the normalization, is idertical to the meson structure function for all $N$.

\subsection{EXCITED MESON STATES AT STRONG COL:PLING}

In the limit of vanishing quark mass. the presence of massless decuupled mesors allows the lightest sector of the meson spectrum to be interpreted simply. but it enormously complicates the excited states. For weakly interacting states. the seperation of the spectrum into states with several mesons, as opposed to single excitu! $g \bar{q}$ states was obvious. Excited meson wavefunctions are composed predominant? 
of two quarks, while meson-pair wavefunctions, by contrast, are products of two single meson wavefunctions which are also individually present in the spectrum. Meson-pair wavefunctions contain predominantly two quark and antiquark pairs. and their masses begin at the sum of the single meson masses and increase with relative velocity, and so are easy to discriminate.

The situation is quite different as the quark, (and meson) mass vanishes. In two dimensions. two massless particles may be combined while leaving the total invariant mass zero, even when they possess a zonzero relative mornentum. One consequence discussed earlier was the large number of massless states, limited only by the ability to subdivide a finite momentum $K^{\prime}$, created by repeatedly acting on the vacuum with the composite pseudoscalar field $V_{k}^{0}$ wbich commutes with the Hamiltonian.

Furthermore. excited, finitr-mass states will occur in clusters of nearly equal mass and alternating parity as the quark mass vanishes. The same pseudoscalar fields which create the cluster of massless mesons can also be added to excited $q \bar{q}$ states. As $k$ vanishes, their masses become degenerate. It is, in genesal, difficult and perhaps not meaningful to try to distinguish an excited quark-antiquark state from one with additional massless mesons, or from pairs of mesons. As the quark mass diminishes there is, in general. a large degree of mixing. and Fock states with all number of quarks give non-negligible contributions to alnost all warefunctions.

It is possible, however, to present a fairly simple picture of the total spectrun: in this limit, even while difficult to characterize individual states by their watcfunctions. Consider first the spectum of states when the Fock space is resiricted 
to a $q \bar{q}$ pair. The wavefunctions for the first four states are pictured in Fig. (9e) for very strong coupling or small mass, $m / g=.1$.

These states $\phi_{n}(x)$ are clearly' well described, except at the endpoints, by

$$
\phi_{\mathbf{n}}(x) \sim \cos n \pi x
$$

An argument from Refs. [36] and [30] which applies to excited states at moderate couplings may be adapted to explain why a wavefunction of the form in Eq. (3.102) might be expected. The ...iable $x$ in the $q \bar{q}$ equation for $\varphi(x)$.

$$
\frac{m^{2}}{x(1-x)} \phi(x)-\frac{g_{N}^{2}}{\pi} f_{d}^{1} \frac{\phi(y)-\phi(x)}{(y-x)^{2}}=M^{2} \phi(x) \text {. }
$$

may be reinterpreted as a position variable restricted to $x \in[0,1]$, ar. $\rfloor \mathrm{m}^{2} /[\mathrm{r}(1-$ $x)$ ] as a confining potential. The convalution integral would be suughy equitalert to $\left.g_{N}^{\mathbf{2}} \mid \hat{\boldsymbol{p}}\right\} \phi(x)$ if $y$ were integrated to infinity, with $\hat{\boldsymbol{p}}$ acting as the momentum operator conjugate to $x$. 't Hooft and Coleman argue that for high!y excited "tate" the (pseudo)potential $\mathrm{m}^{2} / x(1-x)$ could be ignored, apart from requiring : ia $\phi(x)$ vanish at the endpoiats. The ejgenstates are then

$$
\phi_{n}(x) \sim \sin n \pi x
$$

with

$$
M^{2} \approx g_{i}^{2}-n
$$

However. for the sirong coupling case aljove. while of $x$ ) must vanish at the ex:points for any finite $m$, to understand the wavefunctions and eigenvalues, it is $a_{j}$, parently more appropriate to ignore the extrene endprint behavior and to re..:? 
instead that $\phi^{\prime}(x)$ vanish there. To see why this is the case, consider computing $M^{2}$ by taking the expectation value of the Hamiltonian in an eigenstate $\phi(x)$. The kinetic term endpoint singularities will be shown to cancel one power of $m$, and $M^{2}$ for a massless meson will go to zero as approximately a single power of $m$. However, since this kinetic contribution still vanishes with $m$, both the wavefunc tion, apart from the very ends, and the mass $M$ for massive states are governed by the interaction term proportional to $g^{2}$. The endpoint contributions to this integral are potentially singular. It is en zgetically favorable, then, for $\phi(x)$ to go to a constant in this region to cancel one power of the denominator; that is. for $\phi^{\prime}(x)$ to vanish. Eigenstates are then given by cosines rather than sines.

An estimate of $M^{2}$ based on this picture may be obtained by computing the expectation value numerically, using $\varphi(x)=\sqrt{2} \cos n \pi x$. The estimates for the first few states, given in Table $[9$. describe the spectrum to within about $10 \%$.

Table [9]

\begin{tabular}{|cc|}
\hline$n$ & $M^{2} / g^{2}$ \\
\hline 0 & 0 \\
1 & 5.9 \\
2 & 14.3 \\
3 & 23.4 \\
4 & 32.7 \\
\hline
\end{tabular}

This $q \tilde{q}$ spectrum, augmented by clusters of nearly equal-mass states by the additior of massless, low-monentum, mesons, provides a fairly accurate characteriza. tion of the full strong-coupling spectrum. 


\subsection{HADRON MASSES AT SMALL QLIARK MASS}

In the limit of vanishing quark mass, a great deal of information about the de. perdence of the meson and baryon masses as a function of the quark mass can be extracted from: the small- $x$ behavior of their wavefunctions. Consider first the me. son wavefunction $\phi(x)$ in the valence, or equivalently, the large- $\Lambda$ approximation, which obeys the eigenvalue equation

$$
M^{2} \phi(x)=m^{2}\left(\frac{1}{x}+\frac{1}{1-x}\right) \phi(x)-\frac{g_{N}^{2}}{\pi} \int_{0}^{1} d y \frac{\phi(y)-\phi(x)}{(y-x)^{2}} .
$$

The wavefunction $O(x)$ is normalized to one, and therefore

$$
M^{2}=m^{2} \int_{0}^{1} d x \frac{\phi^{2}(x)}{x(1-x)}-\frac{g^{2}}{\pi} \int_{0}^{1} d x \int_{0}^{1} d y \frac{\phi(x)[\phi(y)-\phi(x)]}{(y-x)^{2}} .
$$

The form of $\varphi(x)$ at small $x$ is understood, and this can be exploited by examining quantitjes which are sensitive to this region, as in Re[s. [22], [18; and [13]. A particularly useful trick is to express the dependence of $M^{2}$ on the quark mass $m^{2}$ in terms of the free Hamiltonian. By the Hellmann-Fe!nman theoren:.

$$
\partial_{m^{2}} M^{2}=\partial_{m^{2}}\left(M \Lambda^{2}\right\rangle=\left\{\partial_{m^{2}} M^{2}\right\}
$$

and since

$$
M^{2}=m^{2} H_{0}+g^{2} H_{1}
$$

then

$$
\partial_{m^{2}} \cdot 1 t^{2}=\left(H_{0}\right)
$$

The brackets here indicate the expectation value within the meson. This is esire- 
cially useful, as it relates $\partial_{m^{2}} M^{2}$ to the expectation value of a simple, diagonal operator. In the valence approximation,

$$
\partial_{m^{2}} M^{2}=\int_{0}^{1} d x \frac{\phi^{2}(x)}{x(1-x)} .
$$

Though generally true, it is useful in the $\mathrm{m} / \mathrm{g} \rightarrow 0 \mathrm{Jimit}$. For $x \rightarrow 0$ (or 1 ). $\phi(x)-c x^{a}$ (or $\left.c(1-x)^{a}\right)$, with $c$ independent of $x$ and equal in magnitude at both endpoints by parity: As $m \rightarrow 0, a \rightarrow(3 / \pi)^{1 / 2}\left(m / g_{N}\right)$. In this limit, $\phi(x)$ in Eq. (3.111) is approaching a constant at the endpoints and these regiots then dominate the integral. In this linmit

$$
\partial_{m^{2}} M^{2} \rightarrow \frac{c^{2}}{a}=c^{2}\left(\frac{\pi}{3}\right)^{\frac{1}{2}}\left(\frac{g_{N}}{m}\right)
$$

or, integrating.

$$
M^{2}=M_{(m=0)}^{2}+2 c^{2}\left(\frac{\pi}{3}\right)^{\frac{2}{2}} g_{N} m
$$

to leading order in $m$. The mass $M_{m=0}^{2}$ is independent of $m$. ard so must be proportional to $g^{2}$, if not zero. For lightest meson, $M_{(m=0)}^{z}$ is known to be zero. In the valence approximation, $\phi(x)$, and therefore $c$, approaches one as $m / g \rightarrow 0$. and therefore the leading term for the lightest meson is

$$
M^{2}=2\left(\frac{\pi}{3}\right)^{\frac{2}{2}} g x n
$$

Also. it might be noted tha: because in genera!

$$
m^{2}\left\langle H_{0}\right\rangle=c^{2}\left(\frac{\pi}{3}\right)^{\frac{1}{2}} g x m=\frac{1}{2} \cdot H^{2}
$$


then in this limit

$$
m^{2}\left\langle H_{0}\right\rangle=g^{2}\left\langle H_{I}\right\rangle=\frac{1}{2} M^{2}
$$

The same argument may be advanced for $U\left(N^{*}\right)$. except that $g_{\Lambda}^{2}$ is replaced by $g(N / 2)^{1 / 2}$. For the lightest meson, $M_{(m=0)}^{2}=g^{2} / 2 \pi$, and $c=1$. This is true even without restricting to the valence wavefunction, as this occurs dynamically: Then. for $L(A)$

$$
M^{2}=\frac{g^{2}}{2 \pi}+2\left(\frac{\pi}{3}\right)^{\frac{2}{3}}\left(\frac{N}{2}\right)^{\frac{1}{2}} g m+\cdots
$$

This discussion applies to the $S L^{\prime}(\Lambda)$ baryon as well as to the meson as $\mathrm{m} / \mathrm{g} \rightarrow$ 0 . since the wavefunction behaves in the same way as each argument $x_{1} \sim 0$. Ful each $x_{1}$ there is a term $m^{2} / x_{1}$ in $H_{0}$ which picks it out in the region near zeto. As a result,

$$
M_{B}^{2}=M_{B_{1 m=0 j}}^{2}+2 c_{B}^{2}\left(\frac{\pi}{3}\right)^{\frac{1}{2}}\left(\frac{1}{2}\right)^{\frac{2}{2}} g m+\ldots
$$

for valence $B=1$ states. The coefficient $c_{B}^{\prime}$ here is given by the intc:a!

$$
\int d x_{2} \cdots d x_{N} \varepsilon\left(1-\sum_{1 \neq 1} x_{1}\right) \bar{\phi}_{B}^{2} ! x_{2} \cdots x_{1} 1
$$

where

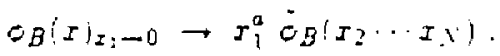

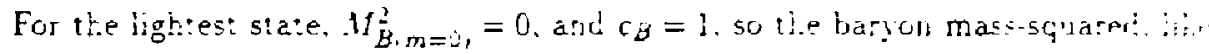

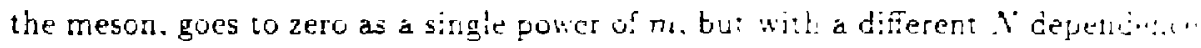


Because this discussion relies only on the small- $x$ behavior of the wavefunctions. and because $H_{0}$ is diagonal, it is simple to extend it to the case where higher-Fock states are included. Each $n$-particle state contributes a term $2 c_{n}^{2}\left(\frac{\pi}{3}\right)^{\frac{1}{2}}\left(\frac{x}{2}\right) g . n m$, with $c_{n}$ defined as for the baryon. A great deal of inieresting physics for finite $N$ is tucked away into the coefficients $c_{n}$, which may at least be extracted numericalis. although with some effort, and possibly analytically.

\subsection{COMPARISON WITH RESLLTS FROM BOSONIZATION}

As has been seen. the dependence of the mass of the lightest meson and bay.u! states on the quark mass and coupling in the limit $m / g \rightarrow 0$ mas be related tu $i$,.. asymptotic small-x beilavior of wavefunctions. This bctavior is shared by bo:h the valence and higher-Fock states of mesons and baryons. lowest-lying and excited. As a result, all barion masses obey

$$
\partial_{m^{2}} M^{2}=\left[\sum_{n}(n / 2) c_{n}^{2}\right]\left(\frac{\pi}{3}\right)^{\frac{1}{2}} \frac{g N}{m}
$$

where $n$ runs over the particle number of each wavefunction. That is, $n=2.4 .6 \ldots$

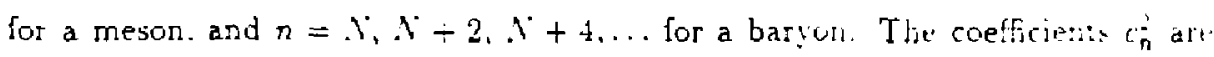
defined as in the previous section.

In the valence or large- $\hat{\lambda}$ approximation, only the leading $c_{n}^{\prime 2}$ is retainet. Whict. for the lowest-lyitig meson or baryon, appraaches one as $m / g \rightarrow 0$. The result fin

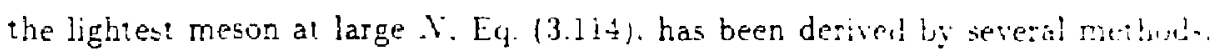
beginaing with 't Hooft. ${ }^{21,26 ;}$

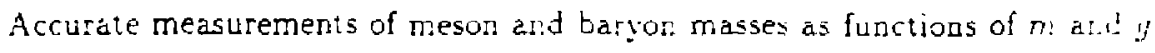

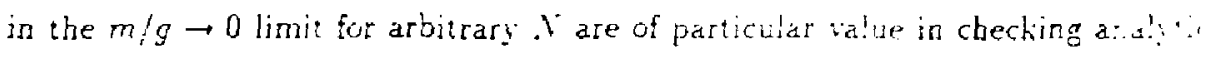


results obtained by several authors by means of bosonization. ${ }^{[4+4 s]}$ These yield a mass for both the lightest baryon and meson (for one flavor) which goes to zer, with $m$ as

$$
M^{2} \propto\left[m^{2}\right]^{\frac{1}{2}+\varepsilon}\left[g^{2}\right]^{\frac{1}{2}-\varepsilon}
$$

with

$$
\delta=\frac{1}{2(2 N-1)} .
$$

Clearly in the large $A$ limit, $\delta$ vanjshes, consistent with the results above. However. fur $A$ finite. 6 can be checked numerical!:

The valence approximation produces the large $-\Lambda^{\prime}$ result $\delta=0$ for all hadrons: therefore, non-zero $\delta$ must be due to the presence of bigher-Fock states. Furthermore. Eq. (3.120) depends only on the universal small- $x$ behavior of wavefunctions and is true in general. To produce a relation such as Eq. (3.121) upon integrating Eq. (3.120), the coefficients $c_{n}$. which are independeni of $x$. must hoiveret derent. on $m / g$ according to

$$
c_{n}^{2} \times(m / g)^{8}
$$

that is. as $x \rightarrow 0$. the leading dependence of $\rho(x)$ on both $x$ and $m / g$ must be such that

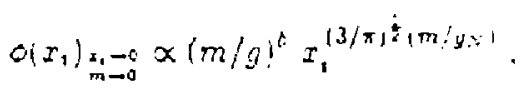

where only the leading behavior of the power of $x_{1}$ is retained. The suppresitit. by the factor $(m / g)^{\varepsilon}$ for smali $m$ is extrenely mild. and is not significant uix., $\mathrm{m} / \mathrm{g} \sim \epsilon^{-1 / \varepsilon}$. If $\varepsilon$ is given $\mathrm{by} \mathrm{Eq}_{\mathrm{q}}\left(3.121 !\right.$ theri $\epsilon^{-1 / t}=\epsilon^{-2 \cdot 2 \cdot-1)}$ which dectrit... rapidly with $M$ going from $2 \times 10^{-1}$ a: $A=2$. to $0 \times 10^{-5}$ at $N=4$. 
Certain qualitative features should be observed in the wavefunctions if $\delta$ is given by Eq. (3.121). First, including higher-Fock states should suppress the valence wavefunction $\phi_{U}(x)$ near $x \sim 0, m \sim 0$ by roughly a factor of $(\mathrm{m} / \mathrm{g})^{6}$ relative to the valence approximation. In Table [10], the decrease of the $q \bar{q}$ wavefunction for che $N=2$ meson at $2 K=24$, after including $q \bar{q} q \bar{q}$ higher-Fock states, is given for several $\mathrm{m} / \mathrm{g}$ and $\boldsymbol{x}$ in this region, qualitatively confirming this. For $N=2$. Eq. (3.121) gives $\delta=1 / 6$.

Table $[10$

\begin{tabular}{|cccc|}
\hline$m / g(m / g)^{\delta=1 / 6}$ & $x$ & $\phi_{2}(x) / \phi_{2}(x)_{\text {ral appr }}$ \\
\hline .4 & .86 & $5 / 24$ & .98 \\
& & $3 / 24$ & .96 \\
& & $1 / 24$ & .91 \\
.2 & .76 & $5 / 24$ & .99 \\
& & $3 / 24$ & .95 \\
& $1 / 24$ & .89 \\
\hline
\end{tabular}

Second. this suppression and the importance of bigher-Foch siates stould diminish for larger $N$. As is evident in Fig. (19b), the magnitude of the qgis $\bar{q}$ higher-Fock state decreases b; two orders of magnitude from $\mathrm{X}=2$ i0 4 . The cor: current loss of probability from the ends of the valence wavefunction diminist.es: comparatly:

The exponent 3 for the general case $2 f^{2} \times \mathrm{m}^{3}$ as $n \rightarrow 0$ can be extracied Dumerically by measuring $\beta=M^{-2} m \partial_{m} .11^{2}$. Attempts at this have prosi.c(5.! results which. although consistent have large ersurn au! a realistic check w. 3. 
while worthwhile. will require much larger values of $h$.

Another general bosonization result for the lightest meson and baryon masses as $m / g \rightarrow 0$ ? $^{103}$

$$
M_{\text {meson }} / M_{\text {baryon }}=2 \sin \left[\frac{\pi}{2\left(2 . N^{+}-1\right)}\right] \text {. }
$$

has been cheched with slightly greater success. Ia particular. $M_{\text {mes }} / M_{b a r}$ was computed for $\lambda$ from 2 to 4 at several values of $2 K$ up 10 about 20 and thes: extrapolated. Computing the ratjo at each $k$ prior to extrapolation proved more accurate than extrapolating the baryon and meson masses independent?y.

Table [11]

\begin{tabular}{|ccc|}
$N$ & $2 \sin \left(\frac{\pi}{21 . I^{2}-1 !}\right)$ & $2 / \lambda$ \\
\hline 2 & 1 & 1 \\
3 & .6150 & .5667 \\
4 & .4450 & .5 \\
\hline
\end{tabular}

For $N=2$, the ratio is exactly one at all $K$ and $\mathrm{m} / \mathrm{g}$. while the results for $X=3$ and 4 are presented in Table [12]. These are clearly consisinat, athoug!. with large errors, with those of Eq. (3.129) (Table [11]) approaching it from the nonrelativistic value of $2 / N$ obtained by adding quart masses. The numbers in parentheses are the uncertainties in the firal digit, estimated by the last teli:: retained in the extrapolation series. 
Table [12]

\begin{tabular}{|c|cc|}
\hline & $M_{\text {mes }} / M_{\text {bar }}$ \\
$\mathrm{m} / \mathrm{g}$ & $N=3$ & $N=4$ \\
\hline$\propto$ & $2 / 3$ & $1 / 2$ \\
1.6 & $.656(5)$ & $.48(1)$ \\
.8 & $.647(3)$ & $.477(6)$ \\
.4 & $.639(7)$ & $.468(3)$ \\
.2 & $.64(7)$ & $.46(2)$ \\
.1 & $.63(4)$ & $.46(4)$ \\
.05 & $.62(5)$ & $.46(4)$ \\
\hline
\end{tabular}

\subsection{MATRIX ELEMENTS FOR SMALL QLARI MASS}

Once baving computed the full set of wavefunctions, not only masses but an: other quantity desired (and well-defined) can be obtained simply by computing integrals. In this section, several analytic results for matrix elements, mainly: vacuum-to-one-meson, will be presented, specifically for small quark mass, as an illustration.

The small-mass limit is of particular interest because several exact (main:? large- $\mathrm{N}$ ) results become accessible. while numerical accuracy becomes increasing: difficult. Furthermore, certain anxieties about this region potenially provaked previous discussions may be addressed First, for finite quark mass. bunndary terns: are neglected. and

$$
\left.\left.t_{L}\left(0 . x^{-}\right)=-\frac{1 m}{4} \int_{-L}^{L} d y^{-} t\left(x^{-}-y^{-}\right) x^{\prime}\right) \cdot y^{-}\right) .
$$


Only $\psi_{R}$ is treated as a dynamical degree of freedom, and for vazishing quark mass. $\psi_{L}$ apparently decouples. leading to an unacceptable loss of degrees of freedum. Second, mesons and baryons become massless simultaneously, giving conflicting suggestions about chiral symmetry.

U'sing the continuum forms for the fields, wavefunctions, and commutation relations, the matrix elements

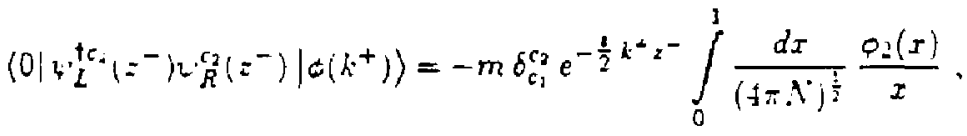

$$
\begin{aligned}
& \left\langle 0\left|L_{R}^{+c_{2}}\left(z^{-}\right) L_{L}^{c_{2}}\left(z^{-}\right)\right| \varphi\left(k^{+}\right)\right\rangle=m \delta_{c_{1}}^{c_{2}} e^{-\frac{1}{2} k^{+} z^{-}} \int_{0}^{1} \frac{d x}{(4 \pi N)^{\frac{1}{2}}} \frac{\phi_{2}(x)}{1-x},
\end{aligned}
$$

and

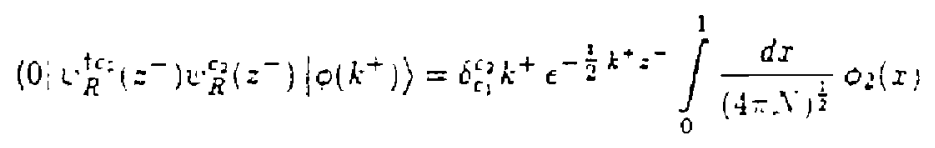

Directly from these, the scalar, pseudoscalar, and gauge-curredt matrix elerinenis are

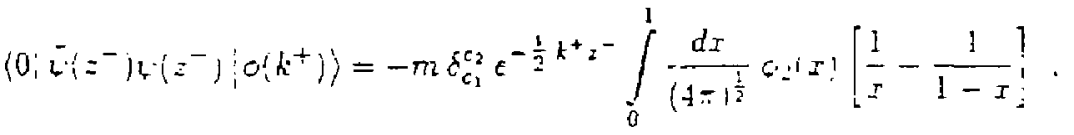

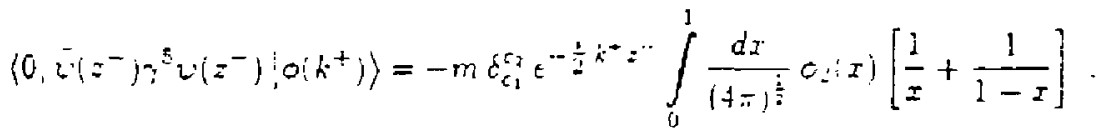


and

$$
\left\langle 0\left|j^{+0}\left(z^{-}\right)\right| \phi\left(k^{+}\right)\right\rangle=2 \operatorname{Tr}\left[T^{a}\right] k^{+} e^{-\frac{i}{2} k^{+} x^{-}} \int_{0}^{1} \frac{d x}{\left.(4 \pi)^{2}\right)^{\frac{3}{2}}} d z(x) .
$$

The last vanishes, except for the $U(1)$ current for which $\operatorname{Tr}\left[T^{0}\right]=\left(N^{\prime} / 2\right)^{1 / 2}$. In accordance with the discussion on parity, only pseudascalar states ( $\phi_{2}$ odd under $x \mapsto 1-x$ ) couple to $\bar{\psi} \gamma^{5} \psi$, and scalar states (even under $x \mapsto I-x$ ) to $\bar{\psi} \psi^{\prime}$, as would be expected. ${ }^{[2]}$

$\nvdash: L$ js proportional to the quark mass $m$, and is responsible for the $m$ in front of Eqs. (3.129) and (3.130). These matrix elements appear to vanish with $m$. and were this the case, it would be an unwelcome symptom that $t_{L}$ decoupies. However. as discussed previously, as $m \rightarrow 0$, the integrals over $0_{2}(x)$ are dominated by the endpoints. where $0_{2}(x)_{x \rightarrow 0} \sim c x^{a}$, and similarly for $1-x \sim 0$.

A factor of $1 / m$ from the integral exactly cancels the $m$ from $i L$. The result is a weil-defined limil.

$$
\left\langle 0\left|L^{-}\left(z^{-}\right) h^{5} \psi\left(z^{-}\right)\right| \phi\left(k^{+}\right)\right\rangle \rightarrow c(N / 3)^{\frac{1}{4}} g \varepsilon^{-\frac{1}{2} k^{+} z^{-}}
$$

when $\left|\sigma\left(k^{+}\right)\right\rangle$is a pseudoscalar, with the same result for $i^{-}{ }^{\prime}$ and a scalar state. Apparenty: the asymmetric treatment of $\iota_{L}$ and $\tau_{R}$ in quantization is exarly compensated for dynamically. In particular, the singlar $1 / p^{-}$in the expansion fut $\psi_{L}$ preverts it from vanishing with $m$ in matrix elements.

This provides fairly convincirg evidence that the standard light-cone quar:ization is adequate for finite quark rass. and thai the limit $m \rightarrow 0$ is se.tifi. 
but also that for $m=0$ identically, special care is required during quantization to retain $\psi_{L}$.

The singular $m / p^{+}$in $\psi_{L}$, as in Eq. (1.105), and the corresponding kinetic term $\mathrm{m}^{2} / x$ in the Harniltonian, while potentially ticklish, have actually proven advantageous in extracting the small- $x$ behavior of wavefunctions and understanding the theory for small quark mass. Also, as discussed in Ref. [18], the leading behavior for the large- $N$ meson form factor is also governed by this region of the wavefunction. Numerical and analytic results for form factors at finite $N$, including counting rules at large $Q^{2}$, will be presented in subsequent work.

The behavior of the lightest meson as the quark mass vanishes is of particular interest and has been studjed in the large- $N$ limit extensively: ${ }^{[36.22 .18 .39 .06 .03]}$ It has already been noted that this particle is a pseudoscalat whose mass squared goes to zero uith the quark mass as

$$
M^{2} \sim 2 c^{2}\left(\frac{\pi}{3}\right)^{\frac{1}{2}} g_{N} m
$$

with $c \sim 1$ in the large- $N$ or valence approximation. This is all suspiciously suggestive of a two-dimensional pion; that is, the Goldstone boson associated with the' spontaneous breakdown of chiral symmetry. Furthermore. fron Eq. (3.11i)and Fig. (10), if $\lambda$ is taken to infinity before $m$ is allowed to vanish. the mass of the lowest-lying baryon is infinite, another indication of symmetry breaking. A: frequently pointed out. [..35. .5$]^{3}$ the order of limits is important. Finally. repeating the standard derivation for the chiral condensate $\langle 2 \cdot\rangle\rangle$. beginning with a piun interpolating field $\phi_{\pi}$ from the divergence of the $[(1)$ axia! current

$$
\partial_{\mu} j_{5}^{\mu} \equiv \sqrt{2} f_{\pi} m_{z}^{2} o_{\pi}
$$


and using Eq. (3.132) with $c=1$ yields ${ }^{[(3)}$

$$
f_{\pi}=(N / 2 \pi)^{\frac{1}{2}}
$$

and

$$
\langle 0|: \bar{\psi} \psi:| 0\rangle=-\frac{N}{(12 \pi)^{\frac{1}{2}}} 4 N
$$

This is very curious. By the nature of the light-cone formalism, no non-trivial vacuum behavior is permitted with any non-zero quark mass. Nevertheless, formal arguments in the large- $N$ limit which depend only on the nature of the pseudoscalar meson wavefunction at small $x$ yieid a non-zero (in fact, infinite for $N \rightarrow \infty$ ) value for $\left\langle\bar{w} \imath^{6}\right\rangle$ as $m$ vanishes. ${ }^{[+7]}$

This seems to directly conflict with Coleman's theorem ${ }^{(f)}$ that Goldstone bosons cannot exist in two dimensions, due to the severe infrared divergences from the ill-defined delta function $\delta\left(k^{2}\right)=\delta\left(k^{+} k^{-}\right)$associated with massless scalars. An allowed exception in Ref. [i] is one which decouples, such as a scalar constructed from a pair of non-interacting massless fermions.

The scalar $\bar{\psi} \psi$, for example. does have zero probability to create such a pair: that is (ignoring color)

$$
\left\langle 0\left|\bar{q} \cdot(z) b_{(1-x) p^{+}}^{\dagger} d_{x p^{+}}^{\dagger}\right| 0\right\rangle=m \epsilon^{-i p \cdot z}\left[\left(\frac{x}{1-x}\right)^{\frac{1}{2}}-\left(\frac{1-x}{x}\right)^{\frac{1}{2}}\right]
$$

which vanishes as $m \rightarrow 0$. However, the Jarge- $\Lambda$ pseudascalar does not decoupice in this manner, as evidenced by Eq. (3.132) (with $c=1$ ). On the other hatri. the operators which create this pion have been shown to satisfy free commutatis: 
relations, and they commute with the operators in the Hamiltonian. Finally, in at least one case where a term which couples to these operators is added to $P_{I}$ by introducing an extra $U(1)$ inleraction, these pions acquire a mass, $g /(2 \pi)^{1 / 2}$, obviating this dilenma.

As a final curiosity which illustrates the importance of the order of limits, note that for $U(N)$, the $U(1)$ chiral anomaly produces an additional contribution of $g^{2} /(\delta \pi m)$ for $\langle\bar{\psi} \psi\rangle$. In the large- $N$ limit this vanishes, while for finite $N$, it diverges as $m \rightarrow 0$.

For finite values of $\Lambda$, the situation is somewhat clearer. When $m \rightarrow 0$, the lightest baryon mass also vanishes. Also, acting on existing states with the operator $V_{k}^{0}$, which creates the massless pseudoscalar meson, can flip parity without altering mass if $k$ is zero. As a result, parity doublets are trivial to generate. These would seem to indicate that chiral symmetry is restored as the quark mass vanishes.

Furthermore, bosonization results imply that the asymptotic coefficients $c$ which appears in. for example Eqs. (3.132) and (3.133), vanish slowly with quark mass as $(\mathrm{m} / \mathrm{g})^{6}$, with $\delta=1 /[2(2 N-1)]$. Numerical results have been presented which indicate that this is likely the case. As a consequence, ever though the meson mass still vanishes, it vanishes proportionally with $m^{1+\delta}$ rather than $m$. If this is correct, operators such as $\bar{\psi}^{5} \psi^{5}$ do indeed have zero probability of creating a massless meson from the vacuum, although the probability vanishes exirentely slowly in $m$ for any sizable $A$. 
CONCLUSIONS

In this work, $Q C D$ in $1+1$ dimensions was quantized on the light-cone, and the role of boundary terms clarified. By imposing antiperiodic boundary conditions in $\boldsymbol{x}^{-}$, a discretized Hamiltonjan was constructed and diagonalized for several gumbers of color $N$ and one quark flavor. At relatively little cost in computer time, the full spectra and wavefunctions were produced, and these were in agreement with results from otber calculations when available. Finally, the nature of solutions in the limits of both strong and weak coupling was discussed.

Even in tro dimensions. there is still a greal deal that might be done to exterd these results. By studying systems with higher baryon number, the nucleas potential that results from the linear interquark potential could be determined. Form factors iv: baryons and higher-Fock states could be computed and counting rules at large momentum transfer derived. Finally, the inclusion of several flavors would bring the model closer $t$ to reality. It would allow for a more general study of chiral symmetry and for the calculation of such things as the strangeness content of the proton, at least in two dimensions.

Of course, physics is conducted in (at least) four dimensions, where this numerical quantization scheme may or may not provide a successful approach to QCD. It was. however, the way to solve it in two dimensions, and there is as yet no reasuli to be discouraged. 


\section{APPENDIX A \\ MATRIX ELEMENTS AND COLOR CONTRACTIONS}

A typical matrix element of creation and annibilation operators which must be computed both for pormalization and for evaluating the Hamitonian is (for $\Lambda=3$ )

$$
\begin{aligned}
& \epsilon_{c_{1} \varepsilon_{2} c_{3}} \epsilon^{a_{1} a_{2} a_{3}} \delta_{c_{1}}^{c_{3}} \varepsilon_{a_{3}}^{a_{4}} f(k, \ell) \times \\
& \quad\left\langle 0\left|b_{\varepsilon_{1}, a_{3}} b_{\ell_{2}, a_{2}} b_{\ell_{3}, a_{3}} b_{\ell_{1}, a_{4}} d_{\ell_{3}}^{a_{3}} d_{k_{3}, c_{3}}^{\dagger} b_{k_{1}}^{t c_{3}} b_{k_{3}}^{\dagger c_{3}} b_{k_{2}}^{\dagger c_{2}} b_{k_{3}}^{\dagger c_{1}}\right| 0\right\rangle,
\end{aligned}
$$

where $c_{1}$ and $a_{1}$ are color indices, $k_{1}$ and $c_{1}$ are momenta, with $f$ some function of these such as the photun propagator. Baryonic and mesonic singlets are formed by contraction of color jndices with epsilon and delta tensors. In all matrix ele. ments color indices will be completely contracted, since the Hamiltonian as well as the Fock sijace are color singlets. A small amount of reflection indicates (and many minutes of wasted CPU confirm) that it would be advantageous to compuie the contribution from contracting color indices diagrammatically rather than it. eratively. especially for large $\Lambda$. That is, to follow the flow of color through the epsilon and delia tensors. count closed loops, and assign factors of $\Lambda^{*}$ rather than assign speciñc numbers to color indices and then sum.

The operators which carry color indjces also depend on monentum. Beruit corrputing color factors, particular values for moment a are assigned. and the var. uum expectation of these operators converted into products of delta functiors in color. In this appendix, the algorithm used by the program to evaluate genciai matrix elements and color sums is presented. 


\section{A.1. A SIMPLE ALGORITHM FOR THE EVALLATION OF CREATION AND ANNIHILATION MATRIX ELEMENTS}

Matrix elements such as

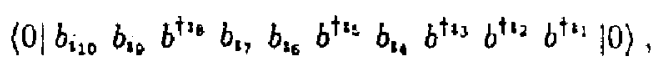

where $i_{1}$ to $i_{10}$ may incorporate a set of indices such as color, momenturn. or spin, may be computed using Wick's theorem. A recipe which is more efficient and compact for both mechanical and human computers takes advantage of the symmetries due to the anticommutation of the operators to generate all the possible contractions with appropriate signs from one initial product of delta functions. To compute an element such as $E q .(A .2)$ :

\section{Construction of Initial Term}

Proceeding from right to left. skip creation operators and stop at the firs: annibilation operator. Pair this with the creation opeator to its immediate riglat io form a delta ( $\delta_{1,}^{t_{y}}$ in this case) and remove these from the matrix element. Continue left, pairing and removing operators until only the product

$$
\delta_{1_{4}}^{13} \delta_{1 c}^{1:} \varepsilon_{i 7}^{12} \delta_{19}^{25} \delta_{t_{10}}^{11}
$$

remains. The overall sign of this term is positive since no operators were permuted. 


\section{Construction of Permutation Operators}

Beg.nning again with the matrix element Eq. (A.2), proceed left until hitting the first annihilation operator. The first permutation operator

$$
1-P_{3,2}-P_{3,1},
$$

which will perntute indices in Eq. ( $\therefore .3$ ), is built by subtracting from 1 operators which independently swap the index of the creation operator to the immediate right of the cursent annihilation operator with each subsequent creation operatur index to its right. Remove from Eq. ( $\therefore 2)$ this annihilation operator and the creation operator to its immediate right and proceed left to the next annihilation oresator, repeating the procedure. The next permutation is

$$
1-P_{5,2}-P_{5,1}
$$

This second operator is placed to the right of operator Eq (A.t). Con:jnur left until all annihilation operators are exhausted. The final result is

$$
\left[1-P_{3,2}-P_{3,1}\right]\left[1-P_{5,2}-P_{5,1}\right]\left[1-P_{2,1} !\left[1-P_{3,1}^{\prime}\right]\right.
$$

Which then acts upon the indices of the initiat term Eq. (A.3). giving the 36 terms with appropriate signs:

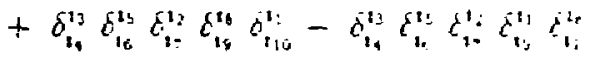

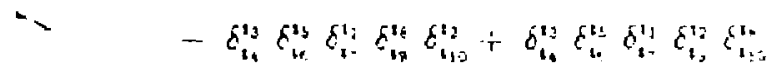

$$
\begin{aligned}
& +\cdots
\end{aligned}
$$

One of the advantages of this procedure is that all the infortnation is contaitien! 
compactly in the operator Eq. (A.5) acting on Eq. 1.3) with the symmetries manife . In cases, for example, when operators are contracted into epsilon tensors, many of the permutations are clearly redundant and may be converted into numerical factors.

Finally, s.zatrix elements in general will include creation operators and annihilation operators which cannot be contracted with one another, as for example. antiquark versus quark, or operators where some types of indices such as flavor are fixed and different. In these cases, an overall sign is computed by determining the operator interchanges needed to construct an initial term such as in Eq. (A.3).

\section{A.2. COLOR SLMS}

The vacuum expectation value of creation and annililation operators has been converted into a sum of products of kronicker deltas in color indices. In each term these deltas are entirely contracted with the delta and epsilon tensors used to form the crlor singlet Fock states, and the deltas which appear contracted with the operators in the Hamiltonian, of the form $\delta_{c_{2}}^{c_{1}} \delta_{c_{4}}^{c_{3}}-(1 / N) \delta_{c_{1}}^{c_{1}} \varepsilon_{c_{2}}^{c_{3}}$ in $H_{I}$ and $\delta_{c_{2}}^{c_{1}}$ in $H_{0}$.

Computing the color factor has now been reduced to contracting a produrt of an arbitrary number of epsilon and delta tensors. For the case with only deltas. it is necessary only to associate a factor of $N$ with each closed color loop. Inops art formed by products of delias such as $\delta_{c_{3}}^{c_{1}} \bar{\varepsilon}_{c_{1}}^{c_{2}} \varepsilon_{c_{2}}^{c_{2}}$.

This is implemented in the program by constructing a vector $V^{\prime}(n)$ where tan.: 
$\delta_{c_{m}}^{c_{n}}$ is represented by an $m$ in the $n^{\text {th }}$ location. A closed loop is traced by muving from location $n$ to $V(n)=m$ and so on until finally returning to $n$.

For contractions which include epsilons, any deltas present are first traced through and eliminated. The fully contracted products of epsilons which remain are then converted into a sum of products of deltas by the identity (for $\lambda$ colors)

$$
\epsilon_{c_{1} c_{2} \cdots c_{N}} \epsilon^{c_{1} b_{2} \cdots b_{N}}=\delta_{c_{2}}^{b_{2}} \delta_{c_{3}}^{b_{3}} \cdots \delta_{c_{N}}^{b_{N}}-\delta_{c_{2}}^{b_{3}} \delta_{c_{3}}^{b_{2}} \cdots \delta_{c_{N}}^{b_{N}}+\ldots
$$

where the elipses include permutations of the $b_{4}$ with appropriate signs. The lit. sulting deltas are then contracted as betore. For a generai discussion on coniputing color factors diagrammatica!ly, see Ref. [48]. 


\section{APPENDIX B}

\section{EVALLATION OF INTEGRAL IN SECTION 3.3}

This appondix presents a calculation of the integial

$$
f_{-1}^{\infty} d u \frac{(1+u)^{a}-1}{u^{2}}
$$

which appears in $E_{q}(3.21)$.

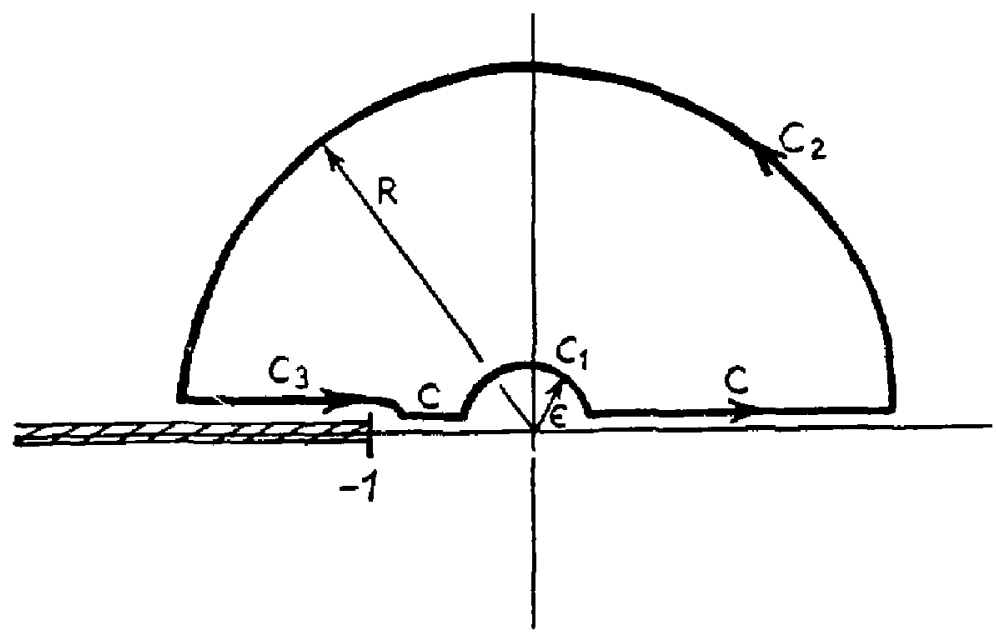

Figure 32. Integra.jon Contour

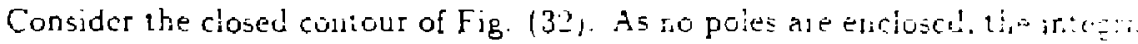
along contours $C_{1}, C_{2}$ and $C_{3}$ plus the desired integial along $C$ sun to ze...

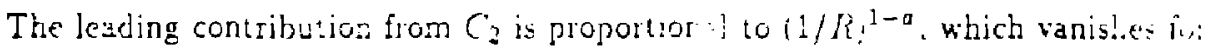

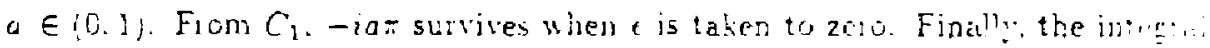


along $C_{3}$ is

$$
\int_{C_{3}} d u \frac{(1+u)^{a}-1}{u^{2}}=\int_{0}^{\infty} d t \frac{t^{2} e^{12 \pi}-1}{(1+t)^{2}}
$$

The second term in the integrand contributes -1 , while, by defiring $s=1 /(1+t)$. the first term may be rewritten as

$$
e^{i a \pi} \int_{0}^{1} d s s^{-a}(1-s)^{a}
$$

By the I iunction relacions ${ }^{i 4:}$

$$
\int_{0}^{1} d x x^{\alpha}(1-x)^{\beta}=\frac{\Gamma(1+\alpha) \Gamma(1+\beta)}{\Gamma(2+\alpha+\beta)}
$$

and

$$
\Gamma(z) \Gamma(1-z)=\pi \csc (\pi z) \text {. }
$$

the total for contour $C_{3}$ is

$$
-i 1+e^{1 a x} a-\csc i a-i .
$$

Sext. the same analysis is applied to the closed contour whitis reacots tle. contou in Fig. (32) across the real axis. Adding the two the cantibution front $C_{3}$ is cancelled. the refection of $C_{3}$ gives

$$
-1-\epsilon^{-t \omega \pi} a-\csc i=i
$$

and the frial result by Cauchy's theorem is

$$
\left.\int_{-1}^{x} d u \frac{(1+u)^{a}-1}{u^{2}}=1-a-c u: i r\right) \quad \text { E }
$$




\section{REFERENCES}

1. This introduction is based on material appearing in Hornbostel, K., Brodsky. S. J., and Pauli, H. C.,Procetdings of the Ohio State Horkshop on Relativistic Many-Body Physics, World Scientific, 1988.

2. Pauli, H. C., and Brodsky, S. J., Phy's. Rev. D32. 1993 (1985); D32, 2001 (1985).

3. For an introduction to light-cone quantization, cf. Jan. T.-M., Phys. Rev. D7, $1780(19: 2)$ and references therein.

4. Weinberg, S., Phys. Rev. 15C, 1313 (1966).

5. This is not strictly true :r there are quanta for which $p^{+}$is zero. See for example Harindranath, A., and Vary, J., Pbys. Rev. D37, 3010 (1988). There are other subtleties which will be addressed in future work.

6. Pauli. H. C.. and Brodsky, S. J.. Phys. Rev, D32. 1993, D32. 2001 l19s:.

i. Coleman. S., Comm. Matb. Phỵs., 31, $259(1073)$.

8. Bassetto, A., Lazzizzera, I., Soldati, R., Nucl. Phys. B236, 319 (1984).

9. Hälpern, M. B., and Senjanovic, P., Phy's. Rev. D15. 3629 (197i).

10. Bars, 1.. and Green. M. B., Ph:5. Rev. D17, 537 (1975).

11. McCartor. G., Mission Research Corp. Preprint, 1958. and private comau. nications.

12. Mathews, J., and Haller. R. L., Mathematzcal Mcthods of Physics. Benjamin/Cummings, $\mathrm{X} Y, 1970$. 
13. Rohrlich, F., Acta Phy's. Austr. 32, 87 (1970).

14. Steinhardt, P., Ann. Phys., 128, 425 (1980).

15. I would like to thank A. Tang for discussions on this point.

16. Kogut, J., and Susskind, L., Phys. Rev. D10, 3468 (1974).

17. Coleman, S., Jackiw, R., and Susskind, L., Ann. Phys. (N.Y.) 93, 267 (1975): Coleman, S., Ann. Pbys. (N.Y.) 101, 239 (1976).

18. Einhorn, M. B., Phys. Rev. D14, 3451 (1976).

19. See Ref. [41], ar $J$ also the comment by Coleman referenced in Ref. [10]. In discussions involving $U(N)$ which follow, the effect of this background field will be ignored.

20. Eller, T., Pauli, H. C., and Brodsky, S. J., Phys. Rev. D35, 1493 (1951). The notation of this paper will be followed as much as possible in this section.

21. 't Hooft, G., Nucl. Phys. B75, 461 (1974).

22. Callan, C. G., Coote, N., and Gross, D. J., Phỵs Rev. D13. 1619 (1976).

23. Bergknoff, H., Nucl. Phys. B122, 215 (197i).

24. For an excellent review of wavefunctions in QCD. including a discusison of normalizations, cf. Lepage, G. P., Bradsky, S. J., Huang. T., and Mackenzie. P. B., CLNS-82/522, published in Banff Summer lnstitute 81 (1981): also. Lepage, G. P., Brodsky, S. J., Phys. Rey. D22, $215 i$ (1980).

25. Bardeen, W. A., and Zumino, B., Nucl. Phys. B244, 421 (1984).

26. See for example, Goddard, P. and Olive. D., Int. J. Mod. Pbys. A1. 303 (1956). 
27. Press, W. H., Flannery, B. P., Teukalsky, S. A., Vetterling, W. T., Numerical Recipes, Cambridge Unjv. Press, Cambridge, 1986.

28. Hamer, C. J., Nucl. Phys. B195, 503 (1982).

29. Witten, E., Nucl. Phys. B160, 57 (1979).

30. Coleman, S., in Pointlike Structures Inside and Outside Hadrons, Proceedings of the 1979 Intl. School of Subnuclear Physics, A. Zichiclii, ed. Plenum Press. N.Y, $19 s^{\circ}$.

31. Blankenbecler, R., private communication.

32. Eller, T., thesis; unpublished.

33. Durgut, M., Nucl. Phys. B116, 233 (1976).

34. Bars, I, Nucl. Phys. B111, 413 (1970).

35. Webter. B. R. Nucl. Phys. B153, $45.5(1979)$.

36. Ref. [21]: see also, 't Hooft, G., in New Phenomena in Subruclear Physics. Proceedings of the 1975 Inti. School of Subnuclear Physics, A. Zichichi, ed. Plenum Press, N.Y.. 197T.

37. Hamer. C. J.. Nucl. Phys. B121, 159 (1977), B132, 542 (1975).

3E. The massless state obtained by acting once on the vacuum wih $l_{k}^{a}$ ha: heel: discussed in Buchmuller, W., Love. S. T., and Peccei, R. D.. Phys. Lett. 108B. 406 (1982\}.

39. Frishman. Y., Nuc\}. Plyys. B148. it $\{1979)$. 
40. This is the group considered by 't Hooft in Ref. [21]: the two are equivalent in the large- $N$ limit. In general the extra $C^{t}(1)$ introduced need not be associated with the same coupling constant as $S L^{\top}\left(N^{r}\right)$, but this is sufficient for this discussion

41. Coleman, S., Jackiw, R., and Susskind, L., Ann. Phys. (A.Y.) 93, 267 (1975); Coleman, S., Ann. Phys. (N.Y.) 101, 239 (19i6).

42. Witten, E. Comm. Math. Phys. 92, 455 (19S4).

43. Zhitnitskii. A. P., Sov. J. Nucl. Phys. 43, 999 (1956).

41. Steinhardt, P., Nucl. Phys. B176, 100 (1980).

45. Date. G. D., Frishman. Y., and Sonnenschein, J., Nucl. Phys. B253, 31,; (198i), and Sonnenschein. J., private communicaticn.

46. Amati, D., and Rabinovici, E. Phys. Lett. 101B, 407 (1981).

4i. A lattice calculation of this chiral condensate in this model is presented in Grasdou. T.. Cho, H. T., and Fried, H. M.. Phys. Rew D37. $910110: 8$.

15. Cvitanovic. P.. Group Theory. Nordita Notes. 195: Cvitanoric, P., Phyj. Rev. D14, 1530. (1976).

49. Abramowitz. M., and Stegun. I. A.. Hardjook of Mathematical Funciors Dover Púvications. XY. 1072. 